\title{
New vortex-string worldsheet theories from supersymmetric localization
}

\author{
Efrat Gerchkovitz and Avner Karasik \\ Department of Particle Physics and Astrophysics, Weizmann Institute of Science, \\ Rehovot 76100, Israel \\ E-mail: efrat.gerchkovitz@weizmann.ac.il, \\ avner.karasik@weizmann.ac.il
}

ABSTRACT: We use supersymmetric localization techniques to study the low-energy dynamics of BPS vortex-strings in four-dimensional $\mathcal{N}=2$ theories. We focus on theories with $\mathrm{SU}\left(N_{c}\right) \times \mathrm{U}(1)$ gauge group and $N_{f}$ hypermultiplets, all in the fundamental representation of $\mathrm{SU}\left(N_{c}\right)$ but with general $\mathrm{U}(1)$ charges. Recently, we proposed a condition that determines whether the low-energy string dynamics is captured by a two-dimensional worldsheet theory that decouples from the bulk [1]. For strings for which this decoupling applies, we propose a prescription for extracting the two-sphere partition function of the string worldsheet theory from the four-ellipsoid partition function of the parent theory. We obtain a general formula for the worldsheet two-sphere partition function in terms of the parameters of the four-dimensional theory and identify $\mathcal{N}=(2,2)$ GLSMs that possess these partition functions in a large class of examples. In these examples, the weak coupling regime of the four-dimensional theory is mapped to the weak coupling regime of the worldsheet theory. In addition, we study the classical string zero-modes in flat space and obtain predictions for the worldsheet spectra, which agree with the low-energy spectra of the GLSMs obtained in the localization analysis. For $N_{f}=2 N_{c}=4$, we discuss the map between string worldsheet theories under four-dimensional $S$-duality and use our prescription to study examples in which the weak coupling regime of the four-dimensional theory is mapped to the strong coupling regime of the worldsheet theory.

Keywords: Extended Supersymmetry, Nonperturbative Effects, Solitons Monopoles and Instantons, Supersymmetry and Duality

ARXIV EPrint: 1711.03561 


\section{Contents}

1 Introduction and summary 1

2 The classical string equations and the string moduli space 6

2.1 The classical vacuum and string equations 6

$\begin{array}{ll}2.2 & \text { The string moduli } \\ & 10\end{array}$

3 Examples of worldsheet theories 12

$\begin{array}{ll}3.1 \text { The equal charge case } & 12\end{array}$

$\begin{array}{lll}3.2 & \text { Strings with no } \tilde{q} \text { excitations } & 13\end{array}$

3.2.1 Proposal for the worldsheet theory 13

$\begin{array}{lll}3.2 .2 & \text { Comparison with the classical zero-mode analysis } & 14\end{array}$

$\begin{array}{lll}3.3 & \text { Strings with } q \text { and } \tilde{q} \text { excitations } & 16\end{array}$

$\begin{array}{lll}\text { 3.3.1 Proposal for the worldsheet theory } & 16\end{array}$

$\begin{array}{lll}\text { 3.3.2 Comparison with the classical zero-mode analysis } & 17\end{array}$

4 Two-sphere worldsheet partition functions from the four-ellipsoid partition function

$\begin{array}{ll}\text { 4.1 The four-ellipsoid partition function } & 18\end{array}$

4.2 Worldsheet sphere partition functions from the four-ellipsoid partition $\begin{array}{ll}\text { function in the equal charge case } & 20\end{array}$

5 General U(1) charges $\quad 27$

5.1 The worldsheet two-sphere partition function for a general string 27

$\begin{array}{lll}5.2 & \text { Identifications of worldsheet theories } & 31\end{array}$

$\begin{array}{lll}5.2 .1 & \text { Strings with no } \tilde{q} \text { excitations } & 31\end{array}$

5.2.2 Strings with $q$ and $\tilde{q}$ excitations 32

6 Strings in $N_{c}=2, N_{f}=4$ SQCD under triality 35

$\begin{array}{lll}6.1 & \mathrm{SO}(8) \text { symmetry and triality } & 35\end{array}$

6.2 Strings in $N_{c}=2, N_{f}=4$ SQCD under triality - localization analysis $\quad 39$

$\begin{array}{lll}6.2 .1 & \text { The } c_{i}=1 \text { case } & 39\end{array}$

6.2.2 T-dual of the $c_{i}=1$ case $\quad 40$

6.2.3 $S$-dual of the $c_{i}=1$ case $\quad 41$

6.2.4 Other transformations 45

A Non-perturbative contributions $\quad \mathbf{4 5}$

A.1 Non-perturbative contributions to the four-ellipsoid partition function $\quad 45$

A.2 Non-perturbative contributions to the two-sphere partition function 47

$\begin{array}{ll}\text { B Useful identities } & 48\end{array}$

$\begin{array}{ll}\text { C Derivation of equation (6.39) } & 49\end{array}$

$\begin{array}{ll}\text { D Consistency check } & 51\end{array}$ 


\section{Introduction and summary}

$2 \mathrm{~d}$ sigma-models have been long known to serve as useful toy models for $4 \mathrm{~d}$ gauge dynamics. The similarities between the $2 \mathrm{~d}$ and $4 \mathrm{~d}$ dynamics, in some cases, can be demonstrated not only at the qualitative, but also at the quantitative level. One example is the observed matching between the quantum BPS spectra of $2 \mathrm{~d} \mathcal{N}=(2,2)$ supersymmetric $\mathbb{C P}^{N-1}$ sigma model and $4 \mathrm{~d} \mathcal{N}=2 \mathrm{SU}(N)$ gauge theory with $N$ fundamental hypermultiplets [2]. This observation received a physical explanation in later works [3-6], which showed that by gauging a U(1) flavor symmetry in the $4 \mathrm{~d}$ theory mentioned above and introducing a FayetIliopoulos (FI) term, one obtains a theory that supports BPS vortex-strings. The string with the minimal winding number is part of a $\mathbb{C} P^{N-1}$ moduli space of string solutions. The low-energy effective theory that lives on the worldsheet of the string is the $\mathcal{N}=(2,2)$ $\mathbb{C P}^{N-1}$ sigma-model.

Since their discovery, BPS vortex-strings in $\mathcal{N}=2$ SQCD have been studied extensively. The study of the string moduli spaces and the string worldsheet theories, which preserve $\mathcal{N}=(2,2)$ supersymmetry on the worldsheet, has been generalized by adding flavors, by considering strings with general winding numbers and by considering other gauge groups. The methods one uses to study these strings range from brane constructions to explicit field theory derivations. For a partial list of references, see [3-14], and the reviews [15-18].

In the past decade, the quantum and non-perturbative understanding of theories with extended supersymmetry has progressed significantly due to the technique of supersymmetric localization. In particular, exact formulas are now available for squashed sphere partition functions of $4 d \mathcal{N}=2$ Lagrangian theories [19, 20], as well as of $2 d \mathcal{N}=(2,2)$ theories [21, 22], as long as the theories preserve appropriate $R$-symmetries. Fortunately, in the vortex-string set-up, both the parent $4 \mathrm{~d}$ theory and the $2 \mathrm{~d}$ worldsheet theory can be studied using localization techniques. This is due to the fact that the $4 \mathrm{~d}$ FI parameter, while breaking the $\mathrm{SU}(2)_{R}$ symmetry, preserves its Cartan, which is needed in order to preserve supersymmetry on the sphere. Similarly, the worldsheet theory inherits a vectorlike $R$-symmetry from the $4 \mathrm{~d}$ theory. This allows placing the theory on the sphere and computing its sphere partition function using supersymmetric localization. In this work, we will exploit the exact sphere partition function results to study the worldsheet theories of BPS vortex-strings, at the non-perturbative level, in new examples. For previous works that use localization results to study BPS vortex-strings, see [23-26]. In addition, many closely related works use supersymmetric localization to study surface defects coupled to $4 \mathrm{~d}$ theories. In particular, see the recent publications $[27,28]$ that share some similarities with the analysis that we present below.

We will generalize the well-studied vortex-strings, which are obtained when the U(1) flavor symmetry in which all the hypermultiplets have the same U(1) charge is gauged, by gauging, instead, a general U(1) flavor symmetry. In other words, we will study vortexstring configurations in a $4 \mathrm{~d} \mathcal{N}=2$ supersymmetric theory, with $\mathrm{SU}\left(N_{c}\right) \times \mathrm{U}(1)$ gauge group and $N_{f}$ hypermultiplets in the fundamental representation of $\mathrm{SU}\left(N_{c}\right)$, and with 
charges $c_{1}, \ldots, c_{N_{f}} \in \mathbb{Z}$ under the $\mathrm{U}(1)$ factor of the gauge group. ${ }^{1}$ The parameters of this theory are two complexified gauge couplings, one for the $\mathrm{SU}\left(N_{c}\right)$ factor of the gauge group and one for the $\mathrm{U}(1)$ factor of the gauge group, and an FI parameter $\xi$. In addition, we will introduce small, non-degenerate, hypermultiplet masses.

We will be interested in baryonic strings. These strings are labeled by the winding number $K$ and by a choice of a baryonic vacuum, in which scalars belonging to $N_{c}$ out of the $N_{f}$ hypermultiplets obtain vacuum expectation values (VEVs). The vacuum equations require that the sum of the U(1) charges of these $N_{c}$ hypermultiplets, which will be denoted by $C$, is non-vanishing. The string configuration carries magnetic flux $\Phi=2 \pi K / C$. Such fluxes are allowed due to a breaking of the $\mathrm{SU}\left(N_{c}\right) \times \mathrm{U}(1)$ gauge group to a residual $\mathbb{Z}_{C}$ subgroup in the baryonic vacuum.

For general $\mathrm{U}(1)$ charges, not all the strings will give rise to a $2 \mathrm{~d}$ effective theory on their worldsheet. The point is that when $N_{f}>N_{c}$ the bulk theory is not gapped and interactions between the bulk light fields and the worldsheet light fields are generally present. In [1] we studied these interactions, at the classical level, and argued that they are suppressed at low-energies if and only if

$$
\frac{\left(c_{i}-c_{j}\right) \Phi}{2 \pi}=\frac{\left(c_{i}-c_{j}\right) K}{C} \in \mathbb{Z} \text { for all } i, j . \quad \text { (Decoupling Criterion) }
$$

If $N_{f}=N_{c}$, the bulk theory is gapped. In this case, the low-energy dynamics is captured by a two-dimensional worldsheet theory, regardless of whether (1.1) is satisfied or not.

When $N_{f}>N_{c}$ and the condition (1.1) is not satisfied, the low-energy dynamics cannot be described in terms of a two-dimensional worldsheet theory, as one cannot separate the low-energy excitations to string excitations and bulk excitations. When (1.1) is satisfied, the classical analysis in [1] suggests that the string and the bulk decouple at low energies and it makes sense to discuss a $2 \mathrm{~d}$ worldsheet theory living on the string. The goal of this work will be to study the worldsheet theories that one obtains when the decoupling condition is satisfied (or when $N_{f}=N_{c}$ ). We do this by combining localization techniques on the four-ellipsoid with classical zero-mode analysis in flat space.

In order to apply localization techniques, we place the theory on the four-ellipsoid

$$
\frac{x_{0}^{2}}{r^{2}}+\frac{x_{1}^{2}+x_{2}^{2}}{l^{2}}+\frac{x_{3}^{2}+x_{4}^{2}}{\tilde{l}^{2}}=1 .
$$

The localization formula of [20] allows us to write a formal expression for the four-ellipsoid partition function, as a matrix integral over Coulomb branch coordinates. Unfortunately, this formal matrix integral is not well-defined - the Coulomb branch integral diverges due to the U(1) Landau pole. In order to define the partition function, we thus introduce an ultraviolet cut-off. That is, we cut-off the Coulomb branch integral below the scale set by the Landau pole.

For a wide range of parameters, we can close the cut-offed Coulomb branch integrals in the complex plane. In analogy to the Higgs branch localization in two dimensions [21, 22], we compute the integrals by summing over the residues of the encircled poles and obtain a

\footnotetext{
${ }^{1}$ Our analysis applies to any $N_{c} \geq 2$.
} 
representation of the four-ellipsoid partition function as a discrete sum, which we interpret as a sum over string contributions. This interpretation is motivated by the Higgs branch localization of $[23,24]$; the authors of these papers used a modified deformation term to localize the path integral for the four-ellipsoid partition function on saddle points that solve a BPS string-like equation, and argued that the representation that results from such a localization procedure can be obtained from the Coulomb branch representation of $[19,20]$ upon closing the contours in the complex plane. The interpretation of the residues of the poles of the integrand as encoding the string contributions is also motivated by the results of [29]. ${ }^{2}$

As will be explained in detail in sections 4 and 5, we obtain a representation of the four-ellipsoid partition function, which has the schematic form

$$
Z_{S_{b}^{4}}=\sum_{v \in \text { vacua }} e^{i \xi M_{v}} Z_{v}^{\text {bulk }} \sum_{K \in \mathbb{Z}} e^{-\xi \Phi_{K, v}} Z_{v, K}^{2 d}
$$

The sums in equation (1.3) are over the vacua, labeled by $v$, and the winding number $K$. The functions $Z_{v}^{\text {bulk }}$ and $Z_{v, K}^{2 d}$ are independent of the FI parameter $\xi$ and are uniquely defined as the coefficients in an expansion according to the dependence on $\xi$ and the requirement that $Z_{v, K=0}^{2 d}=1$. $\Phi_{K, v}$ is proportional to the magnetic flux of a string that is labeled by the vacuum $v$ and the winding number $K . M_{v}$ is related to the masses and charges of the fields that obtain an expectation value in the vacuum labeled by $v$. Let us emphasize that the expression (1.3) is a very schematic description of the representation that we find. In particular, we did not include in this schematic expression contributions of intersecting strings or contributions of mesonic vacua and strings. The detailed analysis and the exact expressions can be found in sections 4 and 5 .

$Z_{v}^{\text {bulk }}$ and $Z_{v, K}^{2 d}$ are functions of the rescaled hypermultiplet masses $\sqrt{i \tilde{l}} \mu_{i}, i=1, \ldots, N_{f}$, the gauge couplings, the theta parameters, and the squashing parameter $b=\sqrt{l / \tilde{l}}$. These functions are the output of our prescription. We now explain how we interpret this output.

$Z_{v}^{\text {bulk }}$ is the four-ellipsoid partition function of the light bulk fields in the vacuum $v$. We check this explicitly and compare to the spectrum in this vacuum. Exactly when the condition (1.1) is satisfied, the expression we find for $Z_{v, K}^{2 d}$ is of the form obtained in [21, 22] for two-sphere partition functions in the Higgs branch representation. Thus, when (1.1) is satisfied, we interpret $Z_{v, K}^{2 d}$ as the two-sphere partition function of the worldsheet theory of the $K$-string labeled by the vacuum $v$. The fact that only when (1.1) is satisfied the contributions factorize to a product of two decoupled factors - one describing the bulk fields and the other describing the worldsheet fields - is the supersymmetric localization derivation of the decoupling criterion (1.1), which was derived in [1] based on classical analysis of the interaction terms. One has to interpret the non-perturbative derivation of the decoupling criterion carefully, however, as there could still be $2 \mathrm{~d}-4 \mathrm{~d}$ interactions that are invisible to the sphere partition function.

The uniform U(1) charge example, which we work out in detail in section 4 , and in fact has already been studied using localization techniques in $[23,24,27,28]$, reproduces the

\footnotetext{
${ }^{2}$ In [29], residues of the superconformal index at poles in a $\mathrm{U}(1)$ flavor fugacity have been identified with the superconformal index of an infrared theory in the presence of surface defects. The surface defect is understood as the RG endpoint of a BPS vortex-string that exists when the U(1) flavor symmetry is gauged.
} 
worldsheet theory that appears in the literature, and is used as a test of our prescription. For general U(1) charges obeying (1.1), we obtain a formula for $Z_{v, K}^{2 d}$, which is expressed in terms of the parameters of the $4 \mathrm{~d}$ theory - see equation (5.12). This leaves us with the challenge of finding a $2 \mathrm{~d} \mathcal{N}=(2,2)$ theory with a two-sphere partition function that equals to $Z_{v, K}^{2 d}$ under some $4 \mathrm{~d}-2 \mathrm{~d}$ map of parameters.

In some cases, the weak coupling regime of the $4 \mathrm{~d}$ theory is mapped to the weak coupling regime of the $2 \mathrm{~d}$ theory, while in other cases, it is mapped to the strong coupling regime. The identification of the worldsheet theory in the latter case is significantly more challenging, as the expressions we obtain for $Z_{v, K}^{2 d}$ and the expressions for two-sphere partition functions that one finds in the localization literature, are all given as weak coupling expansions. Moreover, classical analysis of the string zero-modes, which provides useful insights in the former case, is less trustworthy in the latter case.

We propose a condition on the $\mathrm{U}(1)$ charges that guarantees that one can find a description of the worldsheet dynamics that is weakly coupled when the $4 \mathrm{~d}$ theory is weakly coupled: label the $N_{c}$ hypermultiplets getting vacuum expectation values by $a=1, \ldots, N_{c}$ and denote $C=\sum_{a=1}^{N_{c}} c_{a}$. The condition is that for each of the extra hypermultiplets, labeled by $i=N_{c}+1, \ldots, N_{f}$,

$$
\begin{array}{ll}
\Delta_{i a} \geq 0 & \text { for all } 1 \leq a \leq N_{c} \text { or } \\
\Delta_{i a} \leq-1 & \text { for all } 1 \leq a \leq N_{c},
\end{array}
$$

where

$$
\Delta_{i a}=\frac{c_{i}-c_{a}}{C}
$$

In terms of the classical zero-mode analysis, this is the condition that the $4 \mathrm{~d}$ F-term equations are satisfied trivially and do not impose any constraints on the target space of the worldsheet theory. See [1] and section 2.2. One can also derive this condition from our localization analysis, as the form of the general formula for the two-sphere partition function that we find changes dramatically when this condition is not satisfied. See section 5 .

In the class of strings defined by equation (1.4) we identify $Z_{v, K}^{2 d}$ with the two-sphere partition function of an $\mathcal{N}=(2,2)$ gauged linear sigma model (GLSM) with a $\mathrm{U}(|K|)$ gauge group. The matter content of this theory consists of one chiral multiplet in the adjoint representation, $N_{c}$ chiral multiplets in the fundamental representation and $N_{f}-$ $N_{c}$ chiral multiplets in the anti-fundamental representation. In addition, the spectrum contains neutral chiral multiplets, that are coupled to the fundamental, anti-fundamental and adjoint chiral multiplets via a superpotential, and extra decoupled chiral multiplets. The number of the neutral and the decoupled multiplets depends on the $U(1)$ charges and on the choice of the vacuum. The FI and theta parameters of the GLSM are given in terms of the Yang-Mills coupling and the theta angle of the $\mathrm{SU}\left(N_{c}\right)$ factor of the gauge group, as

$$
\xi_{2 d}=\frac{4 \pi}{g^{2}}, \quad \theta_{2 d}=\theta_{4 d}
$$

The worldsheet theory inherits two U(1) $R$-symmetries from the $4 \mathrm{~d}$ theory; one is related to the $4 \mathrm{~d} R$-symmetry and the other to rotations in the transverse plane to the 
string. From our localization analysis, we find the masses and the two types of $R$-charges of all the $2 \mathrm{~d}$ fields in terms of the $4 \mathrm{~d}$ parameters. In section 3 we test the proposal that the worldsheet theory is given by the low-energy limit of the GLSM we found, and the $4 \mathrm{~d}-2 \mathrm{~d}$ dictionary that we obtained, using classical analysis of the string zero-modes in flat space. We find perfect agreement between the spectrum that we obtain from localization and the spectrum that we obtain from the classical analysis.

It is generally hard to study the worldsheet theories when the $4 \mathrm{~d}-2 \mathrm{~d}$ dictionary maps the weak coupling regime to the strong coupling regime. However, in the $N_{f}=2 N_{c}=4$ case, one can relate strongly coupled worldsheet theories to weakly coupled worldsheet theories using four-dimensional $S$-duality. $\mathcal{N}=2$ supersymmetric $\mathrm{SU}(2)$ gauge theory with four fundamental massless hypermultiplets has an $\mathrm{SO}(8)$ flavor symmetry. The group $\mathrm{SO}(8)$ has an $\mathbf{S}_{\mathbf{3}}$ outer automorphism group, which acts as the $S$-duality group of the massive theory [30]. The six elements of $\mathbf{S}_{\mathbf{3}}$ can be generated using the transformations

$$
\begin{aligned}
& T: \quad q \rightarrow \frac{q}{q-1}, \quad S: \quad q \rightarrow 1-q, \\
& \mu_{1} \rightarrow \mu_{1}, \quad \mu_{1} \rightarrow \frac{1}{2}\left(\mu_{1}+\mu_{2}+\mu_{3}+\mu_{4}\right), \\
& \mu_{2} \rightarrow \mu_{2}, \quad \mu_{2} \rightarrow \frac{1}{2}\left(\mu_{1}+\mu_{2}-\mu_{3}-\mu_{4}\right), \\
& \mu_{3} \rightarrow \mu_{3}, \quad \mu_{3} \rightarrow \frac{1}{2}\left(\mu_{1}-\mu_{2}+\mu_{3}-\mu_{4}\right), \\
& \mu_{4} \rightarrow-\mu_{4} \quad \mu_{4} \rightarrow \frac{1}{2}\left(\mu_{1}-\mu_{2}-\mu_{3}+\mu_{4}\right),
\end{aligned}
$$

where $q=e^{2 \pi i \tau_{s u(2)}}$ and $\mu_{1}, \ldots, \mu_{4}$ are the hypermultiplet masses.

In order to allow string configurations, we need to gauge a $U(1)$ flavor symmetry. The $\mathrm{U}(1)$ charges of the hypermultiplets, $c_{1}, \ldots, c_{4}$, map under the $\mathbf{S}_{\mathbf{3}}$ transformations in the same way the masses map in equation (1.7). This provides us with a prediction for the worldsheet theories on strings that are related by these transformations to strings that satisfy condition (1.4); the worldsheet theory has to be the same, but the $4 \mathrm{~d}-2 \mathrm{~d}$ map of the parameters is modified according to the map (1.7). In particular, applying the $S$ transformation to strings that satisfy (1.4), one obtains $4 \mathrm{~d}-2 \mathrm{~d}$ dictionaries that map the weak coupling regime to the strong coupling regime. Indeed, in section 6.2 we show, for winding number $K=1$, that the expression we find for $Z_{v, K}^{2 d}$ gives the expected partition functions for charge assignments that are related by triality to the equal charge case. This check is enabled due to hypergeometric function identities.

Let us end the introduction with a comment about the limitations of our analysis. The two-sphere partition function does not capture all the on the theory. As a result, one cannot uniquely identify the worldsheet theory based on this observable. In the equal charge case, for example, the output of our prescription agrees with the Hanany-Tong model $[3,6]$, which is believed to reproduce correctly various observables that are protected by supersymmetry. However, it is known, for example, that the target space metric of the Hanany-Tong model is not the correct target space metric for winding number $K>1$ [31]. 
While the exact worldsheet theory cannot be determined uniquely from its sphere partition function, a large amount of non-trivial information can be extracted from this observable. To enrich the sphere partition function, we include generic values for all the possible parameters. As a result, the output of our localization computation depends on many parameters - the hypermultiplet masses and U(1) charges, the ellipsoid squashing parameter, the $\mathrm{SU}\left(N_{c}\right)$ gauge coupling and the $\mathrm{SU}\left(N_{c}\right)$ theta angle. This makes the agreement between the expressions we compare highly non-trivial. Moreover, whenever possible, we compare the spectrum of the worldsheet theory we obtain to expectations based on classical zero-mode analysis.

The outline of the paper is as follows. In section 2 we review the classical vacuum and string equations and some of the properties of the string moduli space. Most of the content of this section already appeared in [1]. In section 3 we list our proposals for the worldsheet theories on strings that satisfy conditions (1.1) and (1.4) and explain the spectrum that we find from the classical point of view. In section 4 we derive the Higgs branch representation of the four-ellipsoid partition function in the $c_{i}=1$ case and explain how to read from this expression the two-sphere partition functions of the worldsheet theories. In section 5 we generalize the derivation of section 4 to theories with arbitrary $\mathrm{U}(1)$ charges. We give a closed formula for the two-sphere partition function of the worldsheet theory that is applicable in all the cases in which the string decouples from the bulk. In the cases that satisfy condition (1.4), we identify the two-sphere partition function with the two-sphere partition function of the GLSMs presented in section 3. Section 6 is devoted to the triality - the S-duality in the $N_{f}=2 N_{c}=4$ case. We explain the predictions for the worldsheet theories that one can derive from the triality and use our localization prescription to verify these predictions for strings that are related by triality to the minimal strings in the equal charge case. Technical details are collected in four appendices.

\section{The classical string equations and the string moduli space}

In this section, we will review the classical vacuum and string equations and some of the properties of the string moduli space. This analysis is done in flat spacetime. Most of the content of this section already appeared in [1].

\subsection{The classical vacuum and string equations}

Our starting point is a $4 \mathrm{~d} \mathcal{N}=2$ supersymmetric gauge theory with $\mathrm{SU}\left(N_{c}\right) \times \mathrm{U}(1)$ gauge group and $N_{f}$ hypermultiplets in the fundamental representation of $\mathrm{SU}\left(N_{c}\right)$. We will assume that $N_{c} \leq N_{f} \leq 2 N_{c}$. The charges of the hypermultiplets under the U(1) factor of the gauge group will be denoted by $c_{1}, c_{2}, \ldots, c_{N_{f}} \in \mathbb{Z}$. We will denote the complexified gauge couplings of the $\mathrm{SU}\left(N_{c}\right)$ and $\mathrm{U}(1)$ factors of the gauge group by

$$
\tau_{u(1)}=\frac{\theta_{u(1)}}{2 \pi}+\frac{4 \pi i}{e^{2}}, \quad \tau_{s u(N)}=\frac{\theta}{2 \pi}+\frac{4 \pi i}{g^{2}}
$$


To force the vacuum into the Higgs branch, we will introduce a Fayet-Ilioupulus parameter $\xi>0$. In addition, we will introduce non-degenerate hypermultiplet masses $\mu_{i} \ll e \sqrt{\xi}, g \sqrt{\xi}^{3}{ }^{3}$

The bosonic part of the action is given by

$$
\begin{aligned}
S= & \int d^{4} x\left[\frac{1}{4 g^{2}}\left(F_{\mu \nu}^{\alpha}\right)^{2}+\frac{1}{4 e^{2}}\left(F_{\mu \nu}^{\prime}\right)^{2}+\frac{1}{g^{2}}\left|\mathcal{D}_{\mu} a^{\alpha}\right|^{2}+\frac{1}{e^{2}}\left|\partial_{\mu} a^{\prime}\right|^{2}+\sum_{i}\left|\mathcal{D}_{\mu} q_{i}\right|^{2}+\sum_{i}\left|\mathcal{D}_{\mu} \tilde{q}^{i}\right|^{2}-V+\mathcal{L}_{\theta}\right], \\
V= & \frac{g^{2}}{2}\left(\frac{1}{g^{2}} f^{\alpha \beta \gamma} a_{\beta}^{\dagger} a_{\gamma}+\sum_{i} q^{i \dagger} \lambda^{\alpha} q_{i}-\sum_{i} \tilde{q}^{i} \lambda^{\alpha} \tilde{q}_{i}^{\dagger}\right)^{2}+\frac{e^{2}}{8}\left(\sum_{i} c_{i} q^{i \dagger} q_{i}-\sum_{i} c_{i} \tilde{q}_{i} \tilde{q}^{i \dagger}-N_{c} \xi\right)^{2} \\
& +2 g^{2}\left|\sum_{i} \tilde{q}^{i} \lambda^{\alpha} q_{i}\right|^{2}+\frac{e^{2}}{2}\left|\sum_{i} c_{i} \tilde{q}^{i} q_{i}\right|^{2}+\sum_{i}\left|\left(c_{i} a^{\prime}+\lambda^{\alpha} a^{\alpha}+\mu_{i}\right) q_{i}\right|^{2}+\sum_{i}\left|\left(c_{i} a^{\prime}+\lambda^{\alpha} a^{\alpha}+\mu_{i}\right) \tilde{q}_{i}^{\dagger}\right|^{2}, \\
\mathcal{L}_{\theta}= & \frac{\theta}{32 \pi^{2}} F_{\mu \nu}^{\alpha} \tilde{F}^{\alpha \mu \nu}+\frac{\theta_{u(1)}}{32 \pi^{2}} F_{\mu \nu}^{\prime} \tilde{F}^{\prime \mu \nu},
\end{aligned}
$$

where

$$
\begin{aligned}
\mathcal{D}_{\mu} q_{i} & =\left(\partial_{\mu}-i c_{i} A_{\mu}^{\prime}-i \lambda^{\alpha} A_{\mu}^{\alpha}\right) q_{i}, \quad \mathcal{D}_{\mu} \tilde{q}_{i}^{\dagger}=\left(\partial_{\mu}-i c_{i} A_{\mu}^{\prime}-i \lambda^{\alpha} A_{\mu}^{\alpha}\right) \tilde{q}_{i}^{\dagger}, \\
\mathcal{D}_{\mu} a^{\alpha} & =\left(\partial_{\mu} \delta^{\alpha \gamma}-i f^{\alpha \beta \gamma} A_{\beta, \mu}\right) a_{\gamma} .
\end{aligned}
$$

In the equation above, the scalar and the gauge field of the $\mathrm{U}(1)$ vector multiplet are denoted by $a^{\prime}$ and $A_{\mu}^{\prime}$ respectively. Similarly, the scalars and gauge fields of the $\operatorname{SU}\left(N_{c}\right)$ vector multiplet are denoted by $a^{\alpha}$ and $A_{\mu}^{\alpha}, \alpha=1, \ldots, N_{c}^{2}-1$. The generators and structure constants of $\mathrm{SU}\left(N_{c}\right)$ are denoted by $\lambda^{\alpha}$ and $f^{\alpha \beta \gamma} . q_{i}^{a}$ and $\tilde{q}_{a}^{i}$ are the hypermultiplets scalars, where $i=1, \ldots, N_{f}$ is the flavor index and the $a=1, \ldots, N_{c}$ is the color index. The color index was suppressed in (2.2). In some of the equations below, the hypermultiplet scalars will be organized as matrices,

$$
\mathbf{q}=\left(\begin{array}{cccc}
q_{1}^{1} & q_{2}^{1} & \cdots & q_{N_{f}}^{1} \\
q_{1}^{2} & q_{2}^{2} & \cdots & q_{N_{f}}^{2} \\
\vdots & \vdots & \ddots & \vdots \\
q_{1}^{N_{c}} & q_{2}^{N_{c}} & \cdots & q_{N_{f}}^{N_{c}}
\end{array}\right), \tilde{\mathbf{q}}=\left(\begin{array}{cccc}
\tilde{q}_{1}^{1} & \tilde{q}_{2}^{1} & \cdots & \tilde{q}_{N_{c}}^{1} \\
\tilde{q}_{1}^{2} & \tilde{q}_{2}^{2} & \cdots & \tilde{q}_{N_{c}}^{2} \\
\vdots & \vdots & \ddots & \vdots \\
\tilde{q}_{1}^{N_{f}} & \tilde{q}_{2}^{N_{f}} & \cdots & \tilde{q}_{N_{c}}^{N_{f}}
\end{array}\right)
$$

In the absence of the FI parameter, the theory enjoys a non-anomalous $\mathrm{SU}(2)_{R} R$ symmetry, which is broken by the FI parameter to its Cartan. The global symmetry of the theory is $\mathrm{U}(1)^{N_{f}-1}$. If some of the $\mathrm{U}(1)$ charges $c_{i}$ are equal, the theory enjoys an approximate non-abelian global symmetry which is broken by the small hypermultiplet masses.

The vacua of the theory are labeled by $\mathrm{SU}\left(N_{c}\right)$ invariant operators that are charged under the $\mathrm{U}(1)$ factor of the gauge group. For generic $c_{i}$, both charged mesons and charged baryons exist. This leads to two types of vacua - mesonic and baryonic.

The mesonic vacua are given, up to gauge transformations, by

$$
q_{i}^{1}=\tilde{q}_{1}^{j}=\sqrt{\frac{N_{c} \xi}{c_{i}-c_{j}}}, \quad c_{i}>c_{j} .
$$

\footnotetext{
${ }^{3}$ Masses of charged hypermultiplets can be shifted arbitrarily by redefinitions of the U(1) gauge multiplet scalar. What we need to assume is actually $\left|\mu_{i}-\mu_{a}+\Delta_{a i} \sum_{b=1}^{N_{c}} \mu_{b}\right| \ll e \sqrt{\xi}, g \sqrt{\xi}$.
} 
In addition, the adjoint scalars need to satisfy the constraints

$$
c_{i} a^{\prime}+\lambda_{11}^{\alpha} a^{\alpha}+\mu_{i}=c_{j} a^{\prime}+\lambda_{11}^{\alpha} a^{\alpha}+\mu_{j}=0 .
$$

Thus, the mesonic vacua have $N_{c}-2$ flat Coulomb branch directions. The vacuum (2.5) breaks the gauge symmetry to $\mathbb{Z}_{\left|c_{i}-c_{j}\right|} \times \mathrm{SU}\left(N_{c}-1\right)$. We will not discuss mesonic vacua and strings in this work, with one exception - in the $N_{f}=2 N_{c}=4$ example that we study in section 6.2 .3 , we will study also mesonic strings that are related by $S$-duality to baryonic strings in a different theory.

We will focus on the baryonic vacua and the corresponding strings. The baryonic vacua are labeled by choices of $N_{c}$ out of the $N_{f}$ hypermultiplets such that the sum of their charges is not zero. Without loss of generality, we will choose the first $N_{c}$ hypermultiplets and assume that $C \equiv \sum_{i=1}^{N_{c}} c_{i}>0$ (flipping the sign of $C$ amounts to replacing $q \leftrightarrow \tilde{q}$ ).

Up to gauge transformations, the vacuum corresponding to this choice of hypermultiplets is given by

$$
\begin{array}{lc}
\mathbf{q}=\left(v \mathbb{I}_{N_{c} \times N_{c}} 0_{\left(N_{f}-N_{c}\right) \times N_{c}}\right), & v^{2}=\frac{N_{c} \xi}{C}, \\
c_{b} \delta_{a b} a^{\prime}+\lambda_{a b}^{\alpha} a^{\alpha}+\mu_{b} \delta_{a b}=0, & a, b=1, \ldots, N_{c},
\end{array}
$$

where all the other fields vanish. The gauge symmetry is broken in the vacuum to a $\mathbb{Z}_{C}$ subgroup that acts on the scalars as

$$
q_{i}^{a} \rightarrow e^{2 \pi i n \Delta_{i a}} q_{i}^{a}, \quad n=1,2, \ldots, C .
$$

The masses of the $q_{i}^{a}$ excitations in the vacuum (2.7) are given by

$$
\mu_{i a}^{2}=\left(\mu_{i}-\mu_{a}+\Delta_{a i} \sum_{b=1}^{N_{c}} \mu_{b}\right)^{2}
$$

where

$$
\Delta_{a i}=\frac{c_{a}-c_{i}}{C}
$$

The vacuum preserves a combination of the $\mathrm{U}(1) \subset \mathrm{SU}(2)_{R}$ that is preserved in the presence of the FI term and an $\mathrm{SU}\left(N_{c}\right) \times \mathrm{U}(1)$ transformation. The charges of the hypermultiplet scalars under this combination are given by

$$
R^{(R)}\left[q_{i}^{a}\right]=N_{c} \Delta_{a i}, \quad R^{(R)}\left[\tilde{q}_{a}^{i}\right]=2+N_{c} \Delta_{i a} .
$$

Topologically stable strings are thus characterized by the homotopy group $\pi_{1}(\mathrm{U}(1) \times$ $\left.\mathrm{SU}\left(N_{c}\right) / \mathbb{Z}_{C}\right)$, which allows fluxes quantized as

$$
\Phi=\lim _{r \rightarrow \infty} \int d \phi A_{\phi}^{\prime}=\frac{2 \pi K}{C}, \quad K \in \mathbb{Z} .
$$

The spectrum in the vacuum consists of $N_{c}\left(N_{f}-N_{c}\right)$ light hypermultiplets, with masses given by equation (2.9) for $i=N_{c}+1, \ldots, N_{f}, a=1, \ldots, N_{c}$. In addition, the $N_{c}^{2}$ gauge 
multiplets combine with the $N_{c}^{2}$ hypermultiplets labeled by $i \leq N_{c}$ via the Higgs mechanism to create $N_{c}^{2}$ long massive vector multiplets, with masses $m_{W} \sim e \sqrt{\xi}, g \sqrt{\xi}$.

Let us now derive the string equations. We will take the string to lie along the $x^{3}$ direction. Assuming that the configuration does not depend on the worldsheet coordinates $x^{0}$ and $x^{3}$, that $A_{0}, A_{3}$ and the off-diagonal elements of $a$ vanish, and that $a$ and $a^{\prime}$ are constant, the tension can be written as

$$
\begin{aligned}
T= & \int d x^{1} d x^{2}\left[\frac{1}{2 e^{2}}\left(B_{3}^{\prime} \pm \frac{e^{2}}{2}\left(\sum_{i} c_{i}\left|q_{i}\right|^{2}-\sum_{i} c_{i}\left|\tilde{q}_{i}\right|^{2}-N_{c} \xi\right)\right)^{2}+2 g^{2}\left|\sum_{i} \tilde{q}^{i} \lambda^{\alpha} q_{i}\right|^{2}+\frac{e^{2}}{2}\left|\sum_{i} c_{i} \tilde{q}^{i} q_{i}\right|^{2}\right. \\
& +\frac{1}{2 g^{2}}\left(B_{3}^{\alpha} \pm g^{2} \sum_{i} q^{i \dagger} \lambda^{\alpha} q_{i} \mp g^{2} \sum_{i} \tilde{q}^{i} \lambda^{\alpha} \tilde{q}_{i}^{\dagger}\right)^{2}+\sum_{i}\left|\mathcal{D}_{1} q_{i} \pm i \mathcal{D}_{2} q_{i}\right|^{2}+\sum_{i}\left|\mathcal{D}_{1} \tilde{q}_{i} \pm i \mathcal{D}_{2} \tilde{q}^{i}\right|^{2} \\
& \left.+\sum_{i}\left|\left(c_{i} a^{\prime}+\lambda^{\alpha} a^{\alpha}+\mu_{i}\right) q_{i}\right|^{2}+\sum_{i}\left|\left(c_{i} a^{\prime}+\lambda^{\alpha} a^{\alpha}+\mu_{i}\right) \tilde{q}_{i}^{\dagger}\right|^{2} \pm N_{c} \xi B_{3}^{\prime}\right] \\
\geq & \pm \int d x^{1} d x^{2} N_{c} \xi B_{3}^{\prime},
\end{aligned}
$$

where $B_{3}^{\prime}=F_{12}^{\prime}, B_{3}^{\alpha}=F_{12}^{\alpha}$. The last term in this expression is a topological term that is proportional to the magnetic flux. The Bogomol'nyi equations are obtained by requiring the vanishing of the other terms:

$$
\begin{aligned}
& B_{3}^{\prime} \pm \frac{e^{2}}{2}\left(\sum_{i} c_{i}\left|q_{i}\right|^{2}-\sum_{i} c_{i}\left|\tilde{q}_{i}\right|^{2}-N_{c} \xi\right)=B_{3}^{\alpha} \pm g^{2} \sum_{i} q^{i \dagger} \lambda^{\alpha} q_{i} \mp g^{2} \sum_{i} \tilde{q}^{i} \lambda^{\alpha} \tilde{q}_{i}^{\dagger}=0, \\
& \sum_{i} \tilde{q}^{i} \lambda^{\alpha} q_{i}=\sum_{i} c_{i} \tilde{q}^{i} q_{i}=\mathcal{D}_{1} q_{i} \pm i \mathcal{D}_{2} q_{i}=\mathcal{D}_{1} \tilde{q}_{i} \pm i \mathcal{D}_{2} \tilde{q}^{i}=0, \\
& \left(c_{i} a^{\prime}+\lambda^{\alpha} a^{\alpha}+\mu_{i}\right) q_{i}=\left(c_{i} a^{\prime}+\lambda^{\alpha} a^{\alpha}+\mu_{i}\right) \tilde{q}_{i}^{\dagger}=0 .
\end{aligned}
$$

Solutions to these equations minimize the tension in a given topological sector and give rise to $1 / 2$-BPS strings.

We will focus on configurations with positive magnetic flux in the $x_{3}$ direction, by picking the upper sign in (2.13), (2.14). One can look for string solutions that satisfy the ansatz

$$
\begin{aligned}
q_{i}^{a}=q_{a}(r, \phi) \delta_{i}^{a}, & \lim _{r \rightarrow \infty} q_{a}(r, \phi) & =v e^{i k_{a} \phi}, \\
c_{b} \delta_{a b} a^{\prime}+\lambda_{a b}^{\alpha} a^{\alpha}+\mu_{b} \delta_{a b}, & a, b & =1, \ldots, N_{c} .
\end{aligned}
$$

where $r, \phi$ are the polar coordinates on the $x^{1}-x^{2}$ plane and $k_{a}$ are non-negative integers. For such a solution to exist, the diagonal elements of the gauge field need to be turned on to satisfy (2.14). All other fields vanish. The magnetic flux carried by a solution of the Bogomol'nyi equations with an asymptotic behavior of the form (2.15) is given by

$$
\Phi=\lim _{r \rightarrow \infty} \int A_{\phi}^{\prime} d \phi=\frac{2 \pi K}{C}
$$

where $K \equiv \sum_{a=1}^{N_{c}} k_{a}$ is the topological charge of the string. 


\section{$2.2 \quad$ The string moduli}

To discuss the string solution, one can start with an ansatz of the form (2.15) and plug it into the Bogomol'nyi equations. The solution for $q_{a}(r, \phi)$ is guaranteed to have $k_{a}$ zeros [32]. The string solution has translational zero-modes, related to the locations of the zeros. In the presence of generic hypermultiplet masses these are expected to be the only exact zero-modes.

However, we will be interested in fluctuations above the string solution with energies in the range $\mu_{i a} \lesssim E \ll e \sqrt{\xi}, g \sqrt{\xi}$. Therefore, we need to consider all the deformations of the string solution that become true zero-modes in the massless limit - these will be treated as approximate zero-modes in the $\mu_{i a} \ll e \sqrt{\xi}, g \sqrt{\xi}$ limit. In other words, we consider only the first two lines of (2.14) as the string equations. Fixing the vector multiplet scalars on their vacuum expectation values, the terms in the third line of (2.14) will give rise to small masses for the worldsheet fields. For the tension cost of such deformations to be finite (and small), and to avoid problems related to non-normalizability when the quasi-moduli are promoted to fields on the worldsheet, one has to modify these modes at distances above $\sim \frac{1}{\mu_{i a}}$ (as in [7], for example). This does not alter the fact that these modes approach zero-modes of the string in the massless limit. For a more detailed discussion, see [1].

Global symmetries of the massless theory can be used to generate new approximate solutions from a given string solution. However, not all the quasi-moduli are of this form. A crucial role is played by moduli that will be referred to as size-moduli. These are deformations of the string solution that exist only when $N_{f}>N_{c}$ and affect the size of the string in the transverse plane.

Let us consider a string solution that has the diagonal form (2.15) for $i \leq N_{c}$. In contrast to equation (2.15), however, we now allow the $i>N_{c}$ entries of $\mathbf{q}$ and $\tilde{\mathbf{q}}$ to be nonvanishing. In [1] we showed that the Bogomol'nyi equations and the boundary conditions imply the following relation between the $i>N_{c}$ and $i \leq N_{c}$ entries:

$$
\begin{aligned}
& q_{i>N_{c}}^{a}=\left(\prod_{b \neq a} \frac{q_{b}(r, \phi)}{\prod_{l_{b}=1}^{k_{b}}\left(z-z_{l_{b}}\right)}\right)^{\Delta_{i a}}\left(\frac{q_{a}(r, \phi)}{\prod_{l_{a}=1}^{k_{a}}\left(z-z_{l_{a}}\right)}\right)^{1+\Delta_{i a}} \times \sum_{n=0}^{\left\lceil k_{a}+\Delta_{i a} K\right\rceil-1} \rho_{i a}^{(n)} z^{n}, \\
& \tilde{q}_{a}^{i>N_{c}}=\left(\prod_{b \neq a} \frac{q_{b}(r, \phi)}{\prod_{l_{b}=1}^{k_{b}}\left(z-z_{l_{b}}\right)}\right)^{-\Delta_{i a}}\left(\frac{q_{a}(r, \phi)}{\left.\prod_{l_{a}=1}^{k_{a}\left(z-z_{l_{a}}\right)}\right)^{-\Delta_{i a}-1} \times \sum_{n=0}^{\left\lceil-k_{a}-\Delta_{i a} K\right\rceil-1} \tilde{\rho}_{i a}^{(n)} z^{n},}\right.
\end{aligned}
$$

where $z_{l_{a}}, l_{a}=1, \ldots, k_{a}$, are the zeros of $q_{a}(r, \phi) . \rho_{i a}^{(n)}$ and $\tilde{\rho}_{i a}^{(n)}$ are complex parameters that will be referred to as the size-moduli of the string. When these moduli exist, the string solution approaches the large $r$ limit of (2.15) with a power-law behavior, with typical size that is determined by the size-moduli (see appendix A of [1]).

From equation (2.17) one can see that long-range moduli for which $q^{i>N_{c}}$ or $\tilde{q}_{i>N_{c}}$ decay slower than $1 / r$ exist if and only if $\Delta_{i a} K \notin \mathbb{Z}$ for some $i>N_{c}$ and $a \leq N_{c}$. In [1] we argued that these are exactly the cases in which the string and the bulk moduli do not 
decouple at low energies. ${ }^{4}$ For a supersymmetric localization derivation of this condition, see section 5. In this work, we wish to identify the worldsheet theories on strings that decouple from the bulk. Thus, we will assume that $\Delta_{i a} K \in \mathbb{Z}$.

When for some flavor both $q$ and $\tilde{q}$ can be excited, the F-term equations

$$
\sum_{i} \tilde{q}^{i} \lambda^{\alpha} q_{i}=\sum_{i} c_{i} \tilde{q}^{i} q_{i}=0
$$

act as extra constraints on the moduli space. For strings that satisfy the condition (1.4), one cannot excite $q$ and $\tilde{q}$ together for the same flavor, as can be seen in equation (2.17). For these strings, we find a description of the worldsheet theory that is weakly coupled when the four-dimensional theory is weakly coupled. We discuss these cases in the next section.

If $n$ of the charges $c_{1}, \ldots, c_{N_{c}}$ are equal, the theory has a global $\mathrm{SU}(n)$ symmetry in the massless limit, acting on the equally charged hypermultiplets. This symmetry can be used to generate new string solutions. The moduli associated with this symmetry sit in the off-diagonal elements of $q_{a}^{b}$ with $a, b \leq N_{c}$ and $a \neq b$. However, off-diagonal components of $q_{a}^{b}$ can give rise to zero-modes even when no global symmetry is involved. Evidence for the existence of these modes for strings that satisfy the condition (1.4) come from our localization results, and are supported by expectations from $S$-duality (see appendix D). When the condition (1.4) is satisfied, we expect each $a, b$ pair to give rise to $k_{a}+k_{b}$ complex moduli with masses $\mu_{a b}$ given by equation (2.9). ${ }^{5}$

Due to the $1 / 2$-BPS nature of the string solutions, the worldsheet theory is $\mathcal{N}=(2,2)$ supersymmetric. In addition, the worldsheet theory inherits a vectorlike R-symmetry and a flavor symmetry from the four-dimensional theory.

As usual, the R-symmetry can mix with a U(1) flavor symmetry. For the sake of the comparison with the localization analysis, it will be convenient for us to write the R-charges as $R_{2 d}=\alpha R^{(J)}+(1-\alpha) R^{(R)}, \alpha \in \mathbb{R}$, where in terms of the symmetries of the $4 \mathrm{~d}$ theory $R^{(R)}$ is related to the $\mathrm{U}(1) R$-symmetry generator preserved by the string background and $R^{(J)}$ is related to rotations in the $x^{1}-x^{2}$ plane. When normalized as a worldsheet $R$-symmetry generator, the charges of the hypermultiplet scalars under $R^{(R)}$ are given by

$$
R^{(R)}\left[q_{i}^{a}\right]=N_{c} \Delta_{a i}, \quad R^{(R)}\left[\tilde{q}_{a}^{i}\right]=2+N_{c} \Delta_{i a} .
$$

$R^{(J)}$ is related to rotations in the $x^{1}-x^{2}$ plane in the following way. A generic string configuration breaks rotations symmetry in the $x^{1}-x^{2}$ plane. However, a string solution of the form

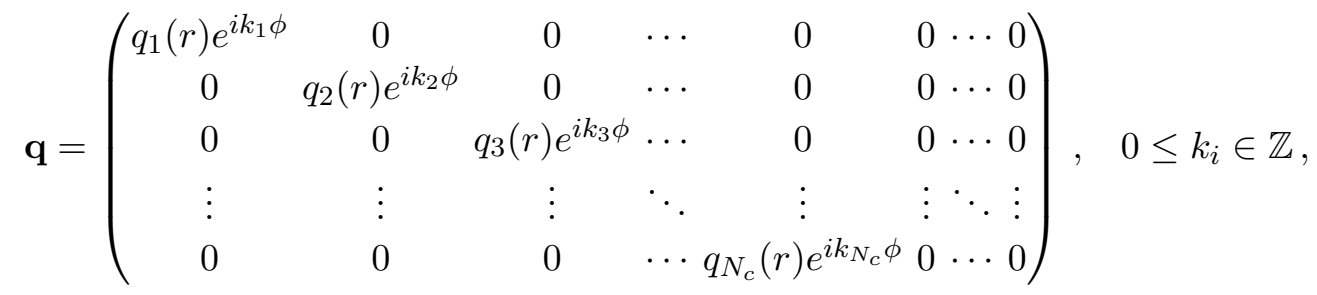

\footnotetext{
${ }^{4}$ The decoupling criterion deduced from equation (2.17) is $\Delta_{i a} K \in \mathbb{Z}$ for all $a \leq N_{c}<i$. By taking differences for different $i$ 's or different $a$ 's we obtain the condition (1.1).

${ }^{5}$ One can show that an excitation of $q_{a}^{b}$ should be accompanied by an excitation of $q_{b}^{a}$. The first line in (2.14) implies that an excitation of $q_{a}^{b}$ must be accompanied with excitation of $A_{a b}$ and $A_{b a}$. The Bogomol'nyi equation $\left(\mathcal{D}_{1}+i \mathcal{D}_{2}\right) q=0$ therefore requires that $q_{b}^{a} \neq 0$.
} 
preserves a combination of rotations and gauge transformations. Normalized as $R$-symmetry on the worldsheet, this transformation acts on the scalars $q_{i}^{a}$ and the angle $\phi$ as $^{6}$

$$
q_{i}^{a} \rightarrow e^{2 i k_{a} \phi_{0}+2 i K \Delta_{i a} \phi_{0}} q_{i}^{a}, \phi \rightarrow \phi-2 \phi_{0}
$$

The $R^{(J)}$-charges of the size-modes are thus given by

$$
R^{(J)}\left[\rho_{i a}^{(n)}\right]=2 K \Delta_{i a}+2 k_{a}-2 n, \quad R^{(J)}\left[\tilde{\rho}_{i a}^{(n)}\right]=-2 K \Delta_{i a}-2 k_{a}-2 n .
$$

\section{Examples of worldsheet theories}

In this section we discuss the worldsheet theories on strings that satisfy, in addition to the decoupling condition (1.1), the following condition: for every $N_{c}<i \leq N_{f}$,

$$
\begin{array}{ll}
\Delta_{i a} \geq 0 & \text { for all } 1 \leq a \leq N_{c} \text { or } \\
\Delta_{i a} \leq-1 & \text { for all } 1 \leq a \leq N_{c} .
\end{array}
$$

These are the cases in which there is no flavor for which both $q_{i}$ and $\tilde{q}^{i}$ give rise to zeromodes and therefore the F-terms $\sum_{i} \tilde{q}^{i} \lambda^{\alpha} q_{i}, \sum_{i} c_{i} \tilde{q}^{i} q_{i}$ vanish identically, without imposing any additional constraints on the target space.

For strings that satisfy the condition (3.1), we identify in section 5 a two-dimensional GLSM whose two-sphere partition function matches exactly with the output of our localization analysis. The corresponding low-energy non-linear sigma model (NLSM) provides a proposal for the worldsheet theory. The map between the $4 \mathrm{~d}$ and $2 \mathrm{~d}$ couplings that we obtain in these cases is such that the worldsheet GLSM is weakly coupled when the four-dimensional theory is weakly coupled.

In this section we will describe our proposal for the worldsheet theories and will test it against expectations that are based on classical zero-mode analysis. We will start with the special case where $c_{i}=1$ for all $i=1, \ldots, N_{c}$. Then, in section 3.2 , we will generalize to the case where $c_{i} \geq c_{a}$ for all $1 \leq a \leq N_{c}$ and $N_{c}+1 \leq i \leq N_{f}$. Finally, in section 3.3, we will discuss the most general case satisfying the condition (3.1).

\subsection{The equal charge case}

The case in which $c_{i}=1$ for every $i=1, \ldots, N_{f}$ has been studied extensively in the literature. See, for example, [3-9], and the reviews [15-18]. The worldsheet theory has been studied in various methods, such as brane construction [3, 6], matching of the BPS spectra [2, 12], moduli matrix approach [8, 18], as well as explicit derivations in field theory $[4-7,9]$ and localization techniques (see, for example, $[23,24]$ and section 4.2 of this work.)

\footnotetext{
${ }^{6}$ Under a general Cartan gauge transformation, $q_{i}^{a} \rightarrow e^{i c_{i} \omega^{\prime}+i \omega_{a}} q_{i}^{a}$ with $\sum_{a=1}^{N_{c}} \omega_{a}=0$. Equation (2.20) is invariant under $\phi \rightarrow \phi+\delta \phi$ if we accompany this transformation with the gauge transformation parametrized by $\omega^{\prime}=-\frac{K \delta \phi}{C}, \omega_{a}=-k_{a} \delta \phi+\frac{K c_{a} \delta \phi}{C}$.
} 


\begin{tabular}{|c|c|c|c|c|}
\hline Field & $\mathrm{U}(K)$ Representation & Twisted Mass & $R^{(J)}$ & $R^{(R)}$ \\
$X$ & adjoint & 0 & 2 & 0 \\
$\psi_{a}$ & fundamental & $\mu_{a}$ & 2 & 0 \\
$\tilde{\psi}_{i}$ & anti-fundamental & $-\mu_{i}$ & 0 & 0 \\
\hline
\end{tabular}

Table 1. The spectrum of the worldsheet GLSM in the equal charge case.

For a string solution in which the hypermultiplet scalars labeled by $a=1, \ldots, N_{c}$ obtain a vacuum expectation value, Hanany and Tong proposed that the worldsheet theory can be described as the low-energy limit of an $\mathcal{N}=(2,2) \mathrm{U}(K)$ gauge theory with

- 1 massless chiral multiplet, $X$, in the adjoint representation of $\mathrm{U}(K)$,

- $N_{c}$ chiral multiplets, $\psi_{a}, a=1, \ldots, N_{c}$, in the fundamental representation of $\mathrm{U}(K)$,

- $N_{f}-N_{c}$ chiral multiplets, $\tilde{\psi}_{i}, i=N_{c}+1, \ldots, N_{f}$, in the anti-fundamental representation of $\mathrm{U}(K)$.

The FI parameter of this theory is given in terms of Yang-Mills coupling of $\mathrm{SU}\left(N_{c}\right)$ factor of the gauge group via

$$
\xi_{2 d}=\frac{4 \pi}{g^{2}} .
$$

The localization analysis of $[23,24]$ relates the $\theta$-parameters in the following way:

$$
\theta_{2 d}=\theta_{4 d}
$$

The twisted masses and $R$-charges are summarized in table $1 .{ }^{7}$

The NLSM fields that arise from the GLSM fundamental chiral multiplets are related to vacuum symmetries that are broken by the string solutions. The anti-fundamental chiral multiplets consist of the size-moduli and their superpartners, while the adjoint chiral multiplet consists of the translational moduli and their superpartners. Since the latter are exact zero-modes, the adjoint chiral multiplet is massless.

\subsection{Strings with no $\tilde{q}$ excitations}

\subsubsection{Proposal for the worldsheet theory}

We now relax the equal charge requirement, and instead require that

$$
c_{i} \geq c_{a} \text { for all } a \leq N_{c}<i .
$$

This is the condition that there are no $\tilde{q}$ excitations, as can be easily derived from equation (2.17). In section 5.2.1, we identify the two-sphere partition that we obtain as the output of our localization analysis with the partition function of an $\mathcal{N}=(2,2)$ supersymmetric theory, with $\mathrm{U}(K)$ gauge group and

\footnotetext{
${ }^{7}$ Note that the physical parameters are combinations of the form $m_{\psi_{a}}-m_{\psi_{b}}$ and $m_{\psi_{a}}+m_{\tilde{\psi}_{i}}$ and not the masses listed in table 1 , which can be shifted by redefinitions of the vector multiplet scalar.
} 


\begin{tabular}{|c|c|c|c|c|}
\hline Field & $\mathrm{U}(K)$ Representation & Twisted Mass & $R^{(J)}$ & $R^{(R)}$ \\
$X$ & adjoint & 0 & 2 & 0 \\
$\psi_{a}$ & fundamental & $\mu_{a}-\frac{\sum_{b=1}^{N_{c}} \mu_{b}}{C} c_{a}$ & $2-\frac{2 K c_{a}}{C}$ & $\frac{N_{c} c_{a}}{C}$ \\
$\tilde{\psi}_{i}$ & anti-fundamental & $-\mu_{i}+\frac{\sum_{b=1}^{N_{c}} \mu_{b}}{C} c_{i}$ & $\frac{2 K c_{i}}{C}$ & $-\frac{N_{c} c_{i}}{C}$ \\
$\eta_{i, a, r}$ & trivial & $\mu_{a i}$ & $2(r+1)$ & $N_{c} \Delta_{a i}$ \\
\hline
\end{tabular}

Table 2. The spectrum on the worldsheet in cases where the string admits no $\tilde{q}$ excitations.

- 1 chiral multiplet, $X$, in the adjoint representation of $\mathrm{U}(K)$,

- $N_{c}$ chiral multiplets, $\psi_{a}, a=1, \ldots, N_{c}$, in the fundamental representation of $\mathrm{U}(K)$,

- $N_{f}-N_{c}$ chiral multiplets, $\tilde{\psi}_{i}, i=N_{c}+1, \ldots, N_{f}$, in the anti-fundamental representation of $\mathrm{U}(K)$,

- neutral decoupled chiral multiplets, $\eta_{i, a, r}, a=1, \ldots, N_{c}, i=N_{c}+1, \ldots, N_{f}$, $r=0, \ldots, \Delta_{i a} K-1$.

The twisted masses and $R$-charges of the chiral superfields are summarized in table 2 . The FI and theta parameters are given by equations (3.2) and (3.3).

\subsubsection{Comparison with the classical zero-mode analysis}

The GLSM described above gives rise to an NLSM at low-energies. We now compare the spectrum of this NLSM with expectations that are based on the classical string zero-modes analysis of section 2.2 .

The massless adjoint chiral multiplet, as before, consists of the translational moduli and their superpartners. The $N_{c}$ fundamental chiral multiplets, as in the equal charge case, are expected to be associated with excitations of off-diagonal $q_{a}^{b}$ entries, $a, b=1, \ldots, N_{c}$. We will show below that the masses and $R$-charges of the fundamental fields given in table 2 agree with this expectation. Unlike the equal charge case, the existence of these moduli does not follow from a breaking of a global symmetry in the general case.

The decoupled and the anti-fundamental chiral multiplets are associated with the size modes $\rho_{i a}^{(n)}$ of equation (2.17). The division of the size modes into neutral and charged fields can be understood as follows. A scalar $q_{i}^{a}$ gives rise to $k_{a}+\Delta_{i a} K$ size modes. The decoupled modes are the ones that exist for any choice of the partition $\left\{k_{a}\right\}$. These are the $\Delta_{i a} K$ size modes $\rho_{i a}^{(n)}$ with $n=k_{a}+\Delta_{i a} K-1-r$ and $r=0, \ldots, \Delta_{i a} K-1$. Indeed the masses, $R^{(R)}$ - and $R^{(J)}$-charges of these modes are given by $\mu_{a i}, N_{c} \Delta_{a i}$ and $2(r+1)$ respectively, in agreement with the values for $\eta_{i, a, r}$ in table 2 , that were extracted from the localization analysis of section 5 .

The anti-fundamental chiral multiplets are expected to be associated with the sizemodes $\rho_{i a}^{(n)}$ of equation $(2.17)$, with $n=0, \ldots, k_{a}-1$. Since there are exactly $k_{a}$ of these for every $i, a$, they are in one to one correspondence with the zeros of $q_{a}^{a}$, in agreement with the expectation that they transform in the anti-fundamental of $\mathrm{U}(K)$. In order to understand the masses and the $R$-charges of the charged fields, $\psi_{a}$ and $\tilde{\psi}_{i}$ (we use the same 
notation for the multiplet and its bottom component), we need to look at the physical masses and $R$-charges around the vacuum of the worldsheet theory that corresponds to a string solution of the form $(2.20){ }^{8}$

As a warm-up, let us start with the $K=1$ case. One of the $\psi_{a}$ fields must get a vacuum expectation value due to the $\mathrm{D}$-term constraint

$$
\sum_{a=1}^{N_{c}}\left|\psi_{a}\right|^{2}-\sum_{i=N_{c}+1}^{N_{f}}\left|\tilde{\psi}_{i}\right|^{2}=\xi_{2 d} .
$$

Consider the vacuum in which $\psi_{a^{\prime}}$ gets a VEV for some $a^{\prime}$. This vacuum corresponds to the string with windings $k_{a}=\delta_{a, a^{\prime}}$. To cancel the contribution from the mass term to the potential, the gauge multiplet scalar will get a VEV which is equal to the twisted mass of $\psi_{a^{\prime}}$. As a result, the masses of the other charged fields are shifted in this vacuum,

$$
m_{\psi_{a}}=\mu_{a a^{\prime}}, m_{\tilde{\psi}_{i}}=\mu_{a^{\prime} i} .
$$

Similarly, the $R$-charges of the charged fields are shifted in the vacuum with respect to the charges given in table 2 ; the $R$-transformation is accompanied by a $\mathrm{U}(1)$ gauge transformation such that the total transformation leaves $\psi_{a^{\prime}}$ invariant. Thus, the $R$-charges of $\psi_{a}$ and $\tilde{\psi}_{i}$ in this vacuum are

$$
R_{\psi_{a}}^{(J)}=2 \Delta_{a^{\prime} a}, R_{\tilde{\psi}_{i}}^{(J)}=2+2 \Delta_{i a^{\prime}}, R_{\psi_{a}}^{(R)}=N_{c} \Delta_{a a^{\prime}}, R_{\tilde{\psi}_{i}}^{(R)}=N_{c} \Delta_{a^{\prime} i} .
$$

Comparing with equations $(2.9),(2.19)$ and $(2.22)$, we see that the masses and $R^{(R)_{-}}$ charges of $\psi_{a}$ and $\tilde{\psi}_{i}$ coincide with the masses and $R^{(R)}$-charges of the four-dimensional fields $q_{a^{\prime}}^{a}$ and $q_{i}^{a^{\prime}}$ respectively. $R_{\tilde{\psi}_{i}}^{(J)}$ coincide, as expected, with the charges of the size-modes $\rho_{i a^{\prime}}^{(n=0)}$.

The analysis of the $K>1$ vacua and spectrum is more involved. The string configurations of the type (2.20) correspond to solutions of the GLSM $D$-term equations

$$
\sum_{a=1}^{N_{c}} \psi_{a}^{m} \psi_{a, n}^{\dagger}+\left[X, X^{\dagger}\right]_{n}^{m}-\sum_{i=N_{c}+1}^{N_{f}} \tilde{\psi}_{i}^{m}{ }^{\dagger} \tilde{\psi}_{i, n}=\xi_{2 d} \delta_{n}^{m},
$$

together with the vanishing of the mass terms, where the indices $m, n$ in (3.8) are $\mathrm{U}(K)$ color indices. In these vacua, $\tilde{\psi}_{i}=0$. To solve for the vacuum expectation values of the other fields, it will be useful to divide the $K \times K$ matrices $X$ and $\psi_{a} \psi_{a}^{\dagger}$ into $N_{c}$ blocks of sizes $k_{b} \times k_{b}, b=1, \ldots, N_{c}$. We will label the entries of the b'th block by $\alpha_{(b)}, \beta_{(b)}=1, \ldots, k_{b}$. Up to gauge transformations, the solution is given by

$$
\psi_{a}^{\alpha_{(b)}}=\sqrt{k_{a} \xi_{2 d}} \delta^{\alpha_{(a)}, 1} \delta^{a b}, X^{\alpha_{(a)} \beta_{(a)}}=\sqrt{\left(k_{a}-\beta_{(a)}\right) \xi_{2 d}} \delta^{\alpha_{(a)}, \beta_{(a)}+1} .
$$

\footnotetext{
${ }^{8}$ The vacua of the worldsheet theory are in one to one correspondence with $K$-string solutions. In the equal charge case, it was shown that monopoles in the $4 \mathrm{~d}$ theory connect strings with different partitions of $K$ [33]. These monopoles map to kinks in the worldsheet theory that interpolate between the corresponding vacua $[2,12]$. It is easy to check that the agreement between the monopole and kink masses holds also in the cases described in this section.
} 
It is straightforward to verify that this solution solves equation (3.8). The vanishing of the mass terms is satisfied by giving the gauge multiplet scalar the vacuum expectation value

$$
\sigma^{\alpha_{(a)} \beta_{(a)}}=\left(\mu_{a}-\frac{\sum_{b=1}^{N_{c}} \mu_{b}}{C} c_{a}\right) \delta^{\alpha_{(a)} \beta_{(a)}} .
$$

As expected, we find that the GLSM vacua are labeled by integer partitions of $K$.

The $R$-symmetry preserved in such a vacuum is such that the transformation $e^{i\left(\alpha R^{(J)}+(1-\alpha) R^{(R)}\right) \omega_{R}}$ is accompanied by a $\mathrm{U}(K)$ gauge transformation

$$
\psi_{a} \rightarrow U \psi_{a}, \tilde{\psi}_{i} \rightarrow \tilde{\psi}_{i} U^{\dagger}, X \rightarrow U X U^{\dagger}
$$

with

$$
U^{\beta_{(a)}, \gamma_{(a)}}=\delta^{\beta_{(a)}, \gamma_{(a)}} \exp \left(-i\left[\alpha\left(2 \beta_{(a)}-2 K c_{a} / C\right)+(1-\alpha) \frac{N_{c} c_{a}}{C}\right] \omega_{R}\right) .
$$

As a result of the discussion above, the masses and the $R$-charges of the charged fields are shifted such that in the vacuum labeled by the partition $\left\{k_{a}\right\}$ we obtain

$$
\begin{aligned}
& \text { for } \psi_{a}^{\alpha_{(b)}}: \quad m=\mu_{a b}, \quad R^{(R)}=N_{c} \Delta_{a b}, \quad R^{(J)}=2 K \Delta_{b a}+2\left(1-\alpha_{(b)}\right) \text {, } \\
& \text { for } \tilde{\psi}_{i}^{\alpha_{(a)}}: \quad m=\mu_{a i}, \quad R^{(R)}=N_{c} \Delta_{a i}, \quad R^{(J)}=2 K \Delta_{i a}+2 \alpha_{(a)} \text {. }
\end{aligned}
$$

Comparing with equations $(2.9),(2.19)$ and $(2.22)$ we see that the $\tilde{\psi}_{i}$ spectrum matches exactly with the spectrum of the size modes $\rho_{i a}^{(n)}$ with $n=0, \ldots, k_{a}-1$. The $\psi_{a}$ mass and $R^{(R)}$ spectrum, as advertised in the previous section, agrees with the spectrum of $k_{a}+k_{b}$ moduli corresponding to excitations of $q_{a}^{b}$ (accompanied by $q_{b}^{a}$ excitations) for each $a, b$ pair.

\subsection{Strings with $q$ and $\tilde{q}$ excitations}

\subsubsection{Proposal for the worldsheet theory}

We now discuss the most general case for which we have a proposal for a worldsheet theory that is weakly coupled when the four-dimensional theory is weakly coupled. The only restriction on the $\mathrm{U}(1)$ charges is now that the F-term constraints given in equation (2.18) are satisfied trivially and therefore for every $i>N_{c}$ there are either modes coming from $q_{i}$ or modes coming from $\tilde{q}^{i}$, but not both. Without loss of generality, we will assume that for $N_{c}<i \leq N_{q}$, there are only $q_{i}$ modes and for $N_{q}<i \leq N_{f}$ there are only $\tilde{q}^{i}$ modes, for some $N_{c} \leq N_{q} \leq N_{f}$. In terms of the U(1) charges, this assumption means that

$$
\begin{aligned}
\Delta_{i a} \geq 0 & \text { for all } 1 \leq a \leq N_{c}, \quad N_{c}+1 \leq i \leq N_{q}, \\
-\Delta_{i a} \geq 1 & \text { for all } 1 \leq a \leq N_{c}, \quad N_{q}+1 \leq i \leq N_{f} .
\end{aligned}
$$

In section 5.2.2, we identify our proposal for the two-sphere partition function on the string worldsheet with the two-sphere partition function of an $\mathcal{N}=(2,2)$ supersymmetric $\mathrm{U}(K)$ gauge theory with

- 1 chiral multiplet, $X$, in the adjoint representation of $\mathrm{U}(K)$, 


\begin{tabular}{|c|c|c|c|c|}
\hline Field & $\mathrm{U}(K)$ Representation & Twisted Mass & $R^{(J)}$ & $R^{(R)}$ \\
$X$ & adjoint & 0 & 2 & 0 \\
$\psi_{a}$ & fundamental & $\mu_{a}-\frac{\sum_{b b=1}^{N_{c}} \mu_{b}}{C} c_{a}$ & $2-\frac{2 K c_{a}}{C}$ & $\frac{N_{c} c_{a}}{C}$ \\
$\tilde{\psi}_{i}$ & anti-fundamental & $-\mu_{i}+\frac{\sum_{b=1}^{N_{c}} \mu_{b}}{C} c_{i}$ & $\frac{2 K c_{i}}{C}$ & $-\frac{N_{c} c_{i}}{C}$ \\
$\eta_{i, a, r}$ & trivial & $\mu_{a i}$ & $2(r+1)$ & $N_{c} \Delta_{a i}$ \\
$\tilde{\eta}_{i, a, r}$ & trivial & $\mu_{i a}$ & $2(r+1)$ & $2-N_{c} \Delta_{a i}$ \\
\hline
\end{tabular}

Table 3. The spectrum on the worldsheet for strings satisfying (3.14).

- $N_{c}$ chiral multiplets, $\psi_{a}, a=1, \ldots, N_{c}$, in the fundamental representation of $\mathrm{U}(K)$,

- $N_{f}-N_{c}$ chiral multiplets, $\tilde{\psi}_{i}, i=N_{c}+1, \ldots, N_{f}$, in the anti-fundamental representation of $\mathrm{U}(K)$,

- neutral decoupled chiral multiplets, $\eta_{i, a, r}, i=N_{c}+1, \ldots, N_{q}, a=1, \ldots, N_{c}, r=$ $0, \ldots, \Delta_{i a} K-1$,

- neutral chiral multiplets, $\tilde{\eta}_{i, a, r}, i=N_{q}+1, \ldots, N_{f}, a=1, \ldots, N_{c}, r=0, \ldots, \Delta_{a i} K-1$.

The twisted masses and $R$-charges of these multiplets are summarized in table 3 . The FI and theta parameters are given by equations (3.2) and (3.3).

The $\tilde{\eta}$ multiplets can interact with the charged multiplets via the superpotential

$$
W=\sum_{a=1}^{N_{c}} \sum_{i=N_{q}+1}^{N_{f}} \sum_{r=0}^{\Delta_{a i} K-1} \alpha_{r} \tilde{\eta}_{i, a, r} \tilde{\psi}_{i} X^{\Delta_{a i} K-r-1} \psi_{a}
$$

where the color index is suppressed. The coefficients $\alpha_{r}$ cannot be fixed from the localization analysis since the two-sphere partition functions is independent of superpotential couplings $[21,22] .^{9}$ The superpotential is allowed due to the relation

$$
m_{\tilde{\eta}_{i, a, r}}+m_{\tilde{\psi}_{i}}+m_{\psi_{a}}+\left(\Delta_{a i} K-r-1\right) m_{X}=0, R_{\tilde{\eta}_{i, a, r}}+R_{\tilde{\psi}_{i}}+R_{\psi_{a}}+\left(\Delta_{a i} K-r-1\right) R_{X}=2,
$$

which is satisfied for both $R^{(J)}$ and $R^{(R)}$.

\subsubsection{Comparison with the classical zero-mode analysis}

We now describe how the proposal for the worldsheet GLSM fits with the zero-mode analysis. To do this, we will study the spectrum around the GLSM vacuum (3.9) and compare to the classical spectrum of zero modes around the string solution (2.20). The analysis of the first $N_{q}$ hypermultiplets is exactly the same as in the previous section. The only difference comes from the last $N_{f}-N_{q}$ hypermultiplets. For each one of them, the scalar $\tilde{q}_{a}^{i}$ gives rise to $\Delta_{a i} K-k_{a}$ complex modes: $\tilde{\rho}_{i a}^{(n)}, n=0, \ldots, \Delta_{a i} K-k_{a}-1$ (see equation (2.17)).

\footnotetext{
${ }^{9}$ This is true because the massive superalgebra on the sphere preserves a vectorlike $R$-symmetry. If it preserved an axial $R$-symmetry, the sphere partition function would not depend on twisted superpotential couplings.
} 
They are embedded in the chiral multiplets $\tilde{\eta}_{i, a, r}$ with $r=-n+K \Delta_{a i}-k_{a}-1$ (thus, $\left.r=0, \ldots, \Delta_{a i} K-k_{a}-1\right)$. Indeed, the masses and $R$-charges of these size modes, given in equations (2.9), (2.19) and (2.22), agree with those listed in table 3 for $\tilde{\eta}_{i, a, r}$. The remaining fields, $\tilde{\psi}_{i}$ and $\tilde{\eta}_{i, a, r}$ with $r=\Delta_{a i} K-k_{a}, \ldots, \Delta_{a i} K-1$, must vanish around the vacuum (3.9) due to the superpotential constraint equations, as we will show now.

Consider the vacuum (3.9). In this vacuum there exist an $\mathrm{SU}(K)$ invariant operator, positively charged under the $\mathrm{U}(1) \subset \mathrm{U}(K)$, that obtains non-zero expectation value. This operator is obtained by contracting the $\mathrm{U}(K)$ color indices in

$$
\prod_{a=1}^{N_{c}} \prod_{p=0}^{k_{a}-1}\left(X^{p} \cdot \psi_{a}\right)_{n_{a, p}}
$$

using the antisymmetric tensor. The superpotential (3.15) imposes (among many others) the constraints

$$
\frac{\partial W}{\partial \tilde{\eta}_{i, a, K \Delta_{a i}-p-1}}=0 \Rightarrow \tilde{\psi}_{i} X^{p} \psi_{a}=0, \quad a=1, \ldots, N_{c}, \quad p=0, \ldots, k_{a}-1 .
$$

Because (3.17) is not zero, the only solution of (3.18) is $\tilde{\psi}_{i}=0$. After imposing $\tilde{\psi}_{i}=0$, the constraint equations become

$$
\begin{aligned}
\sum_{a=1}^{N_{c}} \psi_{a}^{m} \psi_{a, n}^{\dagger}+\left[X, X^{\dagger}\right]_{n}^{m} & =\xi_{2 d} \delta_{n}^{m}, \\
\sum_{a=1}^{N_{c}} \sum_{r=0}^{\Delta_{a i} K-1} \alpha_{r} \tilde{\eta}_{i, a, r}\left(X^{K \Delta_{a i}-r-1} \cdot \psi_{a}\right)_{n} & =0 .
\end{aligned}
$$

Notice that in the vacuum (3.9), $X^{k_{a}} \psi_{a}=0$. This means that close to the vacuum, the second line in (3.19) is satisfied automatically for $r \leq K \Delta_{a i}-k_{a}-1$. For $r \geq K \Delta_{a i}-k_{a}$, the second line in (3.19) implies that $\tilde{\eta}_{i, a, r}=0$. This shows the agreement between the spectrum of this theory and the spectrum obtained from classical analysis around the string solution. Notice that the coefficient $\alpha_{r}$ cannot be fixed from our analysis. To get the correct spectrum, $\alpha_{r}$ need to be non-vanishing for $r \geq K \Delta_{a i}-K$.

\section{Two-sphere worldsheet partition functions from the four-ellipsoid partition function}

We now move on to the localization analysis of the worldsheet theories. We start this section by reviewing the four-ellipsoid partition function in section 4.1. Then, in section 4.2 we demonstrate, in the $c_{i}=1$ example, the prescription for extracting the worldsheet twosphere partition function from the four-ellipsoid partition function of the original theory. In the next section we will generalize the analysis to general $\mathrm{U}(1)$ charges.

\subsection{The four-ellipsoid partition function}

An $\mathcal{N}=2$ supersymmetric theory that preserves a $\mathrm{U}(1)_{R} \subset \mathrm{SU}(2)_{R} R$-symmetry can be placed on the four-ellipsoid,

$$
\frac{x_{0}^{2}}{r^{2}}+\frac{x_{1}^{2}+x_{2}^{2}}{l^{2}}+\frac{x_{3}^{2}+x_{4}^{2}}{\tilde{l}^{2}}=1,
$$


while preserving an $s u(1 \mid 1)$ superalgebra which contains one supercharge that squares into a linear combination of the two rotations and the $R$-symmetry generator [20],

$$
Q^{2}=\frac{1}{l} J_{1,2}+\frac{1}{\tilde{l}} J_{3,4}+\left(\frac{1}{l}+\frac{1}{\tilde{l}}\right) R
$$

The four-ellipsoid partition function for such a theory has been computed by Hama and Hosomichi using supersymmetric localization [20]. This partition function depends on the dimensionless squashing parameter, $b^{2} \equiv l / \tilde{l}$, on the complexified gauge couplings, and on the dimensionless masses and FI parameters, $\hat{\mu} \equiv \sqrt{l \tilde{l}} \mu, \hat{\xi} \equiv \sqrt{i \tilde{l} \xi} .^{10}$

The four-ellipsoid partition function is given by [20]

$$
Z_{S_{b}^{4}}=\int[d \hat{a}] e^{-S_{\text {cl }}} Z_{1-\text { loop }}^{\text {vec }} Z_{1-\text { loop }}^{\text {hyper }}\left|Z_{\text {inst }}\right|^{2},
$$

where the integral is over the Cartan subalgebra of the gauge group (the integration variables are the rescaled Coulomb branch coordinates, $\hat{a}=\sqrt{l \tilde{l} a}) . S_{\mathrm{cl}}$ is the classical value of the Yang-Mills and Fayet-Illioupulus actions in the localization saddle points,

$$
\begin{aligned}
S_{\mathrm{cl}} & =S_{\mathrm{YM}}+S_{\mathrm{FI}}, \\
S_{\mathrm{YM}} & =\sum_{i} \frac{8 \pi^{2}}{g_{i}^{2}} \operatorname{Tr} \hat{a}_{i}^{2}, \\
S_{\mathrm{FI}} & =-16 i \pi^{2} \sum_{I} \hat{\xi}_{I} \hat{a}_{I},
\end{aligned}
$$

where $g_{i}$ is the Yang-Mills coupling for the gauge factor $G_{i}$ and $\xi_{I}$ and $a_{I}$ are the FI parameters and Coulomb branch parameters for the U(1) factors of the gauge group. The one-loop determinants for the vector multiplets and hypermultiplets are given by

$$
\begin{aligned}
& Z_{1 \text {-loop }}^{\mathrm{vec}}=\prod_{i} \prod_{\alpha \in \Delta_{i}^{+}} \Upsilon_{b}\left(i \hat{a}_{i} \cdot \alpha\right) \Upsilon_{b}\left(-i \hat{a}_{i} \cdot \alpha\right) \\
& Z_{1 \text {-loop }}^{\text {hyper }}=\prod_{R} \prod_{f=1}^{n_{R}} \prod_{w \in P(R)} \Upsilon_{b}\left(i \hat{a} \cdot w+i \hat{\mu}_{f}+\frac{Q}{2}\right)^{-1},
\end{aligned}
$$

where here $i$ runs over the simple factors of the gauge group and $\Delta_{i}^{+}$is the set of positive roots of the simple factor labeled by $i$. The product over $R$ is a product over the irreducible representations of the gauge group, $n_{R}$ is the number of hypermultiplets in the representation $R$, and $\mu_{f}$ denotes the hypermultiplet masses. $P(R)$ is the multiset of weights (with multiplicities) for the representation $R$. The function $\Upsilon_{b}(x)$ is a holomorphic function which is uniquely defined (for $b^{2}$ real and irrational) by the shift relation

$$
\Upsilon_{b}(x+b)=\frac{\Gamma(b x)}{\Gamma(1-b x)} b^{1-2 b x} \Upsilon_{b}(x)
$$

\footnotetext{
${ }^{10}$ From now on, we follow the conventions of [20] for the FI parameter. In these conventions $\xi$ has mass dimension 1. In the case of the round sphere, this parameter is related to the flat space FI parameter by rescaling with the radius. Throughout this work, when a dimension one parameter appears with a hat, it means that it is measured in units of $\sqrt{l \tilde{l}}$.
} 
and the conditions

$$
\begin{aligned}
\Upsilon_{b}(x) & =\Upsilon_{b^{-1}}(x), \\
\Upsilon_{b}\left(\frac{Q}{2}\right) & =1,
\end{aligned}
$$

where $Q=b+b^{-1} \cdot \Upsilon_{b}(x)$ has zeros at

$$
\begin{aligned}
x+m b+n b^{-1} & =0, & & m, n \in \mathbb{N}, \\
Q-x+m b+n b^{-1} & =0, & & m, n \in \mathbb{N} .
\end{aligned}
$$

Here, and throughout this work, $\mathbb{N}$ denotes the set of natural numbers including zero. $Z_{\text {inst }}$ is Nekrasov's instanton partition function in the $\Omega$-background [34], with the equivariant parameters $\epsilon_{1}=l^{-1}, \epsilon_{2}=\tilde{l}^{-1}$.

The asymptotic behavior of the function $\Upsilon_{b}(x)$ in the large $|x|$ limit is of the form $\log \Upsilon_{b}(x)=\frac{1}{2} x(x-Q) \log (x(Q-x))+\left(\frac{3}{2}-\gamma\right) x(Q-x)+\mathcal{O}(\log x)$, where $\gamma$ is the EulerMascheroni constant. As a result, the convergence of the integrals over the Coulomb branch parameters of a $G_{i}$ factor in the gauge group depends on the sign of the $\beta$-function for $g_{i}{ }^{11}$ To allow for string configurations, we need to gauge a $\mathrm{U}(1)$ flavor symmetry. This will make the integral ill-defined due to the U(1) Landau pole. The theory with a gauged $\mathrm{U}(1)$, however, does make sense as a low-energy effective theory, defined at energy scales much smaller than the one set by the Landau pole. We will therefore introduce a cut-off for the Coulomb branch integral, $\hat{\Lambda} \ll e^{\frac{1}{e^{2}}}$, where $e$ is the U(1) gauge coupling.

\subsection{Worldsheet sphere partition functions from the four-ellipsoid partition function in the equal charge case}

In this subsection we will demonstrate, in the case in which $c_{i}=1$ for all $i$, how we extract the two-sphere partition function of the string worldsheet theory from the four-ellipsoid partition function of the full four-dimensional theory. The main result of this section appeared already in [23] (see also [24, 28]). We repeat the full derivation below and add discussions on some of the subtleties in the derivation. In the next section we will generalize the derivation for general $\mathrm{U}(1)$ charges.

We consider an $\mathrm{SU}\left(N_{c}\right)$ gauge theory with $N_{c} \leq N_{f} \leq 2 N_{c}$ hypermultiplets in the fundamental representation. We will gauge the $\mathrm{U}(1)$ flavor symmetry under which all the hypermultiplets have charge 1, and introduce an FI parameter $\xi>0$. The ellipsoid

${ }^{11}$ The asymptotic behavior of the $\Upsilon_{b}$-function implies that the convergence depends on the sign of

$$
2 \sum_{\alpha \in \Delta_{i}^{+}}\left(\alpha \cdot \hat{a}_{i}\right)^{2}-\sum_{R} n_{R} \sum_{w \in P(R)}\left(w \cdot \hat{a}_{i}\right)^{2}=\left(C(\operatorname{adj})-\sum_{R} n_{R} C(R)\right) \hat{a}_{i}^{2}
$$

for simple factors, and that the integral over Coulomb branch parameters for $\mathrm{U}(1)$ factors is always divergent. 
partition function in this case reads

$$
\begin{aligned}
Z_{S_{b}^{4}}= & \left(\prod_{a=1}^{N_{c}} \int_{-\hat{\Lambda}}^{\hat{\Lambda}} d \hat{a}_{a}\right) e^{-\frac{8 \pi^{2}}{g^{2}} \frac{1}{N_{c}} \sum_{a<b}\left(\hat{a}_{a}-\hat{a}_{b}\right)^{2}} e^{-\frac{8 \pi^{2}}{e^{2}} \frac{1}{N_{c}}\left(\sum_{a=1}^{N_{c}} \hat{a}_{a}\right)^{2}} e^{16 i \pi^{2} \hat{\xi} \sum_{a=1}^{N_{c}} \hat{a}_{a}} \\
& \frac{\prod_{a=1}^{N_{c}} \prod_{b \neq a} \Upsilon_{b}\left(i\left(\hat{a}_{a}-\hat{a}_{b}\right)\right)}{\left.\prod_{a=1}^{N_{c}} \prod_{j=1}^{N_{f}} \Upsilon_{b}\left(i\left(\hat{a}_{a}+\hat{\mu}_{j}\right)+\frac{Q}{2}\right)\right)}\left|Z_{\text {inst }}\right|^{2}
\end{aligned}
$$

where $g$ is the Yang-Mills coupling for the $\mathrm{SU}\left(N_{c}\right)$ factor of the gauge group and $e$ is the Yang-Mills coupling for the $\mathrm{U}(1)$ factor. $\mu_{1}, \ldots, \mu_{N_{f}}$ are the hypermultiplet masses. As explained above, we will consider the matrix integral (4.10) with a cut-off $\hat{\Lambda} \ll e^{\frac{1}{e^{2}}}$. We will further assume that $e, g, N_{c} \ll \hat{\Lambda}$, and that

$$
\frac{N_{c} \hat{\Lambda}}{e^{2}}, \frac{N_{c} \hat{\Lambda}}{g^{2}} \ll \hat{\xi} \ll \frac{\hat{\Lambda}^{2}}{e^{2}} b^{-1}, \frac{\hat{\Lambda}^{2}}{g^{2}} b^{-1} .
$$

The assumption $\frac{N_{c} \hat{\Lambda}}{e^{2}}, \frac{N_{c} \hat{\Lambda}}{g^{2}} \ll \hat{\xi}$ allows us to close the integration contours from above, by adding integrations over $\hat{a}_{a}=\hat{\Lambda} e^{i \varphi_{a}}, 0<\varphi_{a}<\pi$. The effect of this modification of the contour is an addition of an $\mathcal{O}\left(\hat{\Lambda} e^{-\frac{\hat{\Lambda}^{2}}{e^{2}}}\right)+\mathcal{O}\left(\hat{\Lambda} e^{-\frac{\hat{\Lambda}^{2}}{g^{2}}}\right)$ contribution to the cutoffed matrix integral. ${ }^{12}$ We neglect such terms in our computation. ${ }^{13}$ The assumption $\hat{\xi} \ll \frac{\hat{\Lambda}^{2}}{e^{2}} b^{-1}, \frac{\hat{\Lambda}^{2}}{g^{2}} b^{-1}$ was added to ensure that the contributions that we want to study are much larger than the contributions that we neglect. More precisely, the condition should be $\hat{\xi} K \ll \frac{\hat{\Lambda}^{2}}{e^{2}} b^{-1}, \frac{\hat{\Lambda}^{2}}{g^{2}} b^{-1}$, where $K$ is the winding number of the string configuration. The interpretation of this condition is that we can only study strings whose energy is much smaller than the cut-off energy. However, any winding number can be placed inside the region of validity of our analysis by choosing $\frac{\hat{\Lambda}^{2}}{e^{2}} b^{-1}, \frac{\hat{\Lambda}^{2}}{g^{2}} b^{-1}$ large enough.

Closing the contours allows us to compute the integrals using Cauchy's theorem, by collecting the residues of the encircled poles. All the poles come from the zeros of the hypermultiplets $\Upsilon_{b}$-function, in the denominator of the one-loop determinant. We assume that the hypermultiplet masses are non-degenerate and therefore all the poles are simple poles.

First, we perform the integration over $\hat{a}_{1}$, summing over the residues of the poles

$$
\hat{a}_{1}=-\hat{\mu}_{l_{1}}+i \frac{Q}{2}+i k_{1} b+i k_{1}^{\prime} b^{-1}, 1 \leq l_{1} \leq N_{f}, k_{1}, k_{1}^{\prime} \in \mathbb{N} .
$$

Then, for each of the residues of the poles in (4.12) we perform the integration over $\hat{a}_{2}$, summing over the residues of the poles,

$$
\hat{a}_{2}=-\hat{\mu}_{l_{2}}+i \frac{Q}{2}+i k_{2} b+i k_{2}^{\prime} b^{-1}, 1 \leq l_{2} \neq l_{1} \leq N_{f}, k_{2}, k_{2}^{\prime} \in \mathbb{N}
$$

\footnotetext{
${ }^{12}$ For small values of $\varphi_{a}$ the smallness of the integrand is ensured by the classical Yang-Mills terms. For finite $\varphi_{a}$ the smallness of the integrand is due to the FI term and the condition $\frac{N_{c} \hat{\Lambda}}{e^{2}}, \frac{N_{c} \hat{\Lambda}}{g^{2}} \ll \hat{\xi}$.

${ }^{13}$ In the limit where $\mathcal{O}\left(\hat{\Lambda} e^{-\frac{\hat{\Lambda}^{2}}{e^{2}}}\right)+\mathcal{O}\left(\hat{\Lambda} e^{-\frac{\hat{\Lambda}^{2}}{g^{2}}}\right)$ are neglected, the precise value of the cut-off is not important, because the integral from $\Lambda$ to $(1+c) \Lambda, c=\mathcal{O}(1)$, is $\mathcal{O}\left(\hat{\Lambda} e^{-\frac{\hat{\Lambda}^{2}}{e^{2}}}\right)+\mathcal{O}\left(\hat{\Lambda} e^{-\frac{\hat{\Lambda}^{2}}{g^{2}}}\right)$.
} 
(note that the residue of the pole (4.12) does not have a pole in $\hat{a}_{2}=-\hat{\mu}_{l_{1}}+i \frac{Q}{2}+i k_{2} b+$ $i k_{2}^{\prime} b^{-1}$ ), and so on. Thus, the non-zero contributions to the matrix integral are given by evaluating the residues of the $N_{c}$ integrals in the simple poles

$$
\hat{a}_{a}=-\hat{\mu}_{l_{a}}+i \frac{Q}{2}+i k_{a} b+i k_{a}^{\prime} b^{-1}, \quad a=1, \ldots, N_{c},
$$

where $k_{a}, k_{a}^{\prime}, l_{a} \in \mathbb{N}, 1 \leq l_{a} \leq N_{f}$, and $l_{a} \neq l_{b}$ for $a \neq b$. The choice of $\left\{l_{a}\right\}$ corresponds to a choice of vacuum in which the $N_{c}$ hypermultiplets labeled by $\left\{l_{a}\right\}$ obtain a vacuum expectation value. Indeed, the real part of $\hat{a}_{a}$ matches the classical VEV of the adjoint scalar given in equation (2.7). Note that we need to include only contributions that satisfy, $\left(\frac{Q}{2}+k_{a} b+k_{a}^{\prime} b^{-1}\right)^{2}+\hat{\mu}_{l_{a}}^{2}<\hat{\Lambda}^{2}$. In addition, as mentioned above, only contributions with

$$
\hat{\xi}\left(\frac{N_{c} Q}{2}+\sum_{a=1}^{N_{c}} k_{a} b+\sum_{a=1}^{N_{c}} k_{a}^{\prime} b^{-1}\right) \ll \frac{\hat{\Lambda}}{e^{2}}, \frac{\hat{\Lambda}}{g^{2}},
$$

are actually significant in the limit we take. This is because the contribution labeled by $\left\{l_{a}, k_{a}, k_{a}^{\prime}\right\}$ comes with a weight

$$
e^{-16 \pi^{2} \hat{\xi}\left(\frac{N_{c} Q}{2}+\sum_{a=1}^{N_{c}} k_{a} b+\sum_{a=1}^{N_{c}} k_{a}^{\prime} b^{-1}\right)-16 i \pi^{2} \hat{\xi} \sum_{a=1}^{N_{c}} \hat{\mu}_{l a} .}
$$

In this work we consider only poles with $k_{a}^{\prime}=0$. We will use the dependence on the squashing parameter $b$ to separate them from the other contributions using the weight factors (4.16). From the point of view of the Higgs branch localization of [23, 24], this amounts to focusing on solutions of the saddle point equations that wrap the squashed two-sphere

$$
\frac{x_{0}^{2}}{r^{2}}+\frac{x_{1}^{2}+x_{2}^{2}}{l^{2}}=1
$$

inside the four-ellipsoid (4.1). Contributions for which both $K=\sum_{a} k_{a}$ and $K^{\prime}=\sum_{a} k_{a}^{\prime}$ are positive correspond to a $K$-string and a $K^{\prime}$-string, wrapping the two-spheres $\frac{x_{0}^{2}}{r^{2}}+$ $\frac{x_{1}^{2}+x_{2}^{2}}{l^{2}}=1$ and $\frac{x_{0}^{2}}{r^{2}}+\frac{x_{3}^{2}+x_{4}^{2}}{\tilde{l}^{2}}=1$ respectively, and intersecting in the two-poles $x_{0}= \pm r$. See $[23,24,28]$.

Using the shift identity

$$
\frac{\Upsilon_{b}(x+n b)}{\Upsilon_{b}(x)}=\prod_{r=0}^{n-1} \frac{\gamma(b(x+r b))}{b^{2 b(x+r b)-1}}, \quad \text { for } n \in \mathbb{N},
$$

where $\gamma(x)=\frac{\Gamma(x)}{\Gamma(1-x)}$, we find a factorized result for the matrix integral,

$$
Z_{S_{b}^{4}}^{(\Lambda)}=e^{-8 \pi^{2} \hat{\xi} N_{c} Q} \sum_{\left\{l_{a}\right\}} e^{-16 i \pi^{2} \hat{\xi} \sum_{a=1}^{N_{c}} \hat{\mu}_{l_{a}}} Z_{\mathrm{vac},\left\{l_{a}\right\}} \sum_{K} e^{-16 \pi^{2} \hat{\xi} K b} Z_{K,\left\{l_{a}\right\}}+\ldots
$$

where

$$
\begin{aligned}
Z_{\mathrm{vac},\left\{l_{a}\right\}}= & \left(\frac{2 \pi}{\Upsilon_{b}^{\prime}(0)}\right)^{N_{c}} e^{-\frac{8 \pi^{2}}{g^{2}} \frac{1}{N_{c}} \sum_{a<b}\left(\hat{\mu}_{l_{b}}-\hat{\mu}_{l_{a}}\right)^{2}} e^{-\frac{8 \pi^{2}}{e^{2}} \frac{1}{N_{c}}\left(-\sum_{a=1}^{N_{c}} \hat{\mu}_{l_{a}}+i N_{c} \frac{Q}{2}\right)^{2}} \\
& \times\left(\prod_{a=1}^{N_{c}} \prod_{j \notin\left\{l_{d}\right\}} \Upsilon_{b}\left(i\left(\hat{\mu}_{j}-\hat{\mu}_{l_{a}}\right)\right)\right)^{-1},
\end{aligned}
$$


and

$$
\begin{aligned}
& Z_{K,\left\{l_{a}\right\}}= \\
& e^{\frac{8 \pi^{2}}{N_{c} e^{2}}\left(K^{2} b^{2}+\left(b^{2}+1\right) K N_{c}+2 i K b \sum_{a=1}^{N_{c}} \hat{\mu}_{l_{a}}\right)} e^{-\frac{8 \pi^{2}}{N_{c} g^{2}}\left(K^{2} b^{2}+2 i b K \sum_{a=1}^{N_{c}} \hat{\mu}_{l_{a}}\right)} \\
& \times b^{N_{f} K\left(1+b^{2}\right)+2 K^{2} b^{2}+2 i b K\left(\sum_{a=1}^{N_{c}} \hat{\mu}_{l_{a}}-\sum_{j \notin\left\{l_{d}\right\}} \hat{\mu}_{j}\right)} \\
& \sum_{\left\{k_{a}\right\} \in \Pi(K)}\left(\left(e^{\frac{8 \pi^{2}}{g^{2}}} b^{N_{f}-2 N_{c}}\right)^{b^{2} \sum_{a=1}^{N_{c}} k_{a}^{2}+2 i b \sum_{a=1}^{N_{c}} k_{a} \hat{\mu}_{l a}}\left[\prod_{a=1}^{N_{c}} \prod_{r=0}^{k_{a}-1} \frac{\prod_{b=1}^{N_{c}} \gamma\left(i b\left(\hat{\mu}_{l_{a}}-\hat{\mu}_{l_{b}}\right)+\left(r-k_{b}\right) b^{2}\right)}{\prod_{j \notin\left\{l_{d}\right\}} \gamma\left(1+(r+1) b^{2}+i b\left(\hat{\mu}_{l_{a}}-\hat{\mu}_{j}\right)\right)}\right]\right. \\
& \left.\quad \times Z_{\text {inst }}^{\text {res }}\left(\mathbb{M}_{i j}=\hat{\mu}_{i}-\hat{\mu}_{j},\left\{k_{a}\right\}, q\right) Z_{\text {inst }}^{\text {res }}\left(\mathbb{M}_{i j}=\hat{\mu}_{i}-\hat{\mu}_{j},\left\{k_{a}\right\}, \bar{q}\right)\right) .
\end{aligned}
$$

Here, $\Pi(K)$ is the set of integer partitions of $K, \sum_{a=1}^{N_{c}} k_{a}=K$. In addition, we denoted

$$
q=e^{2 \pi i \tau_{s u\left(N_{c}\right)}}, \quad \tau_{s u(N)}=\frac{\theta}{2 \pi}+\frac{4 \pi i}{g^{2}} .
$$

The expression for the instanton contributions to the residue, $Z_{\text {inst }}^{\text {res }}\left(\mathbb{M}_{i j},\left\{k_{a}\right\}, q\right)$, can be found in appendix A. ${ }^{14}$ The dots in (4.19) stand for contributions with $K^{\prime} \neq 0$ and $\mathcal{O}\left(\hat{\Lambda} e^{-\frac{\hat{\Lambda}^{2}}{e^{2}}}\right)+\mathcal{O}\left(\hat{\Lambda} e^{-\frac{\hat{\Lambda}^{2}}{g^{2}}}\right)$ corrections.

In (4.19) we expanded the ellipsoid partition function according to the $\xi$ dependence. The interpretation of the different terms in (4.19) is the following. $Z_{\mathrm{vac},\left\{l_{a}\right\}}$ computes the $S_{b}^{4}$ partition function of the light hypermultiplets in the Higgs vacuum labeled by $\left\{l_{a}\right\}$. This partition function is multiplied by a sum over strings with topological charge $K$. Each of these contributions is weighted by a $e^{-16 \pi^{2} \hat{\xi} K b}$ factor, which amounts to the contribution to the energy due to the finite tension of the string. Finally, the string moduli fluctuations are encoded in $Z_{K,\left\{l_{a}\right\}}$, which computes the two-sphere partition function for the low-energy effective theory living on the string worldsheet. In the following, we will check these claims. $e^{-8 \pi^{2} \hat{\xi} N_{c} Q} e^{-16 i \pi^{2} \hat{\xi} \sum_{a=1}^{N_{c}} \hat{\mu}_{l_{a}}} Z_{\mathrm{vac},\left\{l_{a}\right\}}$ is the residue of the integrand in the $\hat{a}_{a}=-\hat{\mu}_{l_{a}}+\frac{i Q}{2}$ poles. In the Higgs vacuum, the vector multiplets eat $N_{c}^{2}$ hypermultiplets and become long massive vector multiplets. Indeed, in $Z_{\mathrm{vac},\left\{l_{a}\right\}}$ the vector multiplet $\Upsilon_{b}$-functions in the numerator are canceled with $N_{c}^{2}$ hypermultiplet $\Upsilon_{b^{-}}$functions. The $N_{c}\left(N_{f}-N_{c}\right) \Upsilon_{b^{-}}$ functions left in $Z_{\mathrm{vac},\left\{l_{a}\right\}}$ correspond to the $N_{f}-N_{c}$ light hypermultiplets left in the Higgs vacuum, with complex masses $\hat{\mu}_{j}-\hat{\mu}_{l_{a}}+\frac{i Q}{2}$. Like the vacuum expectation values for the adjoint scalars, these masses are shifted with respect to the flat space masses by $\frac{i Q}{2}$. This shift is related to the fact that the $R$-symmetry preserved in the vacuum is shifted with respect to the Cartan of $\mathrm{SU}(2)_{R}$, as explained above equation (2.11). See the next section for more details.

Let us now move on to the interpretation of $Z_{K,\left\{l_{a}\right\}}$ as the two-sphere partition function of the worldsheet theory. The strings described by $Z_{K,\left\{l_{a}\right\}}$ wrap the squashed two-sphere whose embedding in the four-ellipsoid (4.1) is given by equation (4.17). An $\mathcal{N}=(2,2)$ supersymmetric theory with a vectorlike $\mathrm{U}(1) R$-symmetry can be placed on the round

\footnotetext{
${ }^{14}$ The expression in appendix $\mathrm{A}$ is written for $l_{a}=a$.
} 
two-sphere $(l=r)$ while preserving an $s u(2 \mid 1)$ superalgebra $[21,22] .{ }^{15}$ One can squash the sphere while preserving an $s u(1 \mid 1) \subset s u(2 \mid 1)$ subalgebra. The squashed two-sphere partition function does not depend on the squashing parameter $\frac{l}{r}$ [35], and is equal to the partition function of the round two-sphere with radius $l$, which was computed in $[21,22]$ using localization techniques.

For an $\mathcal{N}=(2,2)$ supersymmetric theory with gauge group $\mathrm{U}(K), N_{c}$ chiral multiplets in the fundamental representation, $N_{f}-N_{c}$ chiral multiplets in the anti-fundamental representation and one chiral multiplet in the adjoint representation, the Higgs branch representation of the two-sphere partition function is [36]

$$
\begin{aligned}
Z_{S^{2}, K}^{\mathrm{SQCDA}}=\sum_{\left\{k_{a}\right\} \in \Pi(K)}\left[(z \bar{z})^{-i\left(\sum_{a=1}^{N_{c}} k_{a} M_{a}+\frac{M_{X}}{2}\left(\sum_{a=1}^{N_{c}} k_{a}^{2}-K\right)\right)} \times\right. & \\
& \left.\frac{\prod_{b=1}^{N_{c}} \prod_{a=1}^{N_{c}} \prod_{r=0}^{k_{a}-1} \gamma\left(i\left(M_{a}-M_{b}\right)+i\left(r-k_{b}\right) M_{X}\right)}{\prod_{j=1}^{N_{f}-N_{c}} \prod_{a=1}^{N_{c}} \prod_{r=0}^{k_{a}-1} \gamma\left(1+i M_{a}+i \tilde{M}_{j}+i r M_{X}\right)} Z_{\left\{k_{a}\right\}}^{\mathrm{vort}}(z) Z_{\left\{k_{a}\right\}}^{\mathrm{vort}}(\bar{z})\right],
\end{aligned}
$$

where $z=\exp \left(-2 \pi \xi_{2 d}+i \theta_{2 d}\right)$, with $\xi_{2 d}$ the FI parameter of the GLSM and $\theta_{2 d}$ the theta-parameter. The twisted masses and $R$-charges of the chiral multiplets are combined in (4.23) into the dimensionless complexified twisted masses, $M=l m+\frac{i}{2} R$, where $m$ denotes the twisted mass of the multiplet, $R$ its $R$-charge and $l$ is the radius of the twosphere (which, in our case is equal to one of the radii of the ellipsoid). In the expression above, $M_{a}$ are the complexified twisted masses of the fundamental chiral multiplets, $\tilde{M}_{j}$ are the complexified twisted masses of the anti-fundamental chiral multiplets and $M_{X}$ is the complexified twisted mass of the adjoint chiral multiplet. The function $Z_{\left\{k_{a}\right\}}^{\text {vort }}$, describing the non-perturbative contributions, can be found in appendix A.

The dictionary between the four-dimensional and two-dimensional parameters that can be obtained by comparing (4.21) with (4.23), as we will explain below, is

$$
\begin{aligned}
z & =b^{2 N_{c}-N_{f}} q, \\
M_{a}-M_{b} & =b\left(\hat{\mu}_{l_{a}}-\hat{\mu}_{l_{b}}\right), \\
M_{a}+\tilde{M}_{j} & =b\left(\hat{\mu}_{l_{a}}-\hat{\mu}_{n_{j}}\right)-i b^{2}, \\
M_{X} & =-i b^{2}
\end{aligned}
$$

where $\left\{n_{1}, n_{2}, \ldots, n_{N_{f}-N_{c}}\right\}=\left\{1, \ldots, N_{f}\right\} \backslash\left\{l_{a} \mid a=1, \ldots, N_{c}\right\}$. In terms of the masses and $R$-charges, the dictionary reads

$$
\begin{aligned}
m_{a}-m_{b} & =\mu_{l_{a}}-\mu_{l_{b}}, & R_{a}-R_{b} & =0, \\
m_{a}+\tilde{m}_{j} & =\mu_{l_{a}}-\mu_{n_{j}}, & R_{a}+\tilde{R}_{n_{j}} & =-2 b^{2}, \\
m_{X} & =0, & R_{X} & =-2 b^{2} .
\end{aligned}
$$

\footnotetext{
${ }^{15}$ Similarly, an $\mathcal{N}=(2,2)$ supersymmetric theory with an axial $\mathrm{U}(1) R$-symmetry can be placed on the round sphere while preserving a different $s u(2 \mid 1)$ superalgebra, that contains the axial $R$-symmetry instead of the vector $R$-symmetry.
} 
Before showing agreement between (4.21) and (4.23) under the dictionary (4.24), let us make a few comments about the map of the parameters. The $s u(1 \mid 1)$ superalgebra on the squashed two-sphere is inherited from the $s u(1 \mid 1)$ superalgebra on the four-ellipsoid in the following way

$$
Q^{2}=\frac{1}{l} J_{1,2}+\frac{1}{\tilde{l}} J_{3,4}+\left(\frac{1}{l}+\frac{1}{\tilde{l}}\right) R_{4 d}=\frac{1}{l}\left(J_{1,2}-b^{2} J_{4,3}+\left(1+b^{2}\right) R_{4 d}\right)=\frac{1}{l}\left(J_{1,2}+R_{2 d}\right) .
$$

In other words, the $R$-symmetry that appears in the $s u(1 \mid 1)$ algebra on the two-sphere is a combination the four-dimensional $R$-symmetry and rotations in the transverse plane with coefficients $b^{2}+1$ and $-b^{2}$ respectively. We denote the corresponding worldsheet $R$-symmetries by $R^{(R)}$ and $R^{(J)}$. The dependence on $b^{2}$ allows us to extract the $R^{(R)}$ - and $R^{(J)}$-charges of the chiral multiplets from the dictionaries we find. In section 3 we compared the $R^{(R)}$ - and $R^{(J)}$-charges of the worldsheet fields that we read from the localization analysis with the expected charges based on a classical zero-mode analysis.

The $b^{2 N_{f}-N_{c}}$ factor in the map (4.24) can be understood in the following way. The couplings $g^{2}$ and $\xi_{2 d}$ that appear in the localization formulas are given at the scales $\frac{1}{\sqrt{i \tilde{l}}}$ and $\frac{1}{l}$ respectively. Thus, the correct way to read the map we obtained is

$$
\frac{4 \pi}{g^{2}}\left(\frac{1}{\sqrt{l i}}\right)-\xi_{2 d}\left(\frac{1}{l}\right)=\frac{1}{2 \pi}\left(2 N_{c}-N_{f}\right) \log b .
$$

When the couplings are measured at the same scale, the $\log b$ correction disappears, since

$$
\begin{aligned}
\frac{4 \pi}{g^{2}}(\mu)-\xi_{2 d}(\mu)= & {\left[\frac{4 \pi}{g^{2}}\left(\frac{1}{\sqrt{l \tilde{l}}}\right)+\frac{1}{2 \pi}\left(2 N_{c}-N_{f}\right) \log (\mu \sqrt{l \tilde{l}})\right] } \\
& -\left[\xi_{2 d}\left(\frac{1}{l}\right)+\frac{1}{2 \pi}\left(2 N_{c}-N_{f}\right) \log (\mu l)\right]=0 .
\end{aligned}
$$

Using the dictionary (4.24), equation (4.21) is mapped to equation (4.23), up to a multiplicative factor

$$
\begin{aligned}
\frac{Z_{K,\left\{l_{a}\right\}}}{Z_{S^{2}, K}^{\mathrm{SQCDA}}}= & e^{-4 \pi i \xi_{2 d} \frac{K}{N_{c}} \sum_{a=1}^{N_{c}} M_{a}} e^{-2 \pi K \xi_{2 d} b^{2}\left(-1+\frac{K}{N_{c}}\right)} \\
& b^{N_{f} K\left(1-b^{2}\right)+2 K b^{2} N_{c}+2 K^{2} b^{2}+2 i K\left(\sum_{a=1}^{N_{c}} M_{a}+\sum_{j=1}^{N_{f}-N_{c}} \tilde{M}_{j}\right)+\frac{N_{f}-2 N_{c}}{N_{c}}\left(K^{2} b^{2}+2 i K \sum_{a=1}^{N_{c}} M_{a}\right)} \\
& e^{\frac{8 \pi^{2}}{N_{c} e^{2}}\left(K^{2} b^{2}+\left(b^{2}+1\right) K N_{c}+2 i K b \sum_{a=1}^{N_{c}} \hat{\mu}_{l_{a}}\right)} .
\end{aligned}
$$

For the comparison of the non-perturbative contributions, see appendix A. We would like to argue that the multiplicative factor (4.29) is not universal, in the sense that it can be removed with an appropriate choice of regularization scheme.

Two two-sphere partition functions that were obtained in two different regularization schemes may differ by local counterterms. A useful way to classify the allowed counterterms, assuming that the two regularization schemes preserve the $s u(2 \mid 1)$ superalgebra on 
the sphere ${ }^{16}$ is to promote the couplings to fields transforming in representations of supergravity. The regularization ambiguities of the sphere free-energy, $\log Z_{S^{2}}$, are then given by supergravity Lagrangians composed out of these fields, evaluated at the sphere constant background.

The complexified FI parameter, $t=i \xi_{2 d}+\frac{\theta_{2 d}}{2 \pi}$, sits in the bottom component of a background twisted chiral multiplet with $R$-charge zero. A supergravity Lagrangian that evaluates to $f(t)+\bar{f}(\bar{t})$ in the sphere background was constructed in [37], thus showing that $Z_{S^{2}}$ has ambiguity of the form

$$
Z_{S^{2}} \sim f(z) \bar{f}(\bar{z}) Z_{S^{2}}
$$

for any holomorphic function $f$.

The masses $m_{a}$ and $\tilde{m}_{j}$ also need to be promoted to supergravity multiplets. The sum of the masses $\sum_{a=1}^{N_{c}} m_{a}$ sits in a real scalar component of a $\mathrm{U}(1)$ vector multiplet, which corresponds to weakly gauging the flavor symmetry under which all the fundamental fields have charge 1 and all the anti-fundamental fields have charge zero. The same supergravity Lagrangian that produces the FI terms on the sphere can be used to write a local counterterm that, when evaluated in the sphere supersymmetric background, is proportional to $i l \xi_{2 d}\langle\sigma\rangle$, where $\langle\sigma\rangle$ is any combination of the masses that corresponds to a conserved $\mathrm{U}(1)$ flavor symmetry. Thus, the two-sphere partition function has ambiguity of the form

$$
Z_{S^{2}} \sim(z \bar{z})^{i l\langle\sigma\rangle} Z_{S^{2}} .
$$

Taking $\langle\sigma\rangle \propto \sum_{a=1}^{N_{c}} m_{a}$, one can absorb the $\exp \left(-4 \pi i \xi_{2 d} \frac{K}{N_{c}} l \sum_{a=1}^{N_{c}} m_{a}\right)$ term in the first line of (4.29). See also the discussion in [36].

The dependence of (4.29) on $b^{2}$ needs to be realized through a supersymmetric counterterm that contains the $R$-charges of the fields. Thus, the $R$-charges also need to be embedded in a supergravity representation. However, this analysis appears to be more subtle. We leave this problem for the future; we will not try to explain the dependence of equation (4.29) on $b^{2}$ and the imaginary parts of the complexified masses.

Finally, the last term in (4.29),

$$
e^{\frac{8 \pi^{2}}{N_{c} e^{2}}\left(K^{2} b^{2}+\left(b^{2}+1\right) K N_{c}+2 i K b \sum_{a=1}^{N_{C}} \hat{\mu}_{l_{a}}\right)},
$$

depends on two parameters of the $4 \mathrm{~d}$ theory, $e^{-2}$ and $\sum_{a=1}^{N_{c}} \mu_{l_{a}}$, that have no interpretation in the $2 \mathrm{~d}$ theory. To argue that (4.32) is a trivial contribution to the two-sphere partition function, we formally map these parameters to two additional parameters - an FI term and a mass parameter that correspond to a U(1) symmetry in a decoupled theory - and notice that this decoupled theory is in fact trivial, as (4.32) is interpreted as a supersymmetric counterterm using (4.30) and (4.31).

To conclude, the expression (4.21) that was extracted from the four-ellipsoid partition function computes the two-sphere partition function of the GLSM described in section 3.1, whose low-energy limit has been conjectured in $[3,6]$ to describe the worldsheet theory on the $K$-string.

\footnotetext{
${ }^{16}$ Note that any $s u(2 \mid 1)$ supersymmetric counterterm is also $s u(1 \mid 1)$ supersymmetric.
} 


\section{General U(1) charges}

\subsection{The worldsheet two-sphere partition function for a general string}

In this section we generalize the analysis of the previous section by allowing different $\mathrm{U}(1)$ charges for different hypermultiplets. As before, the $\mathrm{U}(1)$ charges will be denoted by $c_{i}$, $i=1, \ldots, N_{f}$.

The four-ellipsoid partition function is now given by the matrix integral

$$
\begin{aligned}
Z_{S_{b}^{4}}= & \int\left(\prod_{a=1}^{N_{c-1}} d\left(w_{a} \cdot \hat{a}\right)\right) d \hat{a}^{\prime} e^{-\frac{16 \pi^{2}}{g^{2}} \hat{a} \cdot \hat{a}-\frac{8 \pi^{2}}{e^{2}} N_{c} \hat{a}^{\prime 2}+16 i \pi^{2} N_{c} \hat{\xi} \hat{a}^{\prime}} \\
& \times \frac{\prod_{a \neq b} \Upsilon_{b}\left(i w_{a} \cdot \hat{a}-i w_{b} \cdot \hat{a}\right)}{\prod_{a=1}^{N_{c}} \prod_{i=1}^{N_{f}} \Upsilon_{b}\left(i w_{a} \cdot \hat{a}+i c_{i} \hat{a}^{\prime}+i \hat{\mu}_{i}+\frac{Q}{2}\right)}\left|Z_{\text {inst }}\right|^{2},
\end{aligned}
$$

where $w_{a}$ are the weights in the fundamental representation of $\operatorname{SU}\left(N_{c}\right)\left(\sum_{a=1}^{N_{c}} w_{a}=0\right)$. The instanton partition function is given by equation (A.1) of appendix A.

The denominator $\Upsilon_{b}$-functions vanish at

$$
\begin{aligned}
& i w_{a} \cdot \hat{a}+i c_{j} \hat{a}^{\prime}+i \hat{\mu}_{j}+\frac{Q}{2}+k_{a, j} b+k_{a, j}^{\prime} b^{-1}=0, a=1, \ldots, N_{c}, j=1, \ldots, N_{f}, k_{a, j}, k_{a, j}^{\prime} \in \mathbb{N} \\
& i w_{a} \cdot \hat{a}+i c_{j} \hat{a}^{\prime}+i \hat{\mu}_{j}-\frac{Q}{2}-k_{a, j} b-k_{a, j}^{\prime} b^{-1}=0, a=1, \ldots, N_{c}, j=1, \ldots, N_{f}, k_{a, j}, k_{a, j}^{\prime} \in \mathbb{N}
\end{aligned}
$$

As in the previous section, we introduce a UV cut-off for the matrix integral and perform the integrals step by step. In the first step we integrate over $\hat{a}^{\prime}$, closing the contour from above and picking up the residues of the encircled poles. For each one of the $\hat{a}^{\prime}$-poles we then continue with the next integrals. For the poles of $\hat{a}^{\prime}$ corresponding to color and flavor indices $\hat{a}$ and $j$ in the hypermultiplet one-loop determinant, we perform in the next step the integration over $w_{a} \cdot \hat{a}$, closing the contour from above if $c_{j}>0$ or from below if $c_{j}<0$. (There are no poles for $\hat{a}^{\prime}$ corresponding to $c_{j}=0$.) We continue in a similar fashion. After the matrix integral has been computed, we expand the result according to the $\hat{\xi}$ dependence and attempt to identify the bulk and the string contributions as in the previous section.

If $N_{c}>2$ and not all the charges are equal, there are contributions to the four-ellipsoid partition function for which we are not able to close all the integration contours. These are obtained by picking poles with the same color index in the first two integrations, one of the form (5.2) and one of the form (5.3). After the first two integrations, the FI parameter will not multiply any of the $N_{c}-2$ integration variables we are left with, and we will not be able to close the contours. We will therefore stay with an $N_{c}-2$ dimensional matrix integral. These contributions correspond to the mesonic vacua and the mesonic strings mentioned in section 2, which have $N_{c}-2$ flat Coulomb branch directions. For $N_{c}=2$, the mesonic vacua and strings are essentially the same as the baryonic vacua and strings due to the fact that the fundamental representation of $\mathrm{SU}(2)$ is pseudo-real. We will discuss mesonic strings in an $N_{c}=2$ example in section 6.2.3. 
The other contributions correspond to the baryonic vacua and strings. For these contributions, we are able to close all the contours and obtain the contribution as a residue of $N_{c}$ poles corresponding to $N_{c}$ different color indices. The residue of a pole with flavor index $j$ will not have poles in the remaining integration variables with the same flavor index. Therefore, the non-vanishing contributions pick $N_{c}$ out of the $N_{f}$ hypermultiplets. One can show that it is enough to consider only contributions that come from picking $N_{c}$ poles of the form (5.2) and contributions that come from picking $N_{c}$ poles of the form (5.3), as mixed combinations of poles will cancel among themselves. Therefore, we need to sum over the combination of poles that solve

$i w_{a} \cdot \hat{a}+i c_{l_{a}} \hat{a}^{\prime}+i \hat{\mu}_{l_{a}}+\frac{Q}{2}+k_{a} b+k_{a}^{\prime} b^{-1}=0, a=1, \ldots, N_{c}, \quad\left\{l_{1}, \ldots, l_{N_{f}}\right\} \subset\left\{1, \ldots, N_{f}\right\}, \quad k_{a}, k_{a}^{\prime} \in \mathbb{N}$,

with $\sum_{a=1}^{N_{c}} c_{l_{a}}>0$ and combination of poles that solve

$i w_{a} \cdot \hat{a}+i c_{l_{a}} \hat{a}^{\prime}+i \hat{\mu}_{l_{a}}-\frac{Q}{2}-k_{a} b-k_{a}^{\prime} b^{-1}=0, a=1, \ldots, N_{c}, \quad\left\{l_{1}, \ldots, l_{N_{f}}\right\} \subset\left\{1, \ldots, N_{f}\right\}, \quad k_{a}, k_{a}^{\prime} \in \mathbb{N}$,

with $\sum_{a=1}^{N_{c}} c_{l_{a}}<0$.

Thus, we obtain the four-ellipsoid partition function as a sum over mesonic and baryonic contributions,

$$
\begin{aligned}
Z_{S_{b}^{4}}^{(\Lambda)}= & \sum_{\left\{l_{a}\right\}} Z_{\mathrm{vac},\left\{l_{a}\right\}}^{B} e^{-8 \pi^{2} \hat{\xi} N_{c}^{2} Q /\left|C_{\left\{l_{a}\right\}}\right|} e^{-16 i \pi^{2} N_{c} \hat{\xi} \sum_{a=1}^{N_{c}} \hat{\mu}_{l_{a}} / C_{\left\{l_{a}\right\}}} \\
& \times \sum_{K, K^{\prime}} e^{-16 \pi^{2} N_{c} \hat{\xi}\left(K b+K^{\prime} b^{-1}\right) /\left|C_{\left\{l_{a}\right\}}\right|} Z_{K, K^{\prime}\left\{l_{a}\right\}}^{B} \\
& +\sum_{i, j, c_{i} \neq c_{j}} e^{-16 i \pi^{2} N_{c} \hat{\xi} \frac{\hat{\mu}_{i}-\hat{\mu}_{j}}{c_{i}-c_{j}}} \sum_{K, K^{\prime}} e^{-\frac{16 \pi^{2} N_{c} \hat{\xi}}{\left|c_{i}-c_{j}\right|}\left(K b+K^{\prime} b^{-1}\right)} Z_{K, K^{\prime}, i, j}^{M},
\end{aligned}
$$

where $C_{\left\{l_{a}\right\}}=\sum_{a=1}^{N_{c}} c_{l_{a}}$. The first line represents the baryonic vacua and strings and the second line represents the contributions of the mesonic vacua and strings. $Z_{\mathrm{vac},\left\{l_{a}\right\}}^{B}$, $Z_{K, K^{\prime}\left\{l_{a}\right\}}^{B}$ and $Z_{K, K^{\prime}, i, j}^{M}$ are independent of $\hat{\xi}$.

We will focus on the baryonic contributions. To simplify the notation, let us take $\left\{l_{a}\right\}=\left\{1,2, \ldots, N_{c}\right\}$, and assume that $C=C_{\left\{l_{a}\right\}=\left\{1,2, \ldots, N_{c}\right\}}=\sum_{a=1}^{N_{c}} c_{a}>0$ (i.e. we focus on poles of the form (5.4)). The vacuum contributions are then given by

$$
\begin{aligned}
Z_{\mathrm{vac},\left\{1,2, \ldots, N_{c}\right\}}^{B} \\
=N_{c} !\left(\frac{2 \pi}{\Upsilon_{b}^{\prime}(0)}\right)^{N_{c}} e^{\frac{8 \pi^{2}}{g^{2}} \sum_{a=1}^{N_{c}}\left(-i \hat{\mu}_{a}+i \frac{c_{a}}{C} \sum_{b=1}^{N_{c}} \hat{\mu}_{b}+\left(\frac{c_{a}}{C} N_{c}-1\right) \frac{Q}{2}\right)^{2}} e^{-\frac{8 \pi^{2}}{e^{2}} \frac{N_{c}}{C^{2}}\left(-\sum_{a=1}^{N_{c}} \hat{\mu}_{a}+i N_{c} \frac{Q}{2}\right)^{2}} \\
\prod_{a=1}^{N_{c}} \prod_{i=N_{c}+1}^{N_{f}} \Upsilon_{b}\left(i\left(\hat{\mu}_{i}-\hat{\mu}_{a}\right)-i \Delta_{i a} \sum_{b=1}^{N_{c}} \hat{\mu}_{b}-\Delta_{i a} N_{c} \frac{Q}{2}\right)^{-1}
\end{aligned}
$$

As before, $Z_{\mathrm{vac},\left\{1, \ldots, N_{c}\right\}}^{B}$ is interpreted as the four-ellipsoid partition function of the light hypermultiplets in the vacuum (2.7). Indeed, the arguments of the $\Upsilon_{b}$-functions imply a 
complexified mass spectrum on the ellipsoid; $\mu_{i}-\mu_{a}+\Delta_{a i} \sum_{b=1}^{N_{c}} \mu_{b}+\frac{i}{2}\left(\frac{1}{l}+\frac{1}{l}\right)\left(\Delta_{i a} N_{c}+1\right)$. The real part gives the flat space mass spectrum, which agrees exactly with equation (2.9). The imaginary shift in the mass is proportional to the shift in the $R$-charge of the multiplet in the vacuum - see the discussion above equation (2.11). This is related to the fact that a background $R$-current needs to be turned on in order to preserve supersymmetry on the ellipsoid [20]. Equation (5.7) needs to be compared to the partition function of a theory that has been placed on the ellipsoid using the $R$-symmetry preserved by the vacuum (2.7).

Next, the function $Z_{K, K^{\prime},\left\{1,2, \ldots, N_{c}\right\}}^{B}$ is given by

$$
\begin{aligned}
& Z_{K, K^{\prime},\left\{1,2, \ldots, N_{c}\right\}}^{B}=e^{-\frac{8 \pi^{2}}{e^{2}} \frac{N_{c}}{C^{2}}\left[2 i\left(-\sum_{a=1}^{N_{c}} \hat{\mu}_{a}+i N_{c} \frac{Q}{2}\right)\left(K b+K^{\prime} b^{-1}\right)-\left(K b+K^{\prime} b^{-1}\right)^{2}\right]} \\
& \sum_{\left\{k_{a}\right\} \in \Pi(K)} \sum_{\left\{k_{a}^{\prime}\right\} \in \Pi\left(K^{\prime}\right)}\left\{\left.(-1)^{\sum_{a=1}^{N_{c}} k_{a} k_{a}^{\prime}}\left|Z_{\text {inst }}\right|^{2}\right|_{i w_{a} \cdot \hat{a}+i c_{l_{a}} \hat{a}^{\prime}+i \hat{\mu}_{l_{a}}+\frac{Q}{2}+k_{a} b+k_{a}^{\prime} b^{-1}=0}\right. \\
& e^{\frac{8 \pi^{2}}{g^{2}} \sum_{a=1}^{N_{c}}\left[2\left(-i \hat{\mu}_{a}+i \frac{c_{a}}{C} \sum_{b=1}^{N_{c}} \hat{\mu}_{b}+\left(\frac{c_{a}}{C} N_{c}-1\right) \frac{Q}{2}\right)\left(\frac{c_{a}}{C}\left(K b+K^{\prime} b^{-1}\right)-k_{a} b-k_{a}^{\prime} b^{-1}\right)+\left(\frac{c_{a}}{C}\left(K b+K^{\prime} b^{-1}\right)-k_{a} b-k_{a}^{\prime} b^{-1}\right)^{2}\right]} \\
& \left(\prod_{a=1}^{N_{c}} \prod_{r=0}^{k_{a}-1} \frac{\gamma\left(-(r+1) b^{2}\right)}{b^{-2(1+r) b^{2}-1}}\right)\left(\prod_{a=1}^{N_{c}} \prod_{s=0}^{k_{a}^{\prime}-1} \frac{\gamma\left(-(s+1) b^{-2}\right)}{b^{2(1+s) b^{-2}+1}}\right)\left(\prod_{a=1}^{N_{c}} \prod_{r=0}^{k_{a}-1} \prod_{s=0}^{k_{a}^{\prime}-1}\left(-(r+1) b-(s+1) b^{-1}\right)^{-2}\right) \\
& \prod_{a=1}^{N_{c}} \prod_{i=N_{c}+1}^{N_{f}} \frac{\Upsilon_{b}\left(i\left(\hat{\mu}_{i}-\hat{\mu}_{a}\right)-i \Delta_{i a} \sum_{b=1}^{N_{c}} \hat{\mu}_{b}-\Delta_{i a} N_{c} \frac{Q}{2}\right)}{\Upsilon_{b}\left(i\left(\hat{\mu}_{i}-\hat{\mu}_{a}\right)-i \Delta_{i a} \sum_{b=1}^{N_{c}} \hat{\mu}_{b}-\Delta_{i a} N_{c} \frac{Q}{2}-k_{a} b-k_{a}^{\prime} b^{-1}-\Delta_{i a}\left(K b+K^{\prime} b^{-1}\right)\right)} \\
& \left.\prod_{a=1}^{N_{c}} \prod_{b \neq a} \frac{\Upsilon_{b}\left(i\left(\hat{\mu}_{b}-\hat{\mu}_{a}\right)+i \Delta_{a b} \sum_{a=1}^{N_{c}} \hat{\mu}_{a}+\Delta_{a b} N_{c} \frac{Q}{2}+\left(k_{b}-k_{a}\right) b+\left(k_{b}^{\prime}-k_{a}^{\prime}\right) b^{-1}+\Delta_{a b}\left(K b+K^{\prime} b^{-1}\right)\right)}{\Upsilon_{b}\left(i\left(\hat{\mu}_{b}-\hat{\mu}_{a}\right)+i \Delta_{a b} \sum_{c=1}^{N_{c}} \hat{\mu}_{c}+\Delta_{a b} N_{c} \frac{Q}{2}-k_{a} b-k_{a}^{\prime} b^{-1}+\Delta_{a b}\left(K b+K^{\prime} b^{-1}\right)\right)}\right\} .
\end{aligned}
$$

If $N_{f}=N_{c}$ we can use the shift identity,

$$
\begin{aligned}
& \frac{\Upsilon_{b}\left(x+n b+k b^{-1}\right)}{\Upsilon_{b}(x)} \\
& =(-1)^{n k}\left(\prod_{r=0}^{n-1} \frac{\gamma(b(x+r b))}{b^{2 b(x+r b)-1}}\right)\left(\prod_{s=0}^{k-1} \frac{\gamma\left(b^{-1}\left(x+s b^{-1}\right)\right)}{\left(b^{-1}\right)^{2 b^{-1}\left(x+s b^{-1}\right)-1}}\right)\left(\prod_{r=0}^{n-1} \prod_{s=0}^{k-1}\left(x+r b+s b^{-1}\right)^{2}\right),
\end{aligned}
$$

applicable for $n, k \in \mathbb{N}$, to rewrite $Z_{K, K^{\prime}}^{B}$ in terms of $\Gamma$-functions, the "building-blocks" of the two-sphere partition function. When $N_{f}>N_{c}$, however, (5.9) will be applicable only if

$$
\Delta_{i j} K, \Delta_{i j} K^{\prime} \in \mathbb{Z}, \text { for all } i, j .
$$

Indeed, when $N_{f}>N_{c}$ the theory is not gapped - there are light fields in the bulk that in general interact with the string moduli. The contribution of a $K$-string satisfying the condition (5.10) to the four-ellipsoid partition function factorizes to contributions that look like a four-ellipsoid partition function multiplying two-sphere partition function. This suggests that when the condition (5.10) is satisfied, the bulk and string moduli decouple at low energies. Remarkably, this is exactly the condition that we found in a classical analysis of the interaction terms in [1]. 
As explained in the introduction, the sphere partition function does not capture all the information encoded in the theory. Interpreting the factorization that we observe as a clear sign for $4 \mathrm{~d}-2 \mathrm{~d}$ decoupling is therefore subtle, as some $4 \mathrm{~d}-2 \mathrm{~d}$ interaction terms can be invisible to the sphere partition function. For example, in the case discussed in section 3.3 (and section 5.2.2), one can write down a $2 \mathrm{~d}$ superpotential as in [36], that couples $2 \mathrm{~d}$ chiral multiplets to a $4 \mathrm{~d}$ hypermultiplet restricted to the string (or, more precisely, to an $\mathcal{N}=(2,2)$ chiral multiplet inside the $4 \mathrm{~d}$ hypermultiplet). The superpotential takes the form $\left.\tilde{q}_{a}^{j}\right|_{2 d} \tilde{\psi}_{j} X^{K \Delta_{a j}} \psi_{a}$, and can be written only for $a, j$ such that $\Delta_{a j} \geq 0$. Here $\psi, \tilde{\psi}$ and $X$ are the $2 \mathrm{~d}$ fields defined in section 3 , and $\left.\tilde{q}_{a}^{j}\right|_{2 d}$ is the appropriate $\mathcal{N}=(2,2)$ chiral multiplet that contains the restriction of the $4 \mathrm{~d}$ scalar $\tilde{q}_{a}^{j}$ to the string. ${ }^{17}$ The localization analysis cannot tell us anything about the couplings of such terms. The classical arguments of [1], however, suggest that $4 \mathrm{~d}-2 \mathrm{~d}$ interactions are suppressed at energies $E \ll m_{W}$, where $m_{W}$ denotes the masses of the $W$-bosons. This is the energy range for which our worldsheet theories are defined.

Note that even if ignore the classical argument and consider $4 \mathrm{~d}-2 \mathrm{~d}$ interaction terms, the form of the expression we obtained hints on a dramatic difference between strings for which condition (1.1) is satisfied and strings for which condition (1.1) is not satisfied. When (1.1) is satisfied, the sphere partition function suggests that it makes sense to separate the low-energy modes to modes that live on the string and modes that live in the bulk. When (1.1) is not satisfied, however, it seems unreasonable to separate the string and the bulk low-energy modes. Indeed, in the classical analysis of [1] we found that the assumption that we can separate to bulk and string low-energy modes leads to a contradiction when condition (1.1) is not satisfied, as one finds that the $2 \mathrm{~d}$ and $4 \mathrm{~d}$ modes are mixed already at the quadratic level and that the mixing terms become more and more important as we go down in energy.

The contribution corresponding to positive $K$ and $K^{\prime}$ that satisfy the condition (5.10) is expected to describe the low-energy dynamics around a $K$-string and a $K^{\prime}$-string wrapping the two-spheres $x_{0}^{2} / r^{2}+\left(x_{1}^{2}+x_{2}^{2}\right) / l^{2}=1$ and $x_{0}^{2} / r^{2}+\left(x_{3}^{2}+x_{4}^{2}\right) / \tilde{l}^{2}=1$ respectively, with interactions between the two worldsheet theories.

From now on, we will focus on the cases where the decoupling condition (5.10) is satisfied (if $N_{f}>N_{c}$ ). For simplicity, we will write the expression we find only for $K^{\prime}=0$, and will split it as $Z_{K, K^{\prime}=0,\left\{1,2, \ldots, N_{c}\right\}}^{B}=Z_{\text {overall }} \cdot Z_{S^{2}, K}$, where

$$
\begin{aligned}
Z_{\text {overall }}= & b^{\frac{\sum_{i} c_{i}}{C}\left(2 K^{2} b^{2}+2 N_{c} K\left(b^{2}+1\right)+2 i b K \sum_{a} \hat{\mu}_{a}\right)-2 i b K \sum_{i} \hat{\mu}_{i}-2 b \sum_{i, a} \Delta_{i a} K\left(i \mathbb{M}_{i a}+\frac{1}{2} \Delta_{i a} K b\right)} \\
& e^{-\frac{8 \pi^{2}}{e^{2}} \frac{N_{c}}{C^{2}}\left[2 i K b\left(-\sum_{a=1}^{N_{c}} \hat{\mu}_{a}+i N_{c} \frac{Q}{2}\right)-K^{2} b^{2}\right]} \\
& e^{\frac{8 \pi^{2}}{g^{2}}\left(\left(b^{2}+1\right) K+\sum_{a=1}^{N_{c}}\left(-2 i \frac{\hat{\mu}_{a} c a}{C} K b+2 i \frac{c_{a}^{2}}{C^{2}} K b \sum_{b=1}^{N_{c}} \hat{\mu}_{b}+\frac{c_{a}}{C}\left(\frac{c_{a}}{C} N_{c}-1\right)\left(b^{2}+1\right) K+\frac{c_{a}^{2}}{C^{2}} K^{2} b^{2}\right)\right)},
\end{aligned}
$$

\footnotetext{
${ }^{17}$ Note that this term is very similar to the $2 \mathrm{~d}$ superpotential (3.15). Indeed, the field $\tilde{\eta}$ can be thought of as a low-energy excitation of the field $\tilde{q}$ that is restricted to move in two-dimensions.
} 
and

$$
\begin{aligned}
Z_{S^{2}, K}=\sum_{\left\{k_{a}\right\} \in \Pi(K)} & {\left[\left(e^{\frac{8 \pi^{2}}{g^{2}}} b^{N_{f}-2 N_{c}}\right)^{\sum_{a=1}^{N_{c}} 2 i b \mathbb{M}_{a} k_{a}+\sum_{a=1}^{N_{c}} k_{a}^{2} b^{2}} \prod_{a, b=1}^{N_{c}} \prod_{r=0}^{k_{b}-1} \gamma\left(i b \mathbb{M}_{b a}-k_{a} b^{2}+r b^{2}\right)\right.} \\
& \prod_{a=1}^{N_{c}} \frac{\prod_{i \geq N_{c}+1, \Delta_{i a} K+k_{a} \geq 1} \prod_{r=0}^{\Delta_{i a} K+k_{a}-1} \gamma\left(i b \mathbb{M}_{i a}+b^{2} K \Delta_{i a}-(r+1) b^{2}\right)}{\prod_{i \geq N_{c}+1, \Delta_{i a} K+k_{a} \leq-1} \prod_{r=0}^{-\Delta_{i a} K-k_{a}-1} \gamma\left(i b \mathbb{M}_{i a}+b^{2} K \Delta_{i a}+r b^{2}\right)} \\
& \left.\times Z_{\text {inst }}^{\text {res }}\left(\mathbb{M}_{i j},\left\{k_{a}\right\}, q\right) Z_{\text {inst }}^{\text {res }}\left(\mathbb{M}_{i j},\left\{k_{a}\right\}, \bar{q}\right)\right]
\end{aligned}
$$

In the expression above we introduced the notation

$$
\begin{aligned}
\mathbb{M}_{i}\left(\left\{c_{k}\right\}, K\right) & =\hat{\mu}_{i}-\frac{c_{i}}{C}\left(\sum_{b=1}^{N_{c}} \hat{\mu}_{b}-i N_{c} \frac{Q}{2}-i K b\right), \\
\mathbb{M}_{i j} & =\mathbb{M}_{i}-\mathbb{M}_{j} .
\end{aligned}
$$

The expression for the instanton contributions to the residue, $Z_{\text {inst }}^{\text {res }}\left(\mathbb{M}_{i j},\left\{k_{a}\right\}, q\right)$, can be found in appendix A. In (5.11), sums over $a$ and $b$ indices are assumed to be from 1 to $N_{c}$ and sums over an $i$ index are assumed to be from $N_{c}+1$ to $N_{f}$, unless stated otherwise.

We thus conjecture that the low-energy fluctuations around the string solution are captured by a $2 \mathrm{~d} \mathcal{N}=(2,2)$ supersymmetric theory, whose sphere partition function is given, under some map of the parameters of the $4 \mathrm{~d}$ theory to the parameters of the $2 \mathrm{~d}$ theory, by equation (5.12). This conjecture is based on the assumption that under the $4 \mathrm{~d}-$ $2 \mathrm{~d}$ map of the parameters, equation (5.11) can be regarded as a regularization ambiguity of the two-sphere partition function (see the discussion around equation (4.29).)

\subsection{Identifications of worldsheet theories}

In this section, we will identify GLSMs whose two-sphere partition functions matches with equation (5.12), for all the cases where the condition (1.4) is satisfied. From the classical zero-modes point of view, this is the condition that the size-modes in equation (2.17) satisfy the F-term constraints trivially. This condition can also be derived from our localization analysis as the condition that the number of $\gamma$-functions that appear in (5.12) is the same for every choice of the partition $\left\{k_{a}\right\}$. The condition (1.4) allows us to identify worldsheet GLSMs that are weakly coupled when the $4 \mathrm{~d}$ theory is weakly coupled.

When the condition in equation (1.4) is not satisfied, the form of equation (5.12) changes dramatically. In some of these cases (see for example section 6.2.3) the expression (5.12) can be understood as a two-sphere partition function of a GLSM expanded around strong coupling.

\subsubsection{Strings with no $\tilde{q}$ excitations}

Let us consider equation (5.12) in the case where $c_{i} \geq c_{a}$ for all $1 \leq a \leq N_{c}$ and $N_{c}+1 \leq$ $i \leq N_{f}$. This condition arises from the zero-mode analysis as the condition that there are 
no size zero-modes involving excitations of $\tilde{q}$. See equation (2.17). It is not difficult to check that in this case

$$
Z_{S^{2}, K}\left(\mathbb{M}_{i}\left[c_{j}\right], c_{i}\right)=Z_{S^{2}, K}\left(\mathbb{M}_{i}\left[c_{j}\right], c_{i}=1\right) \prod_{a=1}^{N_{c}} \prod_{i=N_{c}+1}^{N_{f}} \prod_{r=0}^{\Delta_{i a} K-1} \gamma\left(i b \mathbb{M}_{i a}+b^{2} K \Delta_{i a}-(r+1) b^{2}\right) .
$$

Thus, we find that $Z_{S^{2}}$ is a product of two decoupled factors. The first factor in (5.14) is the two-sphere partition function of an $\mathcal{N}=(2,2)$ supersymmetric theory with gauge group $\mathrm{U}(K), N_{c}$ chiral multiplets in the fundamental representation, $N_{f}-N_{c}$ chiral multiplets in the anti-fundamental representation and one chiral multiplet in the adjoint representation. The parameters of this theory are given in terms of the parameters of the four-dimensional theory by

$$
\begin{aligned}
z & =b^{2 N_{c}-N_{f}} q & & \\
m_{a}-m_{b} & =\mu_{a}-\mu_{b}+\Delta_{b a} \sum_{c=1}^{N_{c}} \mu_{c}, & & R_{a}-R_{b}=\Delta_{a b} N_{c}\left(b^{2}+1\right)+2 \Delta_{a b} K b^{2}, \\
m_{a}+\tilde{m}_{j} & =\mu_{a}-\mu_{j}+\Delta_{j a} \sum_{c=1}^{N_{c}} \mu_{c}, & R_{a}+\tilde{R}_{j} & =\Delta_{a j} N_{c}\left(b^{2}+1\right)+2 \Delta_{a j} K b^{2}-2 b^{2}, \\
m_{X} & =0, & R_{X} & =-2 b^{2},
\end{aligned}
$$

where $m_{a}, R_{a}, a=1, \ldots, N_{c}$, are the twisted masses and $R$-charges of the fundamental chiral multiplets, $\tilde{m}_{j}, \tilde{R}_{j}, j=N_{c}+1, \ldots, N_{f}$, are the twisted masses and $R$-charges of the anti-fundamental chiral multiplets, and $m_{X}$ and $R_{X}$ are the twisted mass and $R$-charge of the adjoint chiral multiplet.

The second factor in (5.14) describes $K\left(\frac{N_{c}}{C} \sum_{i=N_{c}+1}^{N_{f}} c_{i}+N_{c}-N_{f}\right)$ free chiral multiplets, with twisted masses and $R$-charges

$$
\begin{aligned}
& m_{i, a, r}=\mu_{a}-\mu_{i}+\Delta_{i a} \sum_{b=1}^{N_{c}} \mu_{b}, \\
& R_{i, a, r}=\Delta_{a i} N_{c}\left(b^{2}+1\right)-2(r+1) b^{2},
\end{aligned}
$$

for $a=1, \ldots, N_{c}, i=N_{c}+1, \ldots, N_{f}, r=0, \ldots, \Delta_{i a} K-1$.

For a comparison between the low-energy spectrum of this theory and the classical spectrum of string zero-modes, see section 3.2.2.

\subsubsection{Strings with $q$ and $\tilde{q}$ excitations}

We now consider a more general case, in which

$$
\begin{aligned}
\Delta_{i a} \geq 0 & \text { for all } 1 \leq a \leq N_{c}, \quad N_{c}+1 \leq i \leq N_{q}, \\
-\Delta_{i a} \geq 1 & \text { for all } 1 \leq a \leq N_{c}, \quad N_{q}+1 \leq i \leq N_{f} .
\end{aligned}
$$

In terms of the classical size-modes given in equation (2.17), this is equivalent to requiring that there are no $\tilde{q}^{i}$ excitations for $N_{c}+1 \leq i \leq N_{q}$ and no $q_{i}$ excitations for $N_{q}+1 \leq i \leq N_{f}$. 
In this case, equation (5.12) reads

$$
\begin{aligned}
& Z_{S^{2}, K}=\sum_{\left\{k_{a}\right\} \in \Pi(K)}\left[\left(e^{\frac{8 \pi^{2}}{g^{2}}} b^{N_{f}-2 N_{c}}\right)^{\sum_{a=1}^{N_{c}} 2 i b \mathbb{M}_{a} k_{a}+\sum_{a=1}^{N_{c}} k_{a}^{2} b^{2}} \prod_{a, b=1}^{N_{c}} \prod_{r=0}^{k_{b}-1} \gamma\left(i b \mathbb{M}_{b a}-k_{a} b^{2}+r b^{2}\right)\right. \\
& \left.\prod_{a=1}^{N_{c}} \frac{\prod_{i=N_{c}+1}^{N_{q}} \prod_{r=0}^{\Delta_{i a} K+k_{a}-1} \gamma\left(i b \mathbb{M}_{i a}+b^{2} K \Delta_{i a}-(r+1) b^{2}\right)}{\prod_{i=N_{q}+1}^{N_{f}} \prod_{r=0}^{-\Delta_{i a} K-k_{a}-1} \gamma\left(i b \mathbb{M}_{i a}+b^{2} K \Delta_{i a}+r b^{2}\right)} Z_{\text {inst }}^{\text {res }}\left(\mathbb{M}_{i j},\left\{k_{a}\right\}, q\right) Z_{\text {inst }}^{\text {res }}\left(\mathbb{M}_{i j},\left\{k_{a}\right\}, \bar{q}\right)\right] .
\end{aligned}
$$

Our ansatz is that the worldsheet theory, in this case, is the low-energy limit of an $\mathcal{N}=(2,2)$ GLSM with a $\mathrm{U}(K)$ gauge group and the following matter content:

- 1 adjoint chiral multiplet, $X$, with complexified twisted mass $M_{X}$,

- $N_{c}$ fundamental chiral multiplets, $\psi_{a}, a=1, \ldots, N_{c}$, with complexified twisted masses $M_{a}$,

- $N_{f}-N_{c}$ anti-fundamental chiral multiplets, $\tilde{\psi}_{j}, j=N_{c}+1, \ldots, N_{f}$, with complexified twisted masses $\tilde{M}_{j}$,

- neutral decoupled chiral multiplets, $\eta_{i, a, r}$, for $a=1, \ldots, N_{c}, i=N_{c}+1, \ldots, N_{q}$, $r=0, \ldots, \Delta_{i a} K-1$, with complexified twisted masses $M_{i, a, r}$,

- neutral chiral multiplets $\tilde{\eta}_{i, a, r}$, for $a=1, \ldots, N_{c}, i=N_{q}+1, \ldots, N_{f}, r=0, \ldots, \Delta_{a i} K-1$, with complexified twisted masses $\tilde{M}_{i, a, r}=i-M_{a}-\tilde{M}_{i}-\left(K \Delta_{a i}-r-1\right) M_{X}$.

The relation between the complexified twisted masses allows a superpotential of the form

$$
W=\sum_{a=1}^{N_{c}} \sum_{i=N_{q}+1}^{N_{f}} \sum_{r=0}^{\Delta_{a i} K-1} \alpha_{r} \tilde{\eta}_{i, a, r} \tilde{\psi}_{i} X^{\Delta_{a i} K-r-1} \psi_{a}
$$

The two-sphere partition function does not depend on the superpotential couplings $\alpha_{r}$, and therefore can be written as $Z_{\text {neutral }} \cdot Z_{\text {charged }}$, where

$$
Z_{\text {neutral }}=\prod_{i=N_{c}+1}^{N_{q}} \prod_{a=1}^{N_{c}} \prod_{r=0}^{\Delta_{i a} K-1} \gamma\left(-i M_{i, a, r}\right) \prod_{i=N_{q}+1}^{N_{f}} \prod_{a=1}^{N_{c}} \prod_{r=0}^{\Delta_{a i} K-1} \gamma\left(-i \tilde{M}_{i, a, r}\right)
$$

describes the neutral fields $\eta$ and $\tilde{\eta}$, and $Z_{\text {charged }}$ is given by equation (4.23), which we repeat here for the convenience of the reader,

$$
\begin{aligned}
& Z_{\text {charged }}= \\
& \sum_{\left\{k_{a}\right\} \in \Pi(K)}\left[(z \bar{z})^{-i\left(\sum_{a=1}^{N_{c}} k_{a} M_{a}+\frac{M_{X}}{2}\left(\sum_{a=1}^{N_{c}} k_{a}^{2}-K\right)\right)} \frac{\prod_{a, b=1}^{N_{c}} \prod_{r=0}^{k_{a}-1} \gamma\left(i\left(M_{a}-M_{b}\right)+i\left(r-k_{b}\right) M_{X}\right)}{\prod_{j=N_{c}+1}^{N_{f}} \prod_{a=1}^{N_{c}} \prod_{r=0}^{k_{a}-1} \gamma\left(1+i M_{a}+i \tilde{M}_{j}+i r M_{X}\right)}\right. \\
& \left.\quad Z_{\left\{k_{a}\right\}}^{\mathrm{vort}}(z) Z_{\left\{k_{a}\right\}}^{\mathrm{vort}}(\bar{z})\right],
\end{aligned}
$$

where $Z_{\left\{k_{a}\right\}}^{\text {vort }}(z)$ is given by equation (A.7). 
Due to the relation between the masses, some of the terms cancel when we multiply $Z_{\text {neutral }}$ with $Z_{\text {charged }}$ and we are left with

$$
\begin{aligned}
& \prod_{i=N_{c}+1}^{Z_{\text {neutral }} \cdot Z_{\text {charged }}=} \prod_{r=1}^{N_{q}} \prod_{r=0}^{N_{c}} \gamma\left(-i M_{i, a, r}\right) \prod_{i=N_{q}+1}^{\Delta_{i a} K-1} \prod_{a=1}^{N_{c}} \prod_{r=0}^{N_{c}} \gamma\left(-i \tilde{M}_{i, a, r}\right) \\
& \quad \sum_{\left\{k_{a}\right\} \in \Pi(K)}^{\Delta_{a i} K-K-1}(z \bar{z})^{-i\left(\sum_{a=1}^{N_{c}} k_{a} M_{a}+\frac{M_{X}}{2}\left(\sum_{a=1}^{N_{c}} k_{a}^{2}-K\right)\right)} \frac{\prod_{a, b=1}^{N_{c}} \prod_{r=0}^{k_{a}-1} \gamma\left(i\left(M_{a}-M_{b}\right)+i\left(r-k_{b}\right) M_{X}\right)}{\prod_{j=N_{c}+1}^{N_{q}} \prod_{a=1}^{N_{c}} \prod_{r=0}^{k_{a}-1} \gamma\left(1+i M_{a}+i \tilde{M}_{j}+i r M_{X}\right)} \\
& \prod_{a=1}^{N_{c}} \prod_{j=N_{q}+1}^{N_{f}} \prod_{r=1}^{K-k_{a}} \gamma\left(1+i M_{a}+i \tilde{M}_{j}+i(K-r) M_{X}\right) Z_{\left\{k_{a}\right\}}^{\text {vort }}(z) Z_{\left\{k_{a}\right\}}^{\text {vort }}(\bar{z}) .
\end{aligned}
$$

To compare equation (5.18) with equation (5.22) it is convenient to rewrite equation (5.18) as

$$
\begin{aligned}
Z_{S^{2}, K}= & \sum_{\left\{k_{a}\right\} \in \Pi(K)}\left[\left(e^{\frac{8 \pi^{2}}{g^{2}}} b^{N_{f}-2 N_{c}}\right)^{\sum_{a=1}^{N_{c}} 2 i b \mathbb{M}_{a} k_{a}+\sum_{a=1}^{N_{c}} k_{a}^{2} b^{2}} \prod_{a, b=1}^{N_{c}} \prod_{r=0}^{k_{b}-1} \gamma\left(i b \mathbb{M}_{b a}-k_{a} b^{2}+r b^{2}\right)\right. \\
& \prod_{a=1}^{N_{c}} \prod_{i=N_{c}+1}^{N_{q}} \prod_{r=0}^{k_{a}-1} \gamma\left(i b \mathbb{M}_{i a}-(r+1) b^{2}\right) \prod_{a=1}^{N_{c}} \prod_{i=N_{q}+1}^{N_{f}} \prod_{r=1}^{K-k_{a}} \gamma\left(1+i b \mathbb{M}_{a i}+(K-r+1) b^{2}\right) \\
& \left.Z_{\text {inst }}^{\text {res }}\left(\mathbb{M}_{i j},\left\{k_{a}\right\}, q\right) Z_{\text {inst }}^{\text {res }}\left(\mathbb{M}_{i j},\left\{k_{a}\right\}, \bar{q}\right)\right] \times \\
& \prod_{a=1}^{N_{c}} \prod_{i=N_{c}+1}^{N_{q}} \prod_{r=0}^{\Delta_{i a} K-1} \gamma\left(i b \mathbb{M}_{i a}+b^{2} K \Delta_{i a}-(r+1) b^{2}\right) \\
& \times \prod_{a=1}^{N_{c}} \prod_{i=N_{q}+1}^{N_{f}} \prod_{r=0}^{-K-\Delta_{i a} K-1} \gamma\left(1+i b \mathbb{M}_{a i}-\left(r+K \Delta_{i a}\right) b^{2}\right)
\end{aligned}
$$

Equation (5.23) maps to equation $(5.22)^{18}$ under the dictionary

$$
\begin{aligned}
z & =b^{2 N_{c}-N_{f}} q \\
M_{a}-M_{b} & =b \mathbb{M}_{a b}, \\
M_{a}+\tilde{M}_{i} & =b \mathbb{M}_{a i}-i b^{2}, \\
M_{X} & =-i b^{2}, \\
M_{i, a, r} & =b \mathbb{M}_{a i}+i\left(K \Delta_{i a}-r-1\right) b^{2}, \\
\tilde{M}_{i, a, r} & =i+b \mathbb{M}_{i a}+i\left(K \Delta_{a i}-r\right) b^{2} .
\end{aligned}
$$

\footnotetext{
${ }^{18} \mathrm{Up}$ to an overall factor, which we interpret as a supersymmetric counterterm.
} 
The $2 d$ masses and $R$-charges are therefore given by

$$
\begin{aligned}
m_{a}-m_{b} & =\mu_{a}-\mu_{b}+\Delta_{b a} \sum_{c=1}^{N_{c}} \mu_{c}, & R_{a}-R_{b} & =\Delta_{a b} N_{c}\left(b^{2}+1\right)+2 \Delta_{a b} K b^{2}, \\
m_{a}+\tilde{m}_{j} & =\mu_{a}-\mu_{j}+\Delta_{j a} \sum_{c=1}^{N_{c}} \mu_{c}, & R_{a}+\tilde{R}_{j} & =\Delta_{a j} N_{c}\left(b^{2}+1\right)+2 \Delta_{a j} K b^{2}-2 b^{2}, \\
m_{X} & =0, & R_{X} & =-2 b^{2}, \\
m_{i, a, r} & =\mu_{a}-\mu_{i}+\Delta_{i a} \sum_{b=1}^{N_{c}} \mu_{b}, & R_{i, a, r} & =N_{c} \Delta_{a i}\left(b^{2}+1\right)-2(r+1) b^{2}, \\
\tilde{m}_{i, a, r} & =\mu_{i}-\mu_{a}+\Delta_{a i} \sum_{b=1}^{N_{c}} \mu_{b}, & \tilde{R}_{i, a, r} & =\left(N_{c} \Delta_{i a}+2\right)\left(b^{2}+1\right)-2(r+1) b^{2} .
\end{aligned}
$$

For a comparison between the low-energy spectrum of this theory and the classical spectrum of string zero-modes, see section 3.3.2.

\section{Strings in $N_{c}=2, N_{f}=4$ SQCD under triality}

In this section we will discuss the string configurations in the $N_{c}=2, N_{f}=4$ case, with $\mathrm{U}(1)$ charge assignments that are related by triality to the well-studied equal charge case. The triality provides predictions for the worldsheet theories, which we test against our localization results. The triality also provides examples in which the weak coupling regime of the $4 \mathrm{~d}$ theory is mapped to the strong coupling regime of the worldsheet theory. We will discuss such an example in detail.

\subsection{SO(8) symmetry and triality}

Let us start by reviewing the triality of $\mathcal{N}=2 \mathrm{SU}(2)$ gauge theory with four fundamental hypermultiplets. The matter content of this theory can be organized in eight $\mathcal{N}=1$ chiral multiplets, $Q_{i}^{a}, \tilde{Q}_{a}^{i}$, where $i=1, \ldots, 4$ is the flavor index and $a=1,2$ is the color index. $Q$ and $\tilde{Q}$ transform, respectively, in the fundamental and anti-fundamental representations of $\mathrm{SU}(2)$. If the hypermultiplets are massless, the theory has a classical $\mathrm{SO}(8)$ flavor symmetry under which

$$
Q_{v}^{a} \equiv\left(Q_{1}^{a}+\epsilon^{a b} \tilde{Q}_{b}^{1}, i Q_{1}^{a}-i \epsilon^{a b} \tilde{Q}_{b}^{1}, \ldots, Q_{4}^{a}+\epsilon^{a b} \tilde{Q}_{b}^{4}, i Q_{4}^{a}-i \epsilon^{a b} \tilde{Q}_{b}^{4}\right)^{T}
$$

transforms in the vector representation, which will be denoted by $8_{\mathrm{v}}$. In the quantum level, the $\mathrm{SO}(8)$ symmetry is enhanced to $\operatorname{Spin}(8)$.

Hypermultiplet masses can be introduced by weakly gauging four U(1) flavor symmetries and introducing vacuum expectation values for the four gauge multiplets scalars 
$\left\langle a_{i}\right\rangle=\mu_{i}$. In the $8_{\mathrm{v}}$ basis, the mass matrix can be written as ${ }^{19}$

$$
\left(\begin{array}{llll}
\varepsilon \mu_{1} & & & \\
& \varepsilon \mu_{2} & & \\
& & \varepsilon \mu_{3} & \\
& & & \varepsilon \mu_{4}
\end{array}\right), \quad \varepsilon=\left(\begin{array}{cc} 
& 1 \\
-1 &
\end{array}\right) .
$$

If the masses are all distinct, this breaks the $\mathrm{SO}(8)$ flavor symmetry to its maximal torus $\mathrm{SO}(2) \times \mathrm{SO}(2) \times \mathrm{SO}(2) \times \mathrm{SO}(2)$.

The group $\mathrm{SO}(8)$ has an $\mathbf{S}_{3}$ outer automorphism group, which exchanges the three 8dimensional irreducible representations $8_{\mathrm{v}}, 8_{\mathrm{s}}$ and $8_{\mathrm{c}}$, where $8_{\mathrm{s}}$ and $8_{\mathrm{c}}$ are the two spinorial representations. The six elements in $\mathbf{S}_{3}$ can be generated using two transformations, $S$ and $T$, via $\{1, S, T, S T, T S, S T S\}$. The action of $T$ on the Cartan generators is given by

$$
\begin{aligned}
& M_{12} \rightarrow M_{12} \\
& M_{34} \rightarrow M_{34} \\
& M_{56} \rightarrow M_{56} \\
& M_{78} \rightarrow-M_{78} .
\end{aligned}
$$

and the action of $S$ is given by

$$
\begin{aligned}
& M_{12} \rightarrow \frac{1}{2}\left(M_{12}+M_{34}+M_{56}+M_{78}\right) \\
& M_{34} \rightarrow \frac{1}{2}\left(M_{12}+M_{34}-M_{56}-M_{78}\right) \\
& M_{56} \rightarrow \frac{1}{2}\left(M_{12}-M_{34}+M_{56}-M_{78}\right) \\
& M_{78} \rightarrow \frac{1}{2}\left(M_{12}-M_{34}-M_{56}+M_{78}\right),
\end{aligned}
$$

where $M_{I J}$ correspond to the $\mathrm{SO}(8)$ generators $\left(M_{I J}\right)_{K L}=-i\left(\delta_{I K} \delta_{J L}-\delta_{I L} \delta_{J K}\right)$.

The $\mathbf{S}_{3}$ outer-automorphism of the $\mathrm{SO}(8)$ flavor symmetry of the massless theory turns out to be the duality group of the massive theory, where the masses are assigned with the transformation properties that follow from (6.4)-(6.5) and (6.3). The $\mathrm{SU}(2)$ coupling $q \equiv e^{2 \pi i \tau}$ transforms as $q \rightarrow \frac{q}{q-1}$ under $T$ and $q \rightarrow 1-q$ under $S$. Thus, under $T$

$$
\begin{aligned}
q & \rightarrow \frac{q}{q-1}, \\
\mu_{1} & \rightarrow \mu_{1}, \\
\mu_{2} & \rightarrow \mu_{2}, \\
\mu_{3} & \rightarrow \mu_{3}, \\
\mu_{4} & \rightarrow-\mu_{4},
\end{aligned}
$$

\footnotetext{
The map between the two conventions is

$$
\begin{array}{ll}
\mu_{1}=m_{a}+m_{d}, & \mu_{3}=m_{c}+m_{b}, \\
\mu_{2}=-m_{a}+m_{d}, & \mu_{4}=-m_{c}+m_{b} .
\end{array}
$$
}

${ }^{19}$ There is another basis that is very common in the literature, in which four mass parameters, $m_{a}, m_{b}, m_{c}, m_{d}$ are obtained by weakly gauging the $\mathrm{SU}(2)_{a} \times \mathrm{SU}(2)_{b} \times \mathrm{SU}(2)_{c} \times \mathrm{SU}(2)_{d}$ subgroup of $\mathrm{SO}(8)$.

In this work we will always use mass basis (6.3). 
and under $S$,

$$
\begin{aligned}
q & \rightarrow 1-q, \\
\mu_{1} & \rightarrow \frac{1}{2}\left(\mu_{1}+\mu_{2}+\mu_{3}+\mu_{4}\right), \\
\mu_{2} & \rightarrow \frac{1}{2}\left(\mu_{1}+\mu_{2}-\mu_{3}-\mu_{4}\right), \\
\mu_{3} & \rightarrow \frac{1}{2}\left(\mu_{1}-\mu_{2}+\mu_{3}-\mu_{4}\right), \\
\mu_{4} & \rightarrow \frac{1}{2}\left(\mu_{1}-\mu_{2}-\mu_{3}+\mu_{4}\right) .
\end{aligned}
$$

The triality of SU(2) SQCD was first discovered by Seiberg and Witten [30], who observed that the Seiberg-Witten curve is invariant under the transformations (6.6) and (6.7). The geometric origin of this triality was understood by Gaiotto. In [38], Gaiotto constructed a family of $\mathrm{SU}(2)$ superconformal $\mathcal{N}=2$ theories by compactifying the $\mathcal{N}=(2,0)$ six-dimensional theory of type $A_{1}$ on punctured Riemann surfaces. The four-dimensional theories are then determined by the choice of the Riemann surface and the number of punctures. The duality group of the four-dimensional theory is understood in Gaiotto's construction as the symmetry group on the set of trinion decompositions of the punctured Riemann surface - the set of different ways to obtain this surface by sewing together three-punctured spheres. In the case of $\mathrm{SU}(2)$ with four fundamental hypermultiplets, the Riemann surface is the four-punctured sphere, which can be sewn from two three-punctured spheres in three ways. The group of permutations of these three sewings is the $\mathbf{S}_{3}$ triality group.

The triality can also be interpreted, using the AGT conjecture [39], as the crossing symmetry of four-point functions in Liouville theory on the four punctured sphere. In [39] Alday, Gaiotto and Tachikawa argued that the partition function of $\mathcal{N}=2 N_{f}=4 \mathrm{SU}(2)$ theory in the $\Omega$-background is equal to a conformal block in Liouville theory. Due to the relation between the $\Omega$-background partition function and the four-ellipsoid partition function, this implies that the four-ellipsoid partition function is equal to the four-point function in Liouville theory.

To discuss string configurations, we need to gauge a $\mathrm{U}(1)$ flavor symmetry. In the equal charge case, the $\mathrm{U}(1)$ generator is embedded in $\mathrm{SO}(8)$ as

$$
T_{B}=\left(\begin{array}{llll}
\varepsilon & & \\
& \varepsilon & & \\
& & \varepsilon & \\
& & \varepsilon
\end{array}\right) .
$$

Using (6.4)-(6.5) we see that under $T$, the $\mathrm{SO}(8)$ generator (6.8) transforms into

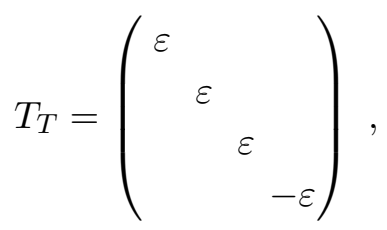


and that under $S,(6.8)$ transforms into

$$
T_{S}=\left(\begin{array}{cccc}
2 \varepsilon & & \\
& 0 & & \\
& & 0 & \\
& & & 0
\end{array}\right) .
$$

As the choice of $\mathrm{U}(1)$ breaks $\mathbf{S}_{3}$, the strings are configurations in theories that are not self-dual under triality. However, in the limit where the $\mathrm{U}(1)$ gauge coupling $e$ is infinitesimally small, we expect the strings to be related by triality to strings in different theories in which the dual U(1)'s are gauged. In particular, if the $\mathrm{U}(1)_{T}$ or $\mathrm{U}(1)_{S}$ flavor symmetry is gauged, the string worldsheet theory should be the one that was obtained in the equal charge gauging (for $N_{c}=2, N_{f}=4$ ) and the map between the $4 \mathrm{~d}$ and $2 \mathrm{~d}$ parameters should be the composition of the map (4.24) with the transformations (6.6) or (6.7), respectively. Below, we check this claim by studying the two-sphere partition functions that result from our analysis for the minimal $(K=1)$ string configurations in the three cases where (6.8), (6.9) or (6.10) are gauged.

To avoid confusion, let us end this section with a comment about the definition of the complexified gauge coupling. It is common in the literature to distinguish between the coupling that is defined using the classical prepotential, $\tau_{\mathrm{UV}}$, and the one defined using the quantum corrected prepotential, $\tau_{\mathrm{IR}}$. The early works on Seiberg-Witten theories use $\tau_{\mathrm{IR}}$, while in recent works that use localization techniques, $\tau_{\mathrm{UV}}$ appears naturally. Another source of potential confusion is that the definition of the microscopic prepotential is renormalization scheme dependent. As a result, the relation between $\tau_{\mathrm{UV}}$ and $\tau_{\mathrm{IR}}$ is scheme dependent. When the moduli space is parametrized in terms of $\tau_{\mathrm{IR}}$, the duality group is $\operatorname{SL}(2, \mathbb{Z})$, acting on the coupling $\tau_{\mathrm{IR}} \operatorname{as}^{20}$

$$
2 \tau_{\mathrm{IR}} \rightarrow \frac{2 a \tau_{\mathrm{IR}}+b}{2 c \tau_{\mathrm{IR}}+d}, \quad a, b, c, d \in \mathbb{Z}, \quad a d-b c=1
$$

In the regularization scheme used by Nekrasov [34], the prepotential (in the massless limit) determines the following relation between $\tau_{\mathrm{UV}}$ and $\tau_{\mathrm{IR}}$

$$
q \equiv e^{2 \pi i \tau_{\mathrm{UV}}}=\lambda\left(2 \tau_{\mathrm{IR}}\right)
$$

where $\lambda$ is the modular lambda-function, which is defined on the upper half plane. $\lambda\left(2 \tau_{\mathrm{IR}}\right)$ is invariant under the subgroup $\Gamma(2) \subset \mathrm{SL}(2, \mathbb{Z})$, which is generated by $\tau_{\mathrm{IR}} \rightarrow \tau_{\mathrm{IR}}+1$ and $\tau_{\mathrm{IR}} \rightarrow \frac{\tau_{\mathrm{IR}}}{1-4 \tau_{\mathrm{IR}}}$. Under $2 \tau_{\mathrm{IR}} \rightarrow 2 \tau_{\mathrm{IR}}+1$ and $2 \tau_{\mathrm{IR}} \rightarrow-\frac{1}{2 \tau_{\mathrm{IR}}}, \lambda\left(2 \tau_{\mathrm{IR}}\right)$ transforms as $\lambda \rightarrow \frac{\lambda}{\lambda-1}$ and $\lambda \rightarrow 1-\lambda$ respectively. Therefore, in terms of the coupling $q$, the duality group is $\mathrm{SL}(2, \mathbb{Z}) / \Gamma(2)=\mathbf{S}_{3}$ with the $T$ and $S$ transformations acting on $q$ as described above.

\footnotetext{
${ }^{20}$ The unconventional $\mathrm{SL}(2, \mathbb{Z})$ transformation is due to the normalization of $\tau_{\mathrm{IR}}$, which is usually normalized as $\tau_{\mathrm{IR}}^{\prime}=2 \tau_{\mathrm{IR}}=\frac{\theta}{\pi}+\frac{8 \pi i}{g^{2}}$.
} 


\subsection{Strings in $N_{c}=2, N_{f}=4$ SQCD under triality - localization analysis}

The four-ellipsoid partition function for an $\mathrm{SU}(2) \times \mathrm{U}(1)$ gauge theory with four hypermultiplets in the fundamental representation of $\mathrm{SU}(2)$ can be written as

$$
\begin{aligned}
Z_{S_{b}^{4}}= & \int d \hat{a} d \hat{a}^{\prime} e^{-\frac{16 \pi^{2}}{g^{2}} \hat{a}^{2}} e^{-\frac{16 \pi^{2}}{e^{2}} \hat{a}^{\prime 2}} e^{32 i \pi^{2} \hat{\xi} \hat{a}^{\prime}} \Upsilon_{b}(2 i \hat{a}) \Upsilon_{b}(-2 i \hat{a}) \\
& \left(\prod_{j=1}^{4} \Upsilon_{b}\left(i \hat{a}-i\left(c_{j} \hat{a}^{\prime}+\hat{\mu}_{j}\right)+\frac{Q}{2}\right) \Upsilon_{b}\left(-i \hat{a}-i\left(c_{j} \hat{a}^{\prime}+\hat{\mu}_{j}\right)+\frac{Q}{2}\right)\right)^{-1}\left|Z_{\text {inst }}\left(\hat{a}, \hat{a}^{\prime}, c_{j}, \hat{\mu}_{j}\right)\right|^{2},
\end{aligned}
$$

where $a$ is the Coulomb branch parameter for the Cartan of $\mathrm{SU}(2)$ and $a^{\prime}$ is the Coulomb branch parameter for the $\mathrm{U}(1)$ factor. As before, we have denoted by $c_{j}$ the $\mathrm{U}(1)$ charge of the $j$ 'th hypermultiplet, and by $\mu_{j}$ its mass. For the equal-charge $\mathrm{U}(1)_{B}(6.8)$ and its $T$ - and $S$-dual U(1)'s, (6.9) and (6.10),

$$
\begin{array}{ll}
\mathrm{U}(1)_{B} ; & c_{1}=c_{2}=c_{3}=c_{4}=1, \\
\mathrm{U}(1)_{T} ; & c_{1}=c_{2}=c_{3}=1, \quad c_{4}=-1, \\
\mathrm{U}(1)_{S} ; & c_{1}=2, \quad c_{2}=c_{3}=c_{4}=0 .
\end{array}
$$

In this section we will apply our prescription to the minimal $(K=1)$ strings in these three dual cases, and will compare the duality predictions for the worldsheet theories with the output of our analysis.

\subsubsection{The $c_{i}=1$ case}

This case is a special case of section 4.2. In the $N_{f}=2 N_{c}=4$ case, equation (4.21) reads

$$
\begin{aligned}
Z_{K=1,\{1,2\}}= & e^{\frac{4 \pi^{2}}{e^{2}}\left(3 b^{2}+2+2 i b\left(\hat{\mu}_{1}+\hat{\mu}_{2}\right)\right)} b^{4+6 b^{2}+2 i b\left(\hat{\mu}_{1}+\hat{\mu}_{2}-\hat{\mu}_{3}-\hat{\mu}_{4}\right)} \gamma\left(-b^{2}\right) \\
& {\left[(q \bar{q})^{i b\left(\hat{\mu}_{2}-\hat{\mu}_{1}\right) / 2-b^{2} / 4} \frac{\gamma\left(i b\left(\hat{\mu}_{1}-\hat{\mu}_{2}\right)\right)}{\prod_{j=3,4} \gamma\left(1+i b\left(\hat{\mu}_{1}-\hat{\mu}_{j}\right)+b^{2}\right)}\right.} \\
& { }_{2} F_{1}\left(-i b\left(\hat{\mu}_{1}-\hat{\mu}_{3}\right)-b^{2},-i b\left(\hat{\mu}_{1}-\hat{\mu}_{4}\right)-b^{2}, 1-i b\left(\hat{\mu}_{1}-\hat{\mu}_{2}\right) \mid q\right) \\
& \left.{ }_{2} F_{1}\left(-i b\left(\hat{\mu}_{1}-\hat{\mu}_{3}\right)-b^{2},-i b\left(\hat{\mu}_{1}-\hat{\mu}_{4}\right)-b^{2}, 1-i b\left(\hat{\mu}_{1}-\hat{\mu}_{2}\right) \mid \bar{q}\right)+\mu_{1} \leftrightarrow \mu_{2}\right],
\end{aligned}
$$

where we have written the result for $\left\{l_{1}, l_{2}\right\}=\{1,2\}$ for simplicity. The dictionary (4.24) is:

$$
\begin{aligned}
z & =q, \\
M_{1}-M_{2} & =b\left(\hat{\mu}_{1}-\hat{\mu}_{2}\right), \\
M_{1}+\tilde{M}_{1} & =b\left(\hat{\mu}_{1}-\hat{\mu}_{3}\right)-i b^{2}, \\
M_{1}+\tilde{M}_{2} & =b\left(\hat{\mu}_{1}-\hat{\mu}_{4}\right)-i b^{2}, \\
M_{X} & =-i b^{2},
\end{aligned}
$$

where $M_{1,2}, \tilde{M}_{1,2}$, and $M_{X}$ are dimensionless complexified twisted masses for the positively charged, negatively charged and adjoint chiral multiplets respectively. 
The dictionary (6.18) maps (6.17) to the two-sphere partition function of the desired GLSM, ${ }^{21}$

$$
\begin{aligned}
Z_{S^{2}}= & 2 \pi(z \bar{z})^{-i M_{1}} \gamma\left(-i M_{X}\right) \frac{\gamma\left(-i \tilde{M}_{1}-i M_{1}\right) \gamma\left(-i \tilde{M}_{2}-i M_{1}\right)}{\gamma\left(1+i M_{2}-i M_{1}\right)} \\
& \times_{2} F_{1}\left(-i M_{1}-i \tilde{M}_{1},-i M_{1}-i \tilde{M}_{2}, 1+i M_{2}-i M_{1} \mid z\right) \\
& \times_{2} F_{1}\left(-i M_{1}-i \tilde{M}_{1},-i M_{1}-i \tilde{M}_{2}, 1+i M_{2}-i M_{1} \mid \bar{z}\right) \\
& +M_{1} \leftrightarrow M_{2} .
\end{aligned}
$$

\subsubsection{T-dual of the $c_{i}=1$ case}

Due to the fact that the fundamental representation of $\mathrm{SU}(2)$ is pseudo-real, the transformation $c_{4} \rightarrow-c_{4}$ is a classical symmetry of the massless theory. However, this symmetry is broken by non-perturbative contributions [30]. Therefore, the difference between the theory in which (6.14) is gauged and the theory in which (6.15) is gauged, is manifested only through instanton contributions, in addition to a $\mu_{4} \rightarrow-\mu_{4}$ transformation in the perturbative contributions. Using the instanton analysis of appendix A, we find in this case

$$
\begin{aligned}
Z_{K=1,\{1,2\}}= & e^{\frac{4 \pi^{2}}{e^{2}}\left(3 b^{2}+2+2 i b\left(\hat{\mu}_{1}+\hat{\mu}_{2}\right)\right)} b^{4+6 b^{2}+2 i b\left(\hat{\mu}_{1}+\hat{\mu}_{2}-\hat{\mu}_{3}+\hat{\mu}_{4}\right)} \gamma\left(-b^{2}\right) \\
& {\left[(q \bar{q})^{i b\left(\hat{\mu}_{2}-\hat{\mu}_{1}\right) / 2-b^{2} / 4} \frac{\gamma\left(i b\left(\hat{\mu}_{1}-\hat{\mu}_{2}\right)\right)}{\gamma\left(1+i b\left(\hat{\mu}_{1}-\hat{\mu}_{3}\right)+b^{2}\right) \gamma\left(1+i b\left(\hat{\mu}_{1}+\hat{\mu}_{4}\right)+b^{2}\right)}\right.} \\
& { }_{2} F_{1}\left(-i b\left(\hat{\mu}_{1}-\hat{\mu}_{3}\right)-b^{2}, i b\left(\hat{\mu}_{2}+\hat{\mu}_{4}\right)+b^{2}+1,1-i b\left(\hat{\mu}_{1}-\hat{\mu}_{2}\right) \mid q\right) \\
& \left.{ }_{2} F_{1}\left(-i b\left(\hat{\mu}_{1}-\hat{\mu}_{3}\right)-b^{2}, i b\left(\hat{\mu}_{2}+\hat{\mu}_{4}\right)+b^{2}+1,1-i b\left(\hat{\mu}_{1}-\hat{\mu}_{2}\right) \mid \bar{q}\right)+\mu_{1} \leftrightarrow \mu_{2}\right] .
\end{aligned}
$$

Using the hypergeometric function identity (B.2) to rewrite this expression, we get

$$
\begin{aligned}
& Z_{K=1,\{1,2\}}= \\
& e^{\frac{4 \pi^{2}}{e^{2}}\left(3 b^{2}+2+2 i b\left(\hat{\mu}_{1}+\hat{\mu}_{2}\right)\right)} b^{4+6 b^{2}+2 i b\left(\hat{\mu}_{1}+\hat{\mu}_{2}-\hat{\mu}_{3}+\hat{\mu}_{4}\right)} \gamma\left(-b^{2}\right)(q \bar{q})^{-b^{2} / 4}[(1-q)(1-\bar{q})]^{b^{2}} \\
& \quad\left[(q \bar{q})^{i b\left(\hat{\mu}_{2}-\hat{\mu}_{1}\right) / 2}[(1-q)(1-\bar{q})]^{i b\left(\hat{\mu}_{1}-\hat{\mu}_{3}\right)} \frac{\gamma\left(i b\left(\hat{\mu}_{1}-\hat{\mu}_{2}\right)\right)}{\gamma\left(1+i b\left(\hat{\mu}_{1}-\hat{\mu}_{3}\right)+b^{2}\right) \gamma\left(1+i b\left(\hat{\mu}_{1}+\hat{\mu}_{4}\right)+b^{2}\right)}\right. \\
& { }_{2} F_{1}\left(-i b\left(\hat{\mu}_{1}-\hat{\mu}_{3}\right)-b^{2},-i b\left(\hat{\mu}_{1}+\hat{\mu}_{4}\right)-b^{2}, 1-i b\left(\hat{\mu}_{1}-\hat{\mu}_{2}\right) \mid \frac{q}{q-1}\right) \\
& \left.{ }_{2} F_{1}\left(-i b\left(\hat{\mu}_{1}-\hat{\mu}_{3}\right)-b^{2},-i b\left(\hat{\mu}_{1}+\hat{\mu}_{4}\right)-b^{2}, 1-i b\left(\hat{\mu}_{1}-\hat{\mu}_{2}\right) \mid \frac{\bar{q}}{\bar{q}-1}\right)+\mu_{1} \leftrightarrow \mu_{2}\right] .
\end{aligned}
$$

The composition of the dictionary (6.18) and the $T$-transformation (6.6) gives the

\footnotetext{
${ }^{21} \mathrm{Up}$ to the overall factor (4.29), which is interpreted as a regularization ambiguity of $Z_{S^{2}}$.
} 
dictionary

$$
\begin{aligned}
z & =\frac{q}{q-1}, \\
M_{1}-M_{2} & =b\left(\hat{\mu}_{1}-\hat{\mu}_{2}\right), \\
M_{1}+\tilde{M}_{1} & =b\left(\hat{\mu}_{1}-\hat{\mu}_{3}\right)-i b^{2}, \\
M_{1}+\tilde{M}_{2} & =b\left(\hat{\mu}_{1}+\hat{\mu}_{4}\right)-i b^{2}, \\
M_{X} & =-i b^{2} .
\end{aligned}
$$

As expected, this dictionary maps the expression in (6.21) to the two-sphere partition function (6.19), up to the multiplicative factor

$$
\begin{aligned}
& e^{\frac{4 \pi^{2}}{e^{2}}\left(3 b^{2}+2+2 i b\left(\hat{\mu}_{1}+\hat{\mu}_{2}\right)\right)}\left(b^{2}\right)^{2+3 b^{2}+i l\left(m_{1}+m_{2}+\tilde{m}_{1}+\tilde{m}_{2}\right)} \\
& \quad \times(z \bar{z})^{\frac{i\left(M_{1}+M_{2}\right)}{2}-\frac{b^{2}}{4}}[(1-z)(1-\bar{z})]^{-i l \frac{m_{1}+m_{2}}{2}-i l \tilde{m}_{1}-\frac{3 b^{2}}{4}} .
\end{aligned}
$$

Note that the charge assignment (6.15) obeys the condition (1.4). The strings discussed in this section are therefore a special case of the strings discussed in section 5.2.2, for which we identified the worldsheet sphere partition functions as sphere partition functions of GLSMs with extra fields and a superpotential. This means that we obtained two different GLSM proposals. Both of the proposals are U(1) gauge theories with a neutral chiral multiplet, two fundamental and two antifundamental chiral multiples, but one of the GLSMs also includes extra neutral fields that couple to the other fields through a superpotential (the $4 \mathrm{~d}-2 \mathrm{~d}$ maps of parameters are of-course different). At the level of the sphere partition function, the two GLSMs are equivalent.

\subsection{3 $S$-dual of the $c_{i}=1$ case}

Let us now move on to the strings that are $S$-dual to those of section 6.2.1. We will discuss these strings in detail in this subsection. We will include also the mesonic strings in our discussion. Thus, the general formula we derived for the two-sphere partition function, equation (5.12), which applies only for baryonic strings, is not sufficient for the analysis of this section.

When the U(1) flavor symmetry (6.10) is gauged, the four-ellipsoid partition function reads

$$
\begin{aligned}
Z_{S_{b}^{4}}= & \int d \hat{a} d \hat{a}^{\prime} e^{-\frac{16 \pi^{2}}{g^{2}} \hat{a}^{2}} e^{-\frac{16 \pi^{2}}{e^{2}} \hat{a}^{\prime 2}} e^{32 i \pi^{2} \hat{\xi} \hat{a}^{\prime}} \Upsilon_{b}(2 i \hat{a}) \Upsilon_{b}(-2 i \hat{a}) \\
& \left(\Upsilon_{b}\left(i\left(\hat{a}-2 \hat{a}^{\prime}\right)-i \hat{\mu}_{1}+\frac{Q}{2}\right) \Upsilon_{b}\left(-i\left(\hat{a}+2 \hat{a}^{\prime}\right)-i \hat{\mu}_{1}+\frac{Q}{2}\right)\right)^{-1} \\
& \left(\prod_{j=2}^{4} \Upsilon_{b}\left(i \hat{a}-i \hat{\mu}_{j}+\frac{Q}{2}\right) \Upsilon_{b}\left(-i \hat{a}-i \hat{\mu}_{j}+\frac{Q}{2}\right)\right)^{-1}\left|Z_{\text {inst }}\right|^{2}
\end{aligned}
$$

As we explained in section 4.2 we use a cut-off $\hat{\Lambda}$ for the matrix integral, and neglect $\mathcal{O}\left(\hat{\Lambda} e^{-\frac{\hat{\Lambda}^{2}}{e^{2}}}\right)+\mathcal{O}\left(\hat{\Lambda} e^{-\frac{\hat{\Lambda}^{2}}{g^{2}}}\right)$ contributions. First we perform the integration over $\hat{a}^{\prime}$. The 
poles in the upper half $\hat{a}^{\prime}$-plane are located at

$$
2 \hat{a}^{\prime}= \pm \hat{a}-\hat{\mu}_{1}+i\left(\frac{Q}{2}+k_{1} b+k_{1}^{\prime} b^{-1}\right), \quad k_{1}, k_{1}^{\prime} \in \mathbb{N} .
$$

The integrand is symmetric under $a \rightarrow-a$ and therefore we can focus on the poles

$$
2 \hat{a}^{\prime}=+\hat{a}-\hat{\mu}_{1}+i\left(\frac{Q}{2}+k_{1} b+k_{1}^{\prime} b^{-1}\right), \quad k_{1}, k_{1}^{\prime} \in \mathbb{N},
$$

and multiply the end result by 2 . The residues of the poles in (6.26) contain a $e^{16 \pi^{2} i \hat{\xi} \hat{a}}$ factor. Therefore, we need to close the contour of the $\hat{a}$ integrals from above. The contour will encircle the poles

$$
\hat{a}= \pm \hat{\mu}_{j}+i\left(\frac{Q}{2}+k_{2} b+k_{2}^{\prime} b^{-1}\right), \quad k_{2}, k_{2}^{\prime} \in \mathbb{N}, j=2,3,4 .
$$

The Cauchy integrations therefore result in a sum

$2(2 \pi i)^{2} e^{-16 \pi^{2} \hat{\xi} Q} \sum_{j=2}^{4}\left(e^{-16 i \pi^{2} \hat{\xi}\left(\hat{\mu}_{1}-\hat{\mu}_{j}\right)} Z_{\text {vac }, j}^{+} \sum_{K} e^{-16 \pi^{2} \hat{\xi} K b} Z_{K, j}^{+}+e^{-16 i \pi^{2} \hat{\xi}\left(\hat{\mu}_{1}+\hat{\mu}_{j}\right)} Z_{\text {vac }, j}^{-} \sum_{K} e^{-16 \pi^{2} \hat{\xi} K b} Z_{K, j}^{-}\right)+\ldots$

where the dots stand for contributions with $k_{1}^{\prime}+k_{2}^{\prime}>0 . Z_{\mathrm{vac}, j}^{ \pm} e^{-16 \pi^{2} \hat{\xi} K b} Z_{K, j}^{ \pm}$is the sum of the residues of the integrand in the poles

$$
\begin{aligned}
2 \hat{a}^{\prime} & =+\hat{a}-\hat{\mu}_{1}+i\left(\frac{Q}{2}+n b\right), \quad n=0,1,2, \ldots, K, \\
\hat{a} & = \pm \hat{\mu}_{j}+i\left(\frac{Q}{2}+(K-n) b\right) .
\end{aligned}
$$

The $Z^{-}$contributions correspond to the baryonic vacua and strings and the $Z^{+}$contributions correspond to the mesonic vacua and strings.

Evaluating the residue of the $K=0$ poles we find ${ }^{22}$

$$
\begin{aligned}
Z_{\mathrm{vac}, j=2}^{ \pm}= & e^{-\frac{4 \pi^{2}}{g^{2}}\left(\mp 2 \hat{\mu}_{2}-i Q\right)^{2}} e^{-\frac{4 \pi^{2}}{e^{2}}\left(-\left(\hat{\mu}_{1} \mp \hat{\mu}_{2}\right)+i Q\right)^{2}}\left(\Upsilon_{b}^{\prime}(0)^{-1}\right)^{2}\left(\prod_{k=3,4} \Upsilon_{b}\left(i\left( \pm \hat{\mu}_{2}+\hat{\mu}_{k}\right)\right)\right)^{-1} \\
& \left(\prod_{k=3,4} \Upsilon_{b}\left(i\left( \pm \hat{\mu}_{2}-\hat{\mu}_{k}\right)\right)\right)^{-1} Z_{\text {inst }}^{ \pm}(K=0, n=0, q) Z_{\text {inst }}^{ \pm}(K=0, n=0, \bar{q}), \quad
\end{aligned}
$$

where $Z_{\text {inst }}^{ \pm}(K, n, q)$ stands for the instanton partition function in the $\Omega$-background evaluated at

$$
\begin{aligned}
2 \hat{a}^{\prime} & =-\left(\hat{\mu}_{1} \mp \hat{\mu}_{2}\right)+i(Q+K b), \\
\hat{a} & = \pm \hat{\mu}_{2}+i\left(\frac{Q}{2}+(K-n) b\right) .
\end{aligned}
$$

\footnotetext{
${ }^{22}$ We use the symmetry between $\mu_{2}, \mu_{3}, \mu_{4}$ and write the expressions for $j=2$.
} 
The $S$-transformation rule (6.7) implies that the six strings we studied in section 6.2.1 should map to the strings described by $Z_{K, j}^{ \pm}$in the following way:

$$
\begin{aligned}
Z_{K,(1, j)}^{B}(q) & \rightarrow Z_{K, j}^{-}(1-q), \quad j=2,3,4, \\
Z_{K,(3,4)}^{B}(q) & \rightarrow Z_{K, 2}^{+}(1-q), \\
Z_{K,(2,4)}^{B}(q) & \rightarrow Z_{K, 3}^{+}(1-q), \\
Z_{K,(2,3)}^{B}(q) & \rightarrow Z_{K, 4}^{+}(1-q) .
\end{aligned}
$$

To find the two-sphere partition function for the minimal strings, we evaluate the sum of the residues of the $K=1$ poles. After using the shift identity (4.18) to separate the vacuum contributions we obtain

$$
\begin{aligned}
Z_{K=1, j=2}^{-}= & b^{4 i b \hat{\mu}_{2}+6 b^{2}+4} \gamma\left(-b^{2}\right) e^{\frac{4 \pi^{2}}{e^{2}}\left(2 i b\left(\hat{\mu}_{1}+\hat{\mu}_{2}\right)+3 b^{2}+2\right)} \\
& \left((q \bar{q})^{\left(-1-2 b^{2}-2 i b \hat{\mu}_{2}\right)} \frac{\gamma\left(2 i b \hat{\mu}_{2}+2 b^{2}+1\right)}{\prod_{k=3,4} \gamma\left(i b\left(\hat{\mu}_{2}-\hat{\mu}_{k}\right)+b^{2}+1\right) \prod_{k=3,4} \gamma\left(i b\left(\hat{\mu}_{2}+\hat{\mu}_{k}\right)+b^{2}+1\right)}\right. \\
& \left.\quad \times Z_{\text {inst }, n=1}^{-}(q) Z_{\text {inst }, n=1}^{-}(\bar{q})+\gamma\left(-2 i b \hat{\mu}_{2}-2 b^{2}-1\right) Z_{\text {inst }, n=0}^{-}(q) Z_{\text {inst }, n=0}^{-}(\bar{q})\right)
\end{aligned}
$$

with

$$
\begin{aligned}
& Z_{\text {inst }, n=1}^{-}(q)=(1-q)^{-i b\left(\hat{\mu}_{3}+\hat{\mu}_{4}\right)}{ }_{2} F_{1}\left(i b\left(\hat{\mu}_{2}-\hat{\mu}_{3}\right)+b^{2}+1, i b\left(\hat{\mu}_{2}-\hat{\mu}_{4}\right)+b^{2}+1,2 i b \hat{\mu}_{2}+2 b^{2}+2 \mid q\right), \\
& Z_{\text {inst }, n=0}^{-}(q)=(1-q)^{-i b\left(\hat{\mu}_{3}+\hat{\mu}_{4}\right)}{ }_{2} F_{1}\left(-i b\left(\hat{\mu}_{2}+\hat{\mu}_{3}\right)-b^{2},-i b\left(\hat{\mu}_{2}+\hat{\mu}_{4}\right)-b^{2},-2 i b \hat{\mu}_{2}-2 b^{2} \mid q\right) .
\end{aligned}
$$

For the instanton contributions we used the computation of appendix A and identity (B.3).

Our claim is that equation (6.36) is the two-sphere partition function of the GLSM obtained in section 6.2.1, with the dictionary between the four-dimensional and the twodimensional parameters given by the map

$$
\begin{aligned}
z & =1-q, \\
M_{1}-M_{2} & =b\left(\hat{\mu}_{3}+\hat{\mu}_{4}\right), \\
M_{1}+\tilde{M}_{1} & =b\left(\hat{\mu}_{2}+\hat{\mu}_{4}\right)-i b^{2}, \\
M_{1}+\tilde{M}_{2} & =b\left(\hat{\mu}_{2}+\hat{\mu}_{3}\right)-i b^{2}, \\
M_{X} & =-i b^{2},
\end{aligned}
$$

which is simply the composition of (6.18) with the S-transformation (6.7). Indeed, in appendix $\mathrm{C}$ we show that equation (6.19) can be written as

$$
\begin{aligned}
& Z_{S^{2}}=\gamma\left(-i M_{X}\right)(1-z)^{c-a-b}(1-\bar{z})^{c-a-b}(z \bar{z})^{\frac{c-1}{2}} 2 \pi(z \bar{z})^{-i\left(M_{1}+M_{2}\right) / 2} \times \\
& {\left[{ }_{2} F_{1}(a, b, a+b-c+1 \mid 1-z)_{2} F_{1}(a, b, a+b-c+1 \mid 1-\bar{z}) \frac{\gamma(c-a-b) \gamma(a) \gamma(b)}{\gamma(c-a) \gamma(c-b)}[(1-z)(1-\bar{z})]^{a+b-c}\right.} \\
& \left.+\gamma(a+b-c)_{2} F_{1}(c-a, c-b, c-a-b+1 \mid 1-z)_{2} F_{1}(c-a, c-b, c-a-b+1 \mid 1-\bar{z})\right]
\end{aligned}
$$


with

$$
\begin{aligned}
-i \tilde{M}_{1}-i M_{1} & =a, \\
-i \tilde{M}_{2}-i M_{1} & =b, \\
1+i\left(M_{2}-M_{1}\right) & =c .
\end{aligned}
$$

Under the map (6.38) expressions (6.36) and (6.39) match up to the multiplicative factor

$$
\begin{aligned}
& e^{\frac{4 \pi^{2}}{e^{2}}\left(2 i b\left(\hat{\mu}_{1}+\hat{\mu}_{2}\right)+3 b^{2}+2\right)}\left(b^{2}\right)^{i l\left(m_{1}+m_{2}+\tilde{m}_{1}+\tilde{m}_{2}\right)+3 b^{2}+2} \\
& \quad \times((1-z)(1-\bar{z}))^{-1-2 b^{2}-i l\left(m_{1}+m_{2}+\tilde{m}_{1}+\tilde{m}_{2}\right)}(z \bar{z})^{i M_{1}},
\end{aligned}
$$

which we interpret (as in the discussion below (4.29)) as an artifact of the regularization scheme.

To complete the check of the claims in (6.32)-(6.35) for the $K=1$ strings, we need to check that $Z_{K=1, j=2}^{+}$maps to (6.39) under the dictionary:

$$
\begin{aligned}
z & =1-q, \\
M_{1}-M_{2} & =b\left(\hat{\mu}_{3}-\hat{\mu}_{4}\right), \\
M_{1}+\tilde{M}_{1} & =-b\left(\hat{\mu}_{2}+\hat{\mu}_{4}\right)-i b^{2}, \\
M_{1}+\tilde{M}_{2} & =b\left(-\hat{\mu}_{2}+\hat{\mu}_{3}\right)-i b^{2}, \\
M_{X} & =-i b^{2},
\end{aligned}
$$

where $Z_{K=1, j=2}^{+}$is given by

$$
\begin{aligned}
Z_{K=1, j=2}^{+}= & b^{-4 i b \hat{\mu}_{2}+6 b^{2}+4} \gamma\left(-b^{2}\right) e^{\frac{4 \pi^{2}}{e^{2}}\left(2 i b\left(\hat{\mu}_{1}-\hat{\mu}_{2}\right)+3 b^{2}+2\right)} \\
& {\left[\gamma\left(2 i b \hat{\mu}_{2}-2 b^{2}-1\right) \frac{Z_{\text {inst }}^{+}(K=1, n=1, q) Z_{\text {inst }}^{+}(K=1, n=1, \bar{q})}{Z_{\text {inst }}^{+}(K=0, n=0, q) Z_{\text {inst }}^{+}(K=0, n=0, \bar{q})}+\right.} \\
& (q \bar{q})^{\left(-1-2 b^{2}+2 i b \hat{\mu}_{2}\right)} \frac{\gamma\left(-2 i b \hat{\mu}_{2}+2 b^{2}+1\right)}{\prod_{k=3,4} \gamma\left(i b\left(-\hat{\mu}_{2}-\hat{\mu}_{k}\right)+b^{2}+1\right) \prod_{k=3,4} \gamma\left(i b\left(-\hat{\mu}_{2}+\hat{\mu}_{k}\right)+b^{2}+1\right)} \\
& \left.\times \frac{Z_{\text {inst }}^{+}(K=1, n=0, q) Z_{\text {inst }}^{+}(K=1, n=0, \bar{q})}{Z_{\text {inst }}^{+}(K=0, n=0, q) Z_{\text {inst }}^{+}(K=0, n=0, \bar{q})}\right],
\end{aligned}
$$

Thus, we need to check that

$$
\begin{aligned}
& \frac{Z_{\text {inst }}^{+}(K=1, n=0, q)}{Z_{\text {inst }}^{+}(K=0, n=0, q)}=g(q, \hat{\mu})_{2} F_{1}\left(i b\left(\hat{\mu}_{2}+\hat{\mu}_{4}\right)-b^{2}, i b\left(\hat{\mu}_{2}-\hat{\mu}_{3}\right)-b^{2}, 2 i b \hat{\mu}_{2}-2 b^{2} \mid q\right), \\
& \frac{Z_{\text {inst }}^{+}(K=1, n=1, q)}{Z_{\text {inst }}^{+}(K=0, n=0, q)}=g(q, \hat{\mu})_{2} F_{1}\left(1-i b\left(\hat{\mu}_{2}+\hat{\mu}_{3}\right)+b^{2}, 1-i b\left(\hat{\mu}_{2}-\hat{\mu}_{4}\right)+b^{2}, 2-2 i b \hat{\mu}_{2}+2 b^{2} \mid q\right),
\end{aligned}
$$

with the same function $g(q, \mu)$. This is not a special case of the instanton analysis of appendix A, which applies only to the baryonic strings. We have checked that (6.44)(6.45) hold up to two instanton level. 


\subsubsection{Other transformations}

We discussed three out of the six triality elements. To complete the analysis, let us discuss the other three elements.

Each of the three $\mathrm{U}(1)$ generators we discussed is invariant under one of the triality transformations; $T_{B}, T_{T}$ and $T_{S}$ are invariant, respectively, under $S T S, S$ and $T$. This invariance manifests itself in the $\mathbb{Z}_{2}$ symmetry of the two-dimensional worldsheet theory

$$
t \rightarrow-t, m_{i} \leftrightarrow \tilde{m}_{i}
$$

where $m_{i}$ and $\tilde{m}_{i}$ are the twisted masses of the fundamental and anti-fundamental worldsheet chiral multiplets.

\section{Acknowledgments}

We would like to thank Shlomo Razamat and Talya Vaknin for fruitful discussions. We especially thank Jaume Gomis and Zohar Komargodski for collaboration throughout a large portion of this project and for many useful discussions. We also wish to thank an anonymous referee for valuable comments, corrections and suggestions. We thank the Perimeter Institute for Theoretical Physics and the Galileo Galilei Institute for Theoretical Physics for hospitality during the course of this project. This research was supported in part by Perimeter Institute for Theoretical Physics. Research at Perimeter Institute is supported by the Government of Canada through the Department of Innovation, Science and Economic Development and by the Province of Ontario through the Ministry of Research and Innovation. E.G. and A.K. are supported by the ERC STG grant 335182.

\section{A Non-perturbative contributions}

\section{A.1 Non-perturbative contributions to the four-ellipsoid partition function}

The non-perturbative contributions to equation (5.1) are given in terms of Nekrasov's instanton partition function [34], which in this case reads

$$
\begin{aligned}
& Z_{\text {inst }}=\sum_{\vec{Y}} q^{|\vec{Y}|} b^{\left(2 N_{c}-N_{f}\right)|\vec{Y}|} \prod_{a, b=1}^{N_{c}} Z_{a b}^{\mathrm{vec}}(\overrightarrow{\hat{a}}, \vec{Y}) \prod_{i=1}^{N_{f}} \prod_{a=1}^{N_{c}} Z_{a i}^{\mathrm{hyp}}(\overrightarrow{\hat{a}}, \overrightarrow{\hat{\mu}}, \vec{Y}), \\
& Z_{a b}^{\mathrm{vec}}=\prod_{r, s=1}^{\infty} \frac{\Gamma\left(Y_{a r}-Y_{b s}+b^{2}(r-s-1)+i b\left(w_{a} \cdot \hat{a}-w_{b} \cdot \hat{a}\right)\right)}{\Gamma\left(Y_{a r}-Y_{b s}+b^{2}(r-s)+i b\left(w_{a} \cdot \hat{a}-w_{b} \cdot \hat{a}\right)\right)} \frac{\Gamma\left(b^{2}(r-s)+i b\left(w_{a} \cdot \hat{a}-w_{b} \cdot \hat{a}\right)\right)}{\Gamma\left(b^{2}(r-s-1)+i b\left(w_{a} \cdot \hat{a}-w_{b} \cdot \hat{a}\right)\right)}, \\
& Z_{a i}^{\mathrm{hyp}}=\prod_{r=1}^{\infty} \frac{\Gamma\left(Y_{a r}+b^{2}(r-1)+b\left(i\left(w_{a} \cdot \hat{a}+c_{i} \hat{a}^{\prime}+\hat{\mu}_{i}\right)+\frac{Q}{2}\right)\right)}{\Gamma\left(b^{2}(r-1)+b\left(i\left(w_{a} \cdot \hat{a}+c_{i} \hat{a}^{\prime}+\hat{\mu}_{i}\right)+\frac{Q}{2}\right)\right)}
\end{aligned}
$$


where $q=e^{2 \pi i \tau_{s u\left(N_{c}\right)}{ }^{23}}$ The sum over $\vec{Y}$ is a sum over $N_{c}$-tuples of Young diagrams, $\vec{Y}=\left(Y_{1}, \ldots, Y_{N_{c}}\right), Y_{a r}$ is the height of the $r$ 'th column in the diagram $Y_{a}, Y_{a r} \geq Y_{a, r+1}$, and $|\vec{Y}|=\sum_{a=1}^{N_{c}} \sum_{r=1}^{\infty} Y_{a r}$.

We need to evaluate this for

$$
i w_{a} \cdot \hat{a}+i c_{a} \hat{a}^{\prime}+i \hat{\mu}_{a}+\frac{Q}{2}+k_{a} b=0, \quad a=1, \ldots, N_{c} .
$$

After a change of variables, this computation becomes a special case of computations that appeared in $[23,24,28]$. We repeat the computation here for the convenience of the reader. Substituting (A.2) into (A.1) we obtain

$$
\begin{aligned}
& Z_{\text {inst }}^{\text {res }}\left(\mathbb{M}_{i j},\left\{k_{a}\right\}, q\right)= \sum_{\left\{\vec{Y} \mid Y_{a, k_{a}+1}=0\right\}} q^{|\vec{Y}|} b^{\left(2 N_{c}-N_{f}\right)|\vec{Y}|} \prod_{i=1}^{N_{f}} \prod_{a=1}^{N_{c}} \prod_{r=1}^{k_{a}} \frac{\Gamma\left(Y_{a r}+b^{2}\left(r-1-k_{a}\right)+i b \mathbb{M}_{i a}\right)}{\Gamma\left(b^{2}\left(r-1-k_{a}\right)+i b \mathbb{M}_{i a}\right)} \\
& \prod_{a, b=1}^{N_{c}} \prod_{r=1}^{\infty} \prod_{s=1}^{\infty} \frac{\Gamma\left(Y_{a r}-Y_{b s}+b^{2}(r-s-1)+i b \mathbb{M}_{b a}-b^{2}\left(k_{a}-k_{b}\right)\right)}{\Gamma\left(Y_{a r}-Y_{b s}+b^{2}(r-s)+i b \mathbb{M}_{b a}-b^{2}\left(k_{a}-k_{b}\right)\right)} \\
& \times \frac{\Gamma\left(b^{2}(r-s)+i b \mathbb{M}_{b a}-b^{2}\left(k_{a}-k_{b}\right)\right)}{\Gamma\left(b^{2}(r-s-1)+i b \mathbb{M}_{b a}-b^{2}\left(k_{a}-k_{b}\right)\right)},
\end{aligned}
$$

where

$$
\mathbb{M}_{i a}=\hat{\mu}_{i}-\hat{\mu}_{a}+\Delta_{a i}\left(\sum_{b=1}^{N_{c}} \hat{\mu}_{b}-i N_{c} \frac{Q}{2}-i K b\right),
$$

and we used the fact that diagrams for which $Y_{a, k_{a}+1}>0$ cannot contribute since the hypermultiplet contribution for $r=k_{a}+1$ and $i=a$ multiplies everything by 0 . Simplifying this expression we get

$$
\begin{aligned}
& Z_{\text {inst }}^{\text {res }}\left(\mathbb{M}_{i j},\left\{k_{a}\right\}, q\right)= \\
& \quad \sum_{\left\{\vec{Y} \mid Y_{a, k_{a}+1}=0\right\}} q^{|\vec{Y}|} b^{\left(2 N_{c}-N_{f}\right)|\vec{Y}|}(-1)^{N_{c}|\vec{Y}|} \prod_{i=N_{c}+1}^{N_{f}} \prod_{a=1}^{N_{c}} \prod_{r=0}^{k_{a}-1}\left(-b^{2}(r+1)+i b \mathbb{M}_{i a}\right)_{Y_{a, k_{a}-r}} \\
& \quad \times \prod_{a, b=1}^{N_{c}} \prod_{r=0}^{k_{a}-1} \prod_{s=0}^{k_{b}-1} \frac{1}{\left(1-Y_{b, k_{b}-s}+Y_{a, k_{a}-r}+b^{2}(s-r)+i b \mathbb{M}_{b a}\right)_{Y_{b, k_{b}-s}-Y_{b, k_{b}-s+1}}} \\
& \quad \times \prod_{a, b=1}^{N_{c}} \prod_{r=0}^{k_{a}-1} \frac{\left(1+b^{2}\left(k_{b}-r\right)+i b \mathbb{M}_{b a}+Y_{a, k_{a}-r}-Y_{b, 1}\right)_{Y_{b, 1}}}{\left(1+b^{2}\left(k_{b}-r\right)+i b \mathbb{M}_{b a}\right)_{Y_{a, k_{a}-r}}}
\end{aligned}
$$

Here, $(x)_{n}$ is the Pochhammer symbol, defined as

$$
(x)_{n}=x(x+1)(x+2) \ldots(x+n-1)=\frac{\Gamma(x+n)}{\Gamma(x)} .
$$

\footnotetext{
${ }^{23}$ We ignore subtleties in the instanton partition function related to $\mathrm{U}(1)$ factors. These subtleties are expected to affect our results only through the overall factors multiplying the two-sphere partition functions that we identify as regularization ambiguities.
} 


\section{A.2 Non-perturbative contributions to the two-sphere partition function}

The two-sphere partition function of an $\mathcal{N}=(2,2)$ supersymmetric theory with gauge group $\mathrm{U}(K), N_{c}$ chiral multiplets in the fundamental representation, $N_{f}-N_{c}$ chiral multiplets in the anti-fundamental representation and one chiral multiplet in the adjoint representation, is given by equation (4.23), where the function $Z_{\left\{k_{a}\right\}}^{\text {vort }}$ is given by

$$
\begin{aligned}
& Z_{\left\{k_{a}\right\}}^{\mathrm{vort}}(z)= \\
& \sum_{\tilde{Y}}\left[(-1)^{N_{c}} z\right]^{\sum_{a=1}^{N_{c}} \sum_{r=0}^{k_{a}-1} \tilde{Y}_{a, k_{a}-r}} \\
& \times \prod_{a=1}^{N_{c}} \prod_{r=0}^{k_{a}-1} \frac{\prod_{j=1}^{N_{f}-N_{c}}\left(-i \tilde{M}_{j}-i M_{a}-i r M_{X}\right)_{\tilde{Y}_{a, k_{a}-r}}}{\prod_{b=1}^{N_{c}}\left(1+i M_{b}-i M_{a}+\left(k_{b}-r\right) i M_{X}\right)_{\tilde{Y}_{a, k_{a}-r}}} \\
& \times \prod_{a=1}^{N_{c}} \prod_{r=0}^{k_{a}-1} \frac{\prod_{b=1}^{N_{c}}\left(1+i M_{b}-i M_{a}+\left(k_{b}-r\right) i M_{X}+\tilde{Y}_{a, k_{a}-r}-\tilde{Y}_{b, 1}\right)_{\tilde{Y}_{b, 1}}}{\prod_{b=1}^{N_{c}} \prod_{s=0}^{k_{b}-1}\left(1+i M_{b}-i M_{a}+(s-r) i M_{X}+\tilde{Y}_{a, k_{a}-r}-\tilde{Y}_{b, k_{b}-s}\right)_{\tilde{Y}_{b, k_{b}-s}-\tilde{Y}_{b, k_{b}-s+1}}} .
\end{aligned}
$$

The sum over $\tilde{Y}$ is a sum over functions $\tilde{Y}:\left\{1, \ldots, N_{c}\right\} \times\left\{0, \ldots, k_{a}-1\right\} \rightarrow \mathbb{N}$, such that $\tilde{Y}_{a, k_{a}-r} \in \mathbb{N}$, where $a=1, \ldots, N_{c}, r=0, \ldots, k_{a}-1$, satisfies $\tilde{Y}_{a, k_{a}-r} \geq \tilde{Y}_{a, k_{a}-r+1}$. As before $z=e^{-2 \pi \xi_{2 d}+i \theta_{2 d}}$, and $M_{a}, \tilde{M}_{j}$ and $M_{X}$ are the complexified twisted masses for the chiral multiplets transforming in the fundamental, anti-fundamental and adjoint representations respectively.

We therefore find that the map

$$
\begin{array}{rlrl}
z & =b^{2 N_{c}-N_{f}} q, \\
m_{a}-m_{b} & =\mu_{a}-\mu_{b}+\Delta_{b a} \sum_{c=1}^{N_{c}} \mu_{c}, & & \\
m_{a}+\tilde{m}_{j-N_{c}} & =\mu_{a}-\mu_{j}+\Delta_{j a} \sum_{c=1}^{N_{c}} \mu_{c}, \Delta_{a b} N_{c}\left(b^{2}+1\right)+2 \Delta_{a b} K b^{2}, \\
m_{X} & =0, & R_{a}+\tilde{R}_{j-N_{c}}=\Delta_{a j} N_{c}\left(b^{2}+1\right)+2 \Delta_{a j} K b^{2}-2 b^{2}, \\
R_{X} & =-2 b^{2},
\end{array}
$$

where $a, b=1, \ldots, N_{c}, j=N_{c}+1, \ldots, N_{f}$, maps $Z_{\text {inst }}^{\text {res }}\left(\mathbb{M}_{i j},\left\{k_{a}\right\}, q\right)$ to $Z_{\left\{k_{a}\right\}}^{\text {vort }}(z)$. This is true for any $\mathrm{U}(1)$ charge assignment. For the strings discussed in sections 4.2, 5.2.1 and 5.2.2, this completes the identification of the outputs of our prescription as the two-sphere partition functions of the proposed worldsheet theories. In other examples, the observation above does not imply that the parameters of the charged sector of the worldsheet theory are given in terms of the four-dimensional parameters as in (A.8). For example, see section 6.2.3 (and appendix C), where we discuss a case in which the weak coupling regime maps to the strong coupling regime of the worldsheet theory and the identification of the worldsheet theory requires writing the worldsheet two-sphere partition function as an expansion around $z=1$. 


\section{B Useful identities}

In section 6.2 and appendix $\mathrm{C}$ we use the following hypergeometric and Gamma-function identities,

$$
\begin{aligned}
{ }_{2} F_{1}(a, b, c \mid z)= & \frac{\Gamma(c) \Gamma(c-a-b)}{\Gamma(c-a) \Gamma(c-b)}{ }_{2} F_{1}(a, b, a+b-c+1 \mid 1-z)+ \\
& \frac{\Gamma(a+b-c) \Gamma(c)}{\Gamma(a) \Gamma(b)}{ }_{2} F_{1}(c-a, c-b, c-a-b+1 \mid 1-z)(1-z)^{c-a-b} \\
{ }_{2} F_{1}(a, b, c \mid z)= & (1-z)^{-a}{ }_{2} F_{1}\left(a, c-b, c \mid \frac{z}{z-1}\right) \\
{ }_{2} F_{1}(a, b, c \mid z)= & (1-z)^{c-a-b}{ }_{2} F_{1}(c-a, c-b, c \mid z) \\
\Gamma(x)^{2}= & \gamma(x) \frac{\pi}{\sin (\pi x)}
\end{aligned}
$$

In appendix $\mathrm{C}$ we also use the identities:

$$
\begin{gathered}
\frac{\gamma(a) \gamma(b)}{\gamma(c)}\left(\frac{\Gamma(c) \Gamma(c-a-b)}{\Gamma(c-a) \Gamma(c-b)}\right)^{2}+\left(\begin{array}{c}
a \rightarrow 1-c+a \\
b \rightarrow 1-c+b \\
c \rightarrow 2-c
\end{array}\right)=\frac{\gamma(c-a-b) \gamma(a) \gamma(b)}{\gamma(c-a) \gamma(c-b)}, \\
\frac{\gamma(a) \gamma(b)}{\gamma(c)}\left(\frac{\Gamma(a+b-c) \Gamma(c)}{\Gamma(a) \Gamma(b)}\right)^{2}+\left(\begin{array}{c}
a \rightarrow 1-c+a \\
b \rightarrow 1-c+b \\
c \rightarrow 2-c
\end{array}\right)=\gamma(a+b-c), \\
\frac{\gamma(a) \gamma(b)}{\gamma(c)} \frac{\Gamma(c) \Gamma(c-a-b)}{\Gamma(c-a) \Gamma(c-b)} \frac{\Gamma(a+b-c) \Gamma(c)}{\Gamma(a) \Gamma(b)}+\left(\begin{array}{c}
a \rightarrow 1-c+a \\
b \rightarrow 1-c+b \\
c \rightarrow 2-c
\end{array}\right)=0 .
\end{gathered}
$$

To derive (B.5) one can use equation (B.4) to get

$$
\begin{aligned}
& \frac{\gamma(a) \gamma(b)}{\gamma(c)}\left(\frac{\Gamma(c) \Gamma(c-a-b)}{\Gamma(c-a) \Gamma(c-b)}\right)^{2}+\left(\begin{array}{c}
a \rightarrow 1-c+a \\
b \rightarrow 1-c+b \\
c \rightarrow 2-c
\end{array}\right)= \\
& \frac{\gamma(c-a-b) \gamma(a) \gamma(b)}{\gamma(c-a) \gamma(c-b)}\left(\frac{\sin (\pi(c-a)) \sin (\pi(c-b))}{\sin (\pi c) \sin (\pi(c-a-b))}+\frac{\sin (\pi(1-a)) \sin (\pi(1-b))}{\sin (\pi(2-c)) \sin (\pi(c-a-b))}\right)= \\
& \frac{\gamma(c-a-b) \gamma(a) \gamma(b)}{\gamma(c-a) \gamma(c-b)},
\end{aligned}
$$

where we also used the fact that $\gamma(x) \gamma(1-x)=1$. In the second step we used standard trigonometric identities. The derivation of (B.6)-(B.7) is similar. 


\section{Derivation of equation (6.39)}

We start with the formula (6.19) for the two-sphere partition function. For simplicity we denote

$$
\begin{aligned}
-i \tilde{M}_{1}-i M_{1} & =a, \\
-i \tilde{M}_{2}-i M_{1} & =b, \\
1+i\left(M_{2}-M_{1}\right) & =c,
\end{aligned}
$$

such that equation (6.19) becomes

$$
\begin{aligned}
Z_{S^{2}}= & 2 \pi \gamma\left(-i M_{X}\right)(z \bar{z})^{-i\left(M_{1}+M_{2}\right) / 2} \\
& \times\left((z \bar{z})^{\frac{c-1}{2}} \frac{\gamma(a) \gamma(b)}{\gamma(c)}{ }_{2} F_{1}(a, b, c \mid z){ }_{2} F_{1}(a, b, c \mid \bar{z})+\left(\begin{array}{c}
a \rightarrow 1-c+a \\
b \rightarrow 1-c+b \\
c \rightarrow 2-c
\end{array}\right)\right) .
\end{aligned}
$$

Using identity (B.1) we can write $Z_{S^{2}}=2 \pi \gamma\left(-i M_{X}\right)(z \bar{z})^{-i\left(M_{1}+M_{2}\right) / 2}\left(I_{1}+I_{2}+I_{3}+I_{4}\right)$, with

$$
\begin{aligned}
& I_{1}=(z \bar{z})^{\frac{c-1}{2}} \frac{\gamma(a) \gamma(b)}{\gamma(c)}\left(\frac{\Gamma(c) \Gamma(c-a-b)}{\Gamma(c-a) \Gamma(c-b)}\right)^{2} \times \\
& { }_{2} F_{1}(a, b, a+b-c+1 \mid 1-z){ }_{2} F_{1}(a, b, a+b-c+1 \mid 1-\bar{z})+\left(\begin{array}{c}
a \rightarrow 1-c+a \\
b \rightarrow 1-c+b \\
c \rightarrow 2-c
\end{array}\right), \\
& I_{2}=(z \bar{z})^{\frac{c-1}{2}} \frac{\gamma(a) \gamma(b)}{\gamma(c)}\left(\frac{\Gamma(a+b-c) \Gamma(c)}{\Gamma(a) \Gamma(b)}\right)^{2}(1-z)^{c-a-b}(1-\bar{z})^{c-a-b} \times \\
& { }_{2} F_{1}(c-a, c-b, c-a-b+1 \mid 1-z){ }_{2} F_{1}(c-a, c-b, c-a-b+1 \mid 1-\bar{z})+\left(\begin{array}{c}
a \rightarrow 1-c+a \\
b \rightarrow 1-c+b \\
c \rightarrow 2-c
\end{array}\right), \\
& I_{3}=(z \bar{z})^{\frac{c-1}{2}} \frac{\gamma(a) \gamma(b)}{\gamma(c)} \frac{\Gamma(c) \Gamma(c-a-b)}{\Gamma(c-a) \Gamma(c-b)} \frac{\Gamma(a+b-c) \Gamma(c)}{\Gamma(a) \Gamma(b)} \times \\
& { }_{2} F_{1}(a, b, a+b-c+1 \mid 1-z){ }_{2} F_{1}(c-a, c-b, c-a-b+1 \mid 1-\bar{z})(1-\bar{z})^{c-a-b}+\left(\begin{array}{c}
a \rightarrow 1-c+a \\
b \rightarrow 1-c+b \\
c \rightarrow 2-c
\end{array}\right), \\
& I_{4}=(z \bar{z})^{\frac{c-1}{2}} \frac{\gamma(a) \gamma(b)}{\gamma(c)} \frac{\Gamma(c) \Gamma(c-a-b)}{\Gamma(c-a) \Gamma(c-b)} \frac{\Gamma(a+b-c) \Gamma(c)}{\Gamma(a) \Gamma(b)} \times \\
& { }_{2} F_{1}(a, b, a+b-c+1 \mid 1-\bar{z}){ }_{2} F_{1}(c-a, c-b, c-a-b+1 \mid 1-z)(1-z)^{c-a-b}+\left(\begin{array}{c}
a \rightarrow 1-c+a \\
b \rightarrow 1-c+b \\
c \rightarrow 2-c
\end{array}\right) .
\end{aligned}
$$


Identity (B.3) implies that $z^{\frac{c-1}{2}}{ }_{2} F_{1}(a, b, a+b-c+1 \mid 1-z)$ and $z^{\frac{c-1}{2}}{ }_{2} F_{1}(c-a, c-b, c-$ $a-b+1 \mid 1-z)$ are invariant under $\left(\begin{array}{c}a \rightarrow 1-c+a \\ b \rightarrow 1-c+b \\ c \rightarrow 2-c\end{array}\right)$. We can therefore write:

$$
\begin{aligned}
& I_{1}=(z \bar{z})^{\frac{c-1}{2}}{ }_{2} F_{1}(a, b, a+b-c+1 \mid 1-z)_{2} F_{1}(a, b, a+b-c+1 \mid 1-\bar{z}) \times \\
& \left(\frac{\gamma(a) \gamma(b)}{\gamma(c)}\left(\frac{\Gamma(c) \Gamma(c-a-b)}{\Gamma(c-a) \Gamma(c-b)}\right)^{2}+\left(\begin{array}{c}
a \rightarrow 1-c+a \\
b \rightarrow 1-c+b \\
c \rightarrow 2-c
\end{array}\right)\right) \\
& I_{2}=(z \bar{z})^{\frac{c-1}{2}}\left(\frac{\gamma(a) \gamma(b)}{\gamma(c)}\left(\frac{\Gamma(a+b-c) \Gamma(c)}{\Gamma(a) \Gamma(b)}\right)^{2}+\left(\begin{array}{c}
a \rightarrow 1-c+a \\
b \rightarrow 1-c+b \\
c \rightarrow 2-c
\end{array}\right)\right) \times \\
& { }_{2} F_{1}(c-a, c-b, c-a-b+1 \mid 1-z){ }_{2} F_{1}(c-a, c-b, c-a-b+1 \mid 1-\bar{z})(1-z)^{c-a-b}(1-\bar{z})^{c-a-b}, \\
& I_{3}=(z \bar{z})^{\frac{c-1}{2}}\left(\frac{\gamma(a) \gamma(b)}{\gamma(c)} \frac{\Gamma(c) \Gamma(c-a-b)}{\Gamma(c-a) \Gamma(c-b)} \frac{\Gamma(a+b-c) \Gamma(c)}{\Gamma(a) \Gamma(b)}+\left(\begin{array}{c}
a \rightarrow 1-c+a \\
b \rightarrow 1-c+b \\
c \rightarrow 2-c
\end{array}\right)\right) \times \\
& { }_{2} F_{1}(a, b, a+b-c+1 \mid 1-z){ }_{2} F_{1}(c-a, c-b, c-a-b+1 \mid 1-\bar{z})(1-\bar{z})^{c-a-b}, \\
& I_{4}=(z \bar{z})^{\frac{c-1}{2}}\left(\frac{\gamma(a) \gamma(b)}{\gamma(c)} \frac{\Gamma(c) \Gamma(c-a-b)}{\Gamma(c-a) \Gamma(c-b)} \frac{\Gamma(a+b-c) \Gamma(c)}{\Gamma(a) \Gamma(b)}+\left(\begin{array}{c}
a \rightarrow 1-c+a \\
b \rightarrow 1-c+b \\
c \rightarrow 2-c
\end{array}\right)\right) \times \\
& { }_{2} F_{1}(a, b, a+b-c+1 \mid 1-\bar{z}){ }_{2} F_{1}(c-a, c-b, c-a-b+1 \mid 1-z)(1-z)^{c-a-b} .
\end{aligned}
$$

Using identities (B.5)-(B.7) we can simplify these expressions, and write

$$
\begin{aligned}
I_{1}= & (z \bar{z})^{\frac{c-1}{2}} \frac{\gamma(c-a-b) \gamma(a) \gamma(b)}{\gamma(c-a) \gamma(c-b)}{ }_{2} F_{1}(a, b, a+b-c+1 \mid 1-z)_{2} F_{1}(a, b, a+b-c+1 \mid 1-\bar{z}), \\
I_{2}= & (z \bar{z})^{\frac{c-1}{2}} \gamma(a+b-c)(1-z)^{c-a-b}(1-\bar{z})^{c-a-b} \times \\
& { }_{2} F_{1}(c-a, c-b, c-a-b+1 \mid 1-z){ }_{2} F_{1}(c-a, c-b, c-a-b+1 \mid 1-\bar{z}), \\
I_{3}= & I_{4}=0 .
\end{aligned}
$$

We therefore finally get

$$
\begin{aligned}
& Z_{S^{2}}=2 \pi \gamma\left(-i M_{X}\right)(z \bar{z})^{-i\left(M_{1}+M_{2}\right) / 2}(1-z)^{c-a-b}(1-\bar{z})^{c-a-b}(z \bar{z})^{\frac{c-1}{2}} \times \\
& {\left[{ }_{2} F_{1}(a, b, a+b-c+1 \mid 1-z)_{2} F_{1}(a, b, a+b-c+1 \mid 1-\bar{z}) \frac{\gamma(c-a-b) \gamma(a) \gamma(b)}{\gamma(c-a) \gamma(c-b)}[(1-z)(1-\bar{z})]^{a+b-c}\right.} \\
& \left.+\gamma(a+b-c)_{2} F_{1}(c-a, c-b, c-a-b+1 \mid 1-z){ }_{2} F_{1}(c-a, c-b, c-a-b+1 \mid 1-\bar{z})\right] .
\end{aligned}
$$




\section{Consistency check}

In this appendix we restrict to $N_{c}=2$ and $N_{f}=4$, and to strings that satisfy condition (3.4). The STS transformation in $\mathbf{S}_{3}$ keeps this condition invariant. We will show that the worldsheet theories and the spectrum of the worldsheet fields we proposed in sections 3.2 and 5.2.1 are consistent with this transformation.

Note that the $S T S$ transformation can be used to map strings with $c_{1}=c_{2}$ to strings with $c_{1} \neq c_{2}$. This allows us to understand the moduli of the $c_{1} \neq c_{2}$ strings (in particular, the spectrum of off-diagonal moduli) in terms of the better understood moduli of the $c_{1}=c_{2}$ strings.

The STS transformation maps $q$ to $1 / q$, and acts on the masses and U(1) charges as

$$
\begin{aligned}
\mu_{i} & \rightarrow \frac{1}{2}\left(\mu_{1}+\mu_{2}+\mu_{3}+\mu_{4}\right)-\mu_{5-i}, \\
c_{i} & \rightarrow \frac{1}{2}\left(c_{1}+c_{2}+c_{3}+c_{4}\right)-c_{5-i} .
\end{aligned}
$$

This transformation exchanges $\mathbb{M}_{12} \leftrightarrow \mathbb{M}_{34}, \mathbb{M}_{13} \leftrightarrow \mathbb{M}_{24}$, and $\mathbb{M}_{14} \leftrightarrow \mathbb{M}_{23}$, where $\mathbb{M}_{i j}$ is defined in equation (5.13). According to equations (5.15)-(5.16), the effect of this transformation on the worldsheet theory is that $z$ is mapped to $1 / z$, the fundamental and antifundamental multiplets are interchanged, while the spectrum of the neutral and adjoint fields remains invariant. This agrees exactly with the expectation based on the duality, since $z \rightarrow 1 / z$ acts as charge conjugation on the worldsheet theory. Therefore, accompanied by exchanging the fundamental and anti-fundamental representations, it is a symmetry of the worldsheet theory.

Open Access. This article is distributed under the terms of the Creative Commons Attribution License (CC-BY 4.0), which permits any use, distribution and reproduction in any medium, provided the original author(s) and source are credited.

\section{References}

[1] E. Gerchkovitz and A. Karasik, Vortex-strings in $\mathcal{N}=2 S Q C D$ and bulk-string decoupling, JHEP 02 (2018) 091 [arXiv:1710.02203] [INSPIRE].

[2] N. Dorey, The BPS spectra of two-dimensional supersymmetric gauge theories with twisted mass terms, JHEP 11 (1998) 005 [hep-th/9806056] [INSPIRE].

[3] A. Hanany and D. Tong, Vortices, instantons and branes, JHEP 07 (2003) 037 [hep-th/0306150] [INSPIRE].

[4] R. Auzzi, S. Bolognesi, J. Evslin, K. Konishi and A. Yung, NonAbelian superconductors: Vortices and confinement in $N=2$ SQCD, Nucl. Phys. B 673 (2003) 187 [hep-th/0307287] [INSPIRE].

[5] M. Shifman and A. Yung, NonAbelian string junctions as confined monopoles, Phys. Rev. D 70 (2004) 045004 [hep-th/0403149] [INSPIRE].

[6] A. Hanany and D. Tong, Vortex strings and four-dimensional gauge dynamics, JHEP 04 (2004) 066 [hep-th/0403158] [INSPIRE]. 
[7] M. Shifman and A. Yung, Non-Abelian semilocal strings in $N=2$ supersymmetric $Q C D$, Phys. Rev. D 73 (2006) 125012 [hep-th/0603134] [INSPIRE].

[8] M. Eto, Y. Isozumi, M. Nitta, K. Ohashi and N. Sakai, Moduli space of non-Abelian vortices, Phys. Rev. Lett. 96 (2006) 161601 [hep-th/0511088] [INSPIRE].

[9] R. Auzzi, M. Shifman and A. Yung, Composite non-Abelian flux tubes in $N=2 S Q C D$, Phys. Rev. D 73 (2006) 105012 [Erratum ibid. D 76 (2007) 109901] [hep-th/0511150] [INSPIRE].

[10] M. Eto et al., Constructing non-Abelian vortices with arbitrary gauge groups, AIP Conf. Proc. 1078 (2009) 483 [INSPIRE].

[11] L. Ferretti, S.B. Gudnason and K. Konishi, Non-Abelian vortices and monopoles in $\mathrm{SO}(N)$ theories, Nucl. Phys. B 789 (2008) 84 [arXiv: 0706.3854] [InSPIRE].

[12] N. Dorey, T.J. Hollowood and D. Tong, The BPS spectra of gauge theories in two-dimensions and four-dimensions, JHEP 05 (1999) 006 [hep-th/9902134] [INSPIRE].

[13] M. Eto et al., On the moduli space of semilocal strings and lumps, Phys. Rev. D 76 (2007) 105002 [arXiv: 0704.2218] [INSPIRE].

[14] M. Eto et al., Non-Abelian Vortices of Higher Winding Numbers, Phys. Rev. D 74 (2006) 065021 [hep-th/0607070] [INSPIRE].

[15] M. Shifman and A. Yung, Supersymmetric Solitons and How They Help Us Understand Non-Abelian Gauge Theories, Rev. Mod. Phys. 79 (2007) 1139 [hep-th/0703267] [INSPIRE].

[16] D. Tong, TASI lectures on solitons: Instantons, monopoles, vortices and kinks, in Theoretical Advanced Study Institute in Elementary Particle Physics: Many Dimensions of String Theory (TASI 2005), Boulder, Colorado, June 5-July 1, 2005 (2005) [hep-th/0509216] [INSPIRE].

[17] D. Tong, Quantum Vortex Strings: A Review, Annals Phys. 324 (2009) 30 [arXiv:0809.5060] [INSPIRE].

[18] M. Eto, Y. Isozumi, M. Nitta, K. Ohashi and N. Sakai, Solitons in the Higgs phase: The Moduli matrix approach, J. Phys. A 39 (2006) R315 [hep-th/0602170] [INSPIRE].

[19] V. Pestun, Localization of gauge theory on a four-sphere and supersymmetric Wilson loops, Commun. Math. Phys. 313 (2012) 71 [arXiv:0712.2824] [inSPIRE].

[20] N. Hama and K. Hosomichi, Seiberg-Witten Theories on Ellipsoids, JHEP 09 (2012) 033 [arXiv: 1206.6359] [INSPIRE].

[21] N. Doroud, J. Gomis, B. Le Floch and S. Lee, Exact Results in D $=2$ Supersymmetric Gauge Theories, JHEP 05 (2013) 093 [arXiv:1206.2606] [INSPIRE].

[22] F. Benini and S. Cremonesi, Partition Functions of $\mathcal{N}=(2,2)$ Gauge Theories on $S^{2}$ and Vortices, Commun. Math. Phys. 334 (2015) 1483 [arXiv:1206.2356] [INSPIRE].

[23] H.-Y. Chen and T.-H. Tsai, On Higgs branch localization of Seiberg-Witten theories on an ellipsoid, PTEP 2016 (2016) 013B09 [arXiv: 1506.04390] [INSPIRE].

[24] Y. Pan and W. Peelaers, Ellipsoid partition function from Seiberg-Witten monopoles, JHEP 10 (2015) 183 [arXiv: 1508.07329] [INSPIRE].

[25] H.-Y. Chen, N. Dorey, T.J. Hollowood and S. Lee, A New 2d/4d Duality via Integrability, JHEP 09 (2011) 040 [arXiv: 1104.3021] [INSPIRE].

[26] T. Fujimori, T. Kimura, M. Nitta and K. Ohashi, $2 d$ partition function in $\Omega$-background and vortex/instanton correspondence, JHEP 12 (2015) 110 [arXiv:1509.08630] [INSPIRE]. 
[27] J. Gomis, B. Le Floch, Y. Pan and W. Peelaers, Intersecting Surface Defects and Two-Dimensional CFT, Phys. Rev. D 96 (2017) 045003 [arXiv:1610.03501] [InSPIRE].

[28] Y. Pan and W. Peelaers, Intersecting Surface Defects and Instanton Partition Functions, JHEP 07 (2017) 073 [arXiv: 1612.04839] [INSPIRE].

[29] D. Gaiotto, L. Rastelli and S.S. Razamat, Bootstrapping the superconformal index with surface defects, JHEP 01 (2013) 022 [arXiv:1207.3577] [INSPIRE].

[30] N. Seiberg and E. Witten, Monopoles, duality and chiral symmetry breaking in $N=2$ supersymmetric QCD, Nucl. Phys. B 431 (1994) 484 [hep-th/9408099] [InSPIRE].

[31] N.S. Manton and J.M. Speight, Asymptotic interactions of critically coupled vortices, Commun. Math. Phys. 236 (2003) 535 [hep-th/0205307] [INSPIRE].

[32] C.H. Taubes, Arbitrary N: Vortex Solutions to the First Order Landau-Ginzburg Equations, Commun. Math. Phys. 72 (1980) 277 [InSPIRE].

[33] D. Tong, Monopoles in the Higgs phase, Phys. Rev. D 69 (2004) 065003 [hep-th/0307302] [INSPIRE].

[34] N.A. Nekrasov, Seiberg-Witten prepotential from instanton counting, Adv. Theor. Math. Phys. 7 (2003) 831 [hep-th/0206161] [INSPIRE].

[35] J. Gomis and S. Lee, Exact Kähler Potential from Gauge Theory and Mirror Symmetry, JHEP 04 (2013) 019 [arXiv: 1210.6022] [INSPIRE].

[36] J. Gomis and B. Le Floch, M2-brane surface operators and gauge theory dualities in Toda, JHEP 04 (2016) 183 [arXiv: 1407.1852] [INSPIRE].

[37] E. Gerchkovitz, J. Gomis and Z. Komargodski, Sphere Partition Functions and the Zamolodchikov Metric, JHEP 11 (2014) 001 [arXiv:1405.7271] [INSPIRE].

[38] D. Gaiotto, $N=2$ dualities, JHEP 08 (2012) 034 [arXiv:0904.2715] [INSPIRE].

[39] L.F. Alday, D. Gaiotto and Y. Tachikawa, Liouville Correlation Functions from Four-dimensional Gauge Theories, Lett. Math. Phys. 91 (2010) 167 [arXiv:0906.3219] [INSPIRE]. 